\title{
Étude de quelques caractéristiques de l'écoulement dans la partie aval des évacuateurs de surface
}

\author{
Study of some flow charecteristics \\ in the downstream part of spillways
}

\author{
PAR R. MAITRE ET S. OBOMENSKY
}

INGE

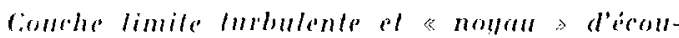

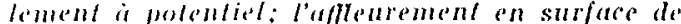
la courhe larbulente (anec émmlsion et gonflement de la neime se déplater de lomont bers

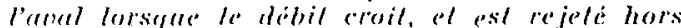
de l'ostorage pour une cerfaine bralear da firant d'ean pelatif. Bilan d'energie: la proportion d'energie dissipies sur le coursier diminue lorsque le dibit crobl. Mesures at Saint-Eliennecanlalis al Chaslang: nilesses molyennes

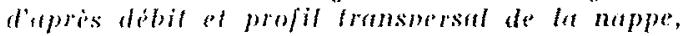
bifesses en surface al pris da fond, pressions sur le radiog resullals des mesures ef trates de la lighe de rharga . Comparaison des resul.

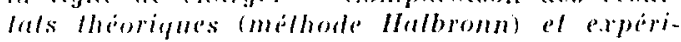

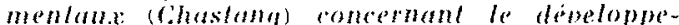
ment de la rourhe limite; palidité de la simililude. - Elude des perfes de rharge sur les fuacuateurs a partir des resultals experimentatur de Saint-kfienno-Cantales el de Chastang: ses conséquences sur la lenue des onbrages ef la dissipalion d'inergic.
\end{abstract}

\begin{abstract}
Turbulemt houmlary layer and " nucleus" of potential flow; the point at which the turbulent la!ger lenels off on the surface (with emmlsion and inflation of the streami momes from the upstream tementeds the domentstream utren the abchurge increases and is rejected ontside the strurlure for a eretain balut of the relatibe dranght. Balance of energy: hhe proportion of eneryll dissipated on the channel is less when the discherge increases. Measurements at st-

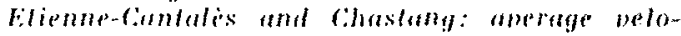
rilies arearding to discharge and lanswersal profile af the nappe, elecities on the surface and neat the bollam, pressures on the apron. kesulls of measuring and plolling the heal line. Comparison of theoretial (Mr. Malbromn) and experimenlal (Chastany) results concerning the denelopment of the boundary loyer; validity of the similitude. Sludy of head losses on the spillwall from experimental resulls oblained at St-Elienne-Cantales and Chastang: he effects on the slability of structures and energy dis. sipation.
\end{abstract}

\section{INTRODUCTION}

Cetle nole a pour objet, non de présenter des considérations theoriques originales, mais de rassembler de nombreuses observations éparses existantes, de présenter les résultats des mesures ricemment effectuces à Saint-Elienne-Cantales (c) à Chastang, de confronter ces résultats expérimentaux avec les methodes de calcul theoriques, en cherchant à dégager de ces progrès dans la connaissance des phénomènes des conclusions applicables aux ouvages futurs.

Le probleme de l'écoulement dans les coursiers des 6́ncuateurs de surface se pose sous deux aspects :
1" Aspect theorique de probleme.

a) Ios lois de lécoulement aux grandes vilesses :

Si les lois de l'écoulement et des pertes de charge sont diffirentes des lois chassiques, ha validité de l'étude en similitude de Frovos de ces ćcoulements peut être mise en question.

En effet, contrairement à ce qui se passe, en géneral, pour les eludes sur modiele, les nombres de Rrivolds des écoulements sur des évacualeurs en modele réduit sont tres largement suffisants pour que les écoulements réalisés soient 
pleinement turbulents (point figuratif dans la harpe de Nikuradse), Mais, sur l'ouvrage réel, on sort du domaine exploré à l'heure actuelle, le nombre de Reynolos atteignant $10^{7}$ ou $10^{s}$.

b) Les conditions de formation et les lois du développement de l'émulsion air-eau :

Ce problème très complexe et difficilement accessible par les moyens expérimentaux actucls ne sera pas abordé dans cette note, dont il déborde le cadre.

\section{$2^{n}$ Aspect pratique du problème.}

Ce sont les questions qui se posent depuis que l'on conçoit de tels ouvrages et qui sont étroitement liées aux précédentes :

- Fxistence d'une vitesse limite.
- Importance du gonflement éventuel de la veine sous l'efret de l'émulsion.

- Bilan de la dissipalion de l'énergie sur de tels ouvrages, importance relative de la dissipation par frottement sur l'ouvrage, par frottement dans l'air, par lourbillons of remous à l'aval, par érosion sur le fond.

Nous aborderons successivement ces deux aspects du probleme en nous attachant plus particulièrement à la question des lois de l'écoulcment d'une part et à celle du bilan d'énergie d'autre part.

Nous rappellerons tout d'abord l'aspect qualitatif des phénomènes, puis nous relaterons les mesures effectućes sur des ouvrages réels, mesures dont les résultats nous permetlront d'aborder l'aspect quantitatif de ces problemes.

\section{PREM I ER E PART I E}

\section{ANALYSE QUALITATIVE DES PHÉNOMENES}

\section{A) Nature de l'écoulement sur les évacuateurs de surface; son évolution en fonction du débit}

Lorsque, sur un ouvrage déversant donné supposé de largeur infinie, on évacue un faible débit, on observe (fig. 1) une courte zone d'écoulement à potentiel sur le seuil déversant, cependant que la couche limite turbulente se développe à partir du début du radier pour venir apparaître au point d'affleurement ou point critique C. A l'aval de ce point, l'écoulement est entièrement turbulent el si les vitesses (c'est-à-dire la hauteur de chute) sont suffisantes, on a une imulsion en surface.

Au contraire, si le débit évacué est beaucoup plus important (fig. 2), l'importance de la couche limite variant peu, le point d'affleurement se trouve rejeté à l'aval de l'ouvrage, si bien que l'écoulement se compose dans une section quclconque de l'ouvrage d'une couche limite turbulente et d'un « noyau » d'écoulement à potentiel, donc à énergie constante.

Le cas limite correspond à un point d'af-

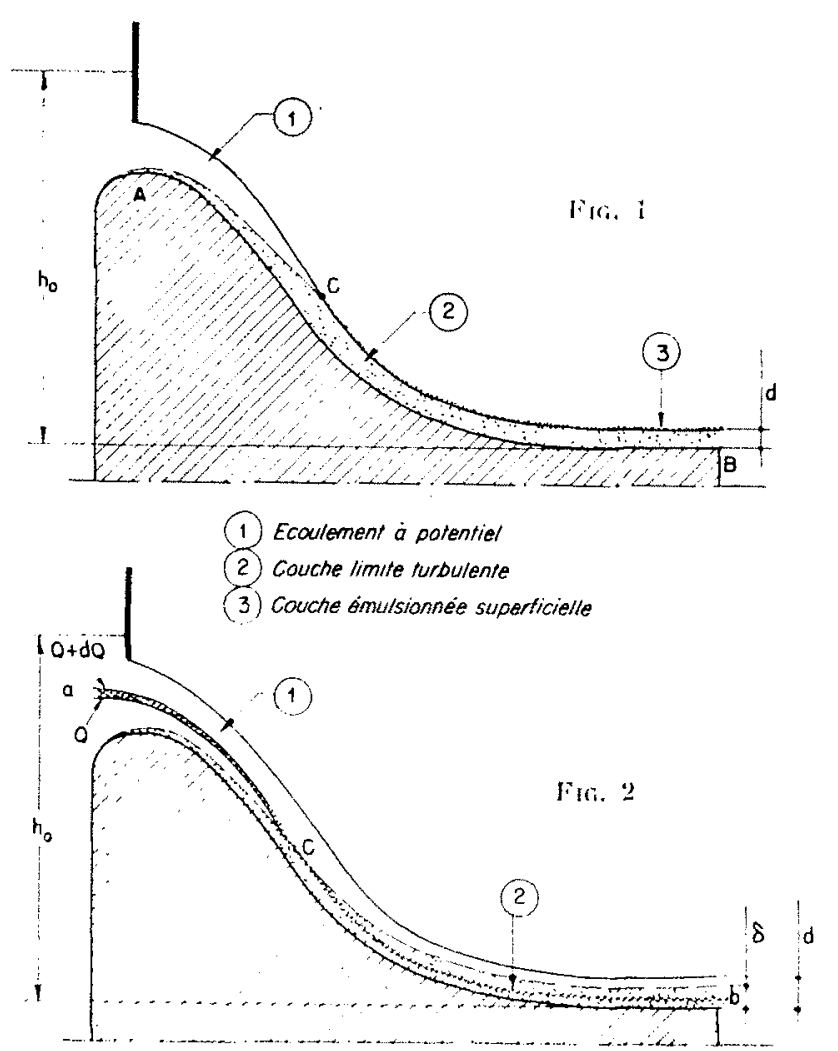




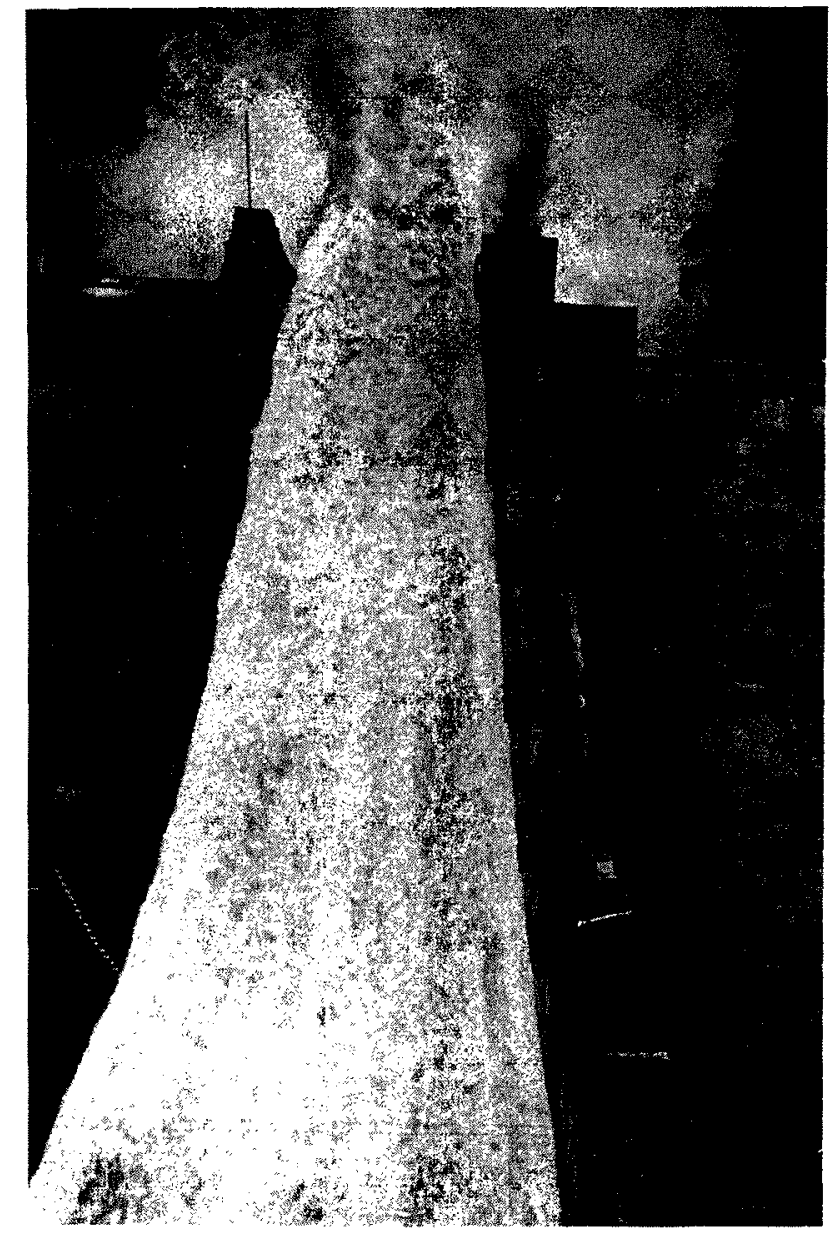

Fir. 3. - Débit non mesuré.

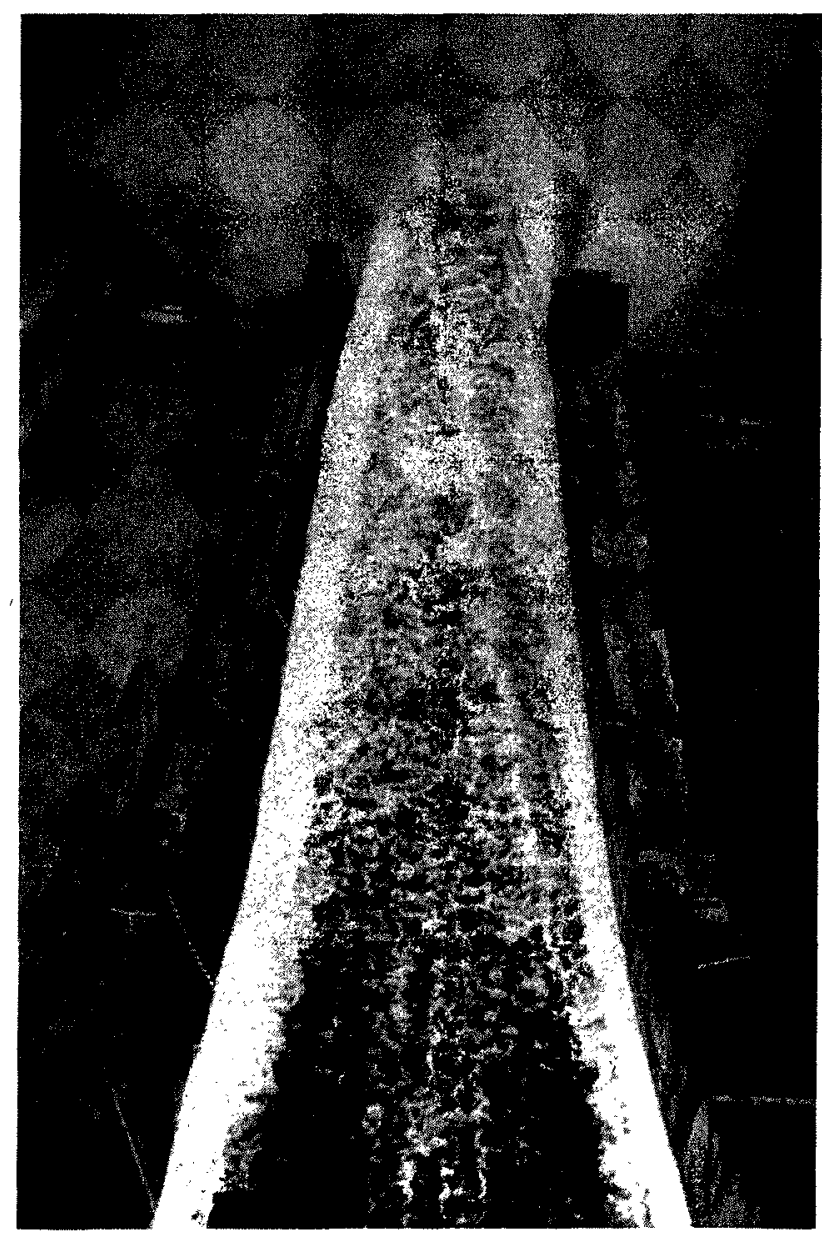

Fig. 4. - Débit non mesuré.
PI.ANCHE I

EVACATIEET DE

SANT-ETHENE-CANTALES.

VIT HE L'ECOU.IEMENT poln

WES WERTS CHOHSNANTS.

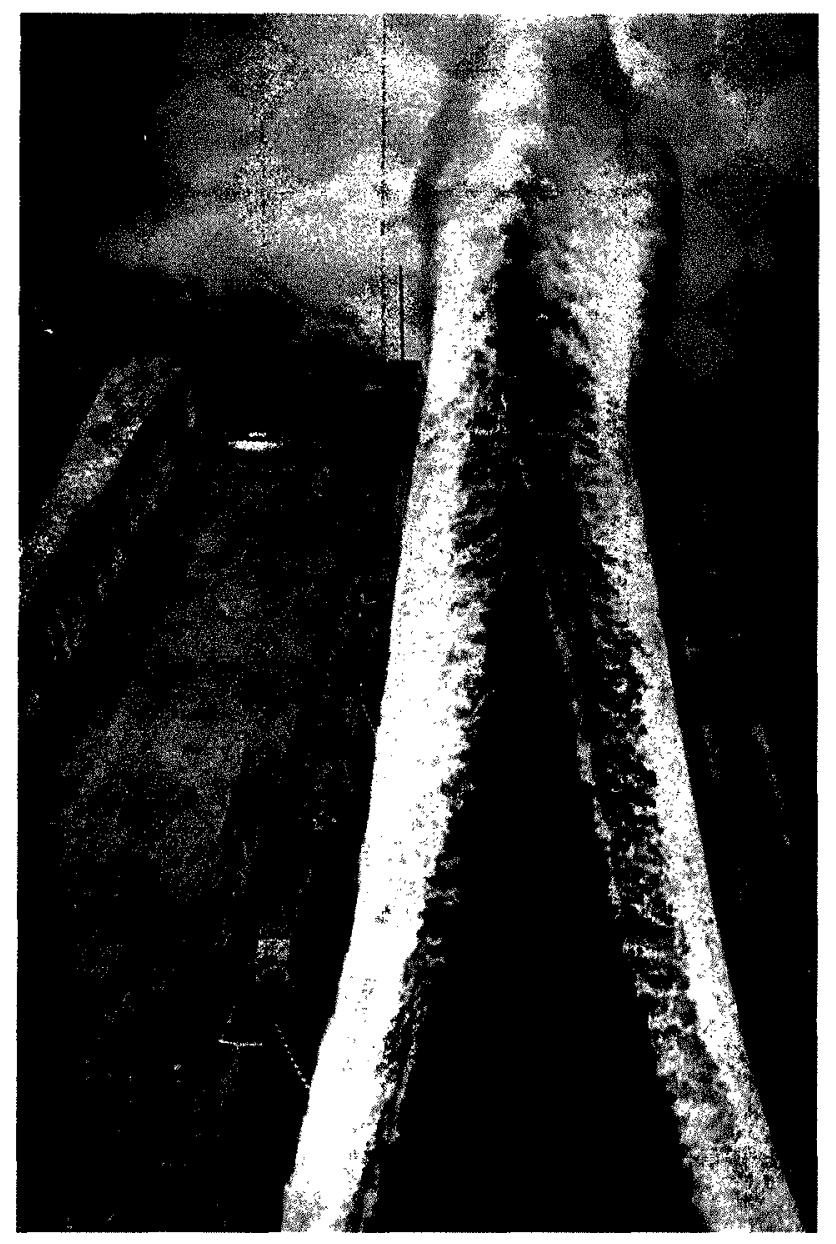

Fin. .. - $9-23013^{3 / 4}$

fleurement situe a l'extremite aval de l'ouvrage, ce qui a lieu pour un certain débit $Q=Q_{c}$.

Pour un onvage réd de largeur finie, ce schema se trouve modifie dans le cas de débits importants par la présence d'une couche limite laterale qui se developpe a partir des guideaux sur toute l'epaissenr de la veine liquide. Le noyau d'écoulement à polentiel se lrouve ainsi en contact sur trois eotés avec une couche limite turliulente.

Cette évolution de l'ecoulement en fonction du débit est générale, comme le montrent les pliotos 
PLANCHE II

Evaclatelin DF SaINT-ETHENe-Cantalis.

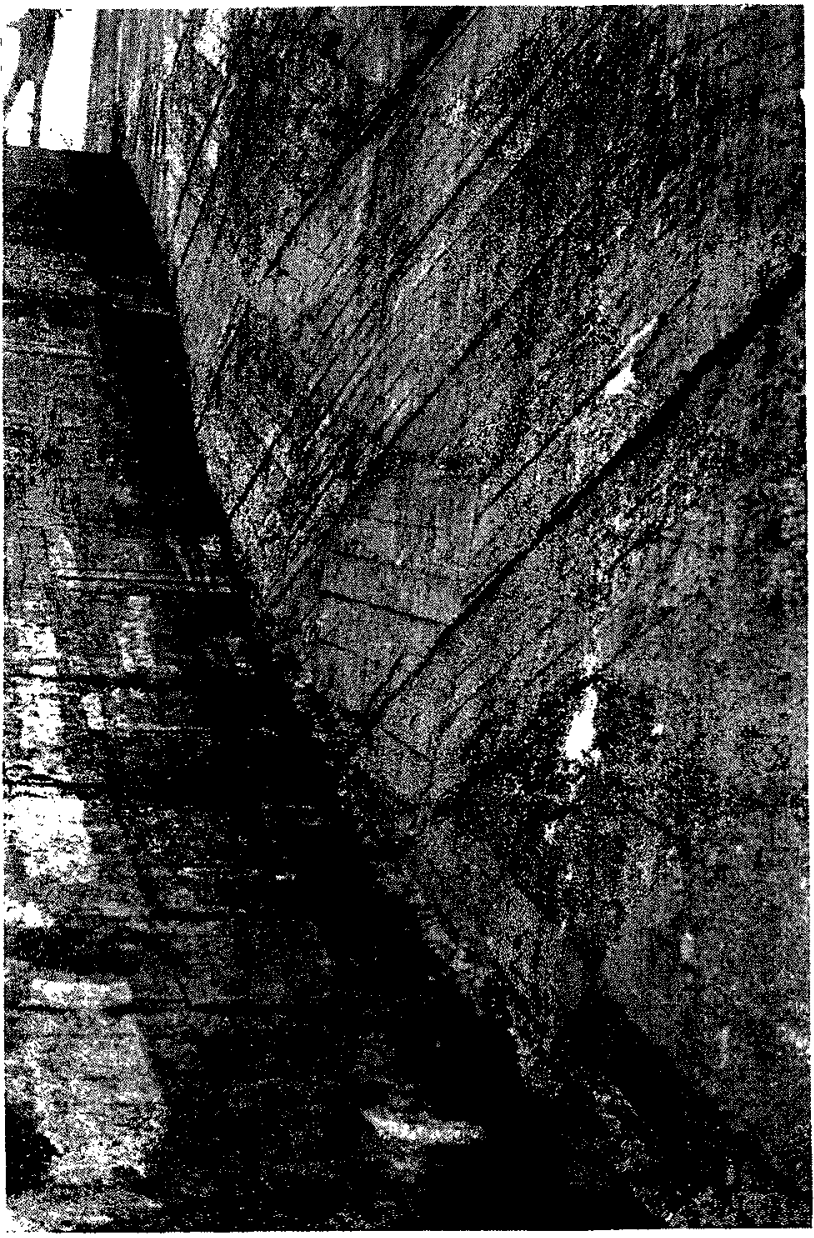

Fia, 6. - Aspeel des parois.

prises à Saint-Etienne-Cantales et à Chastang (planches I, II, III).

On remarque, à partir du schéma qualitatif précédent, que :

a) Sur un ouvrage donné, l'émulsion existe aux petits débits et disparaît aux débits importants. Il en est donc de même du gonflement de la veine qui en résulte. Ce gonflement de la veine ne pose donc pas de problème dans la pratique pour les ouvrages suffisamment courts: la hauteur des bajoyers ne doit tenir compte que du léger bourrelet émulsionné dù à la couche limite latérale (fig. 18). Il est à noter que l'imporlance plus grande de ce bourrelet sur l'évacuateur de Saint-Etienne-Cantalès provient de la convergence de l'ouvrage en plan : cette convergence provoque en effet la formation d'une veine réfléchie superficielle plus ou moins émulsion-

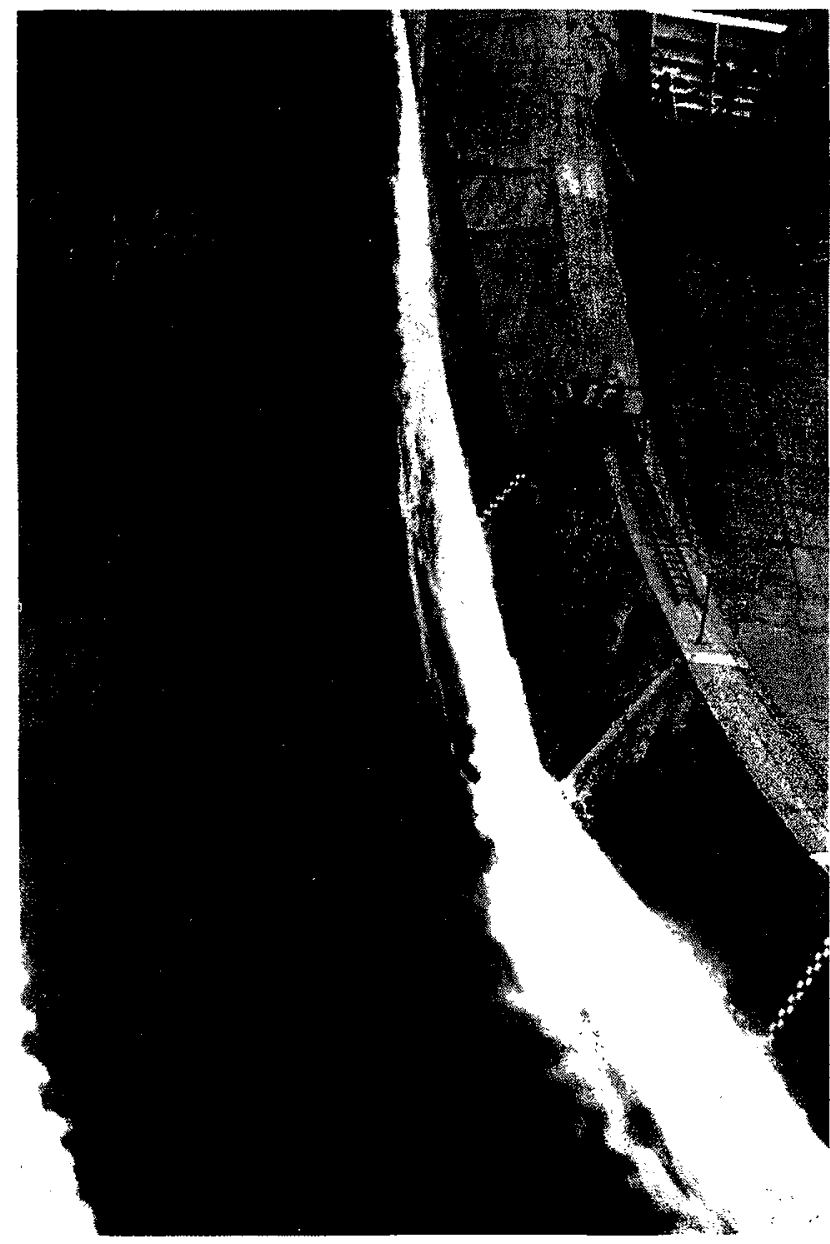

Fidi. $7 .-$ Ecoulement a u voisinage des parois $0=230 \mathrm{~m}^{3} / \mathrm{s}$.

née, mais aucunement lićc à la couche limite (fig. 5 et 17 ).

b) Il apparaît, indépendamment de toute théorie, que l'épaisseur de la couche iimite croit avec l'abscisse curviligne de A vers $B$. Si l'on considère seulement, en première approximation, la couche limite de fond, il est logique de faire apparaître la « Iongueur relative » de l'ouvrage $\mathrm{L} / d$, avec :

\section{$\mathrm{L}=$ = longueur développée du coursier; \\ $d=$ tirant d'eau à l'extrémité aval.}

Cette longueur relative varie avec le débit pour un ouvrage donné. Si L/d est grand, ce qui correspond soit à un ouvrage Jong, soit à un faible débit, le point d'affleurement $\mathrm{C}$ se trouve sur l'ouvrage (fig. 1). Si L/d est faible (cas d'un ouvrage court ou d'un fort débit), le point d'al'fleurement se trouve rejeté à l'aval de l'ourrage. 
Il $\mathrm{y}$ a d'autres paramètres qui interviennent dans le développement de la couche limite : rugosité du radier, hauteur de l'ouvrage, etc...

Il est toutefois intéressant de comparer, à titre indicatif, le domaine de variation du paramètre $\mathrm{L} / d$ en fonction du débit pour quelques ouvages existants, compte tenu du débit maximum de chacun d'eux.

Pour des raisons de simplicité, le lirant d'eau d a été pris égal au tirant d'eau thérique $d_{n}$ eorrespondant à la vitesse théorique $\mathrm{V}_{n}=\sqrt{2} g \mathrm{H}$, $\mathrm{H}$ représentant la dénivelée entre le plan d'eau amont et la surface de la vine à l'extrémité aval de l'évacuateur.

Ceci n'a aucune importance, le paramètre $L / d$ aysunt surtout une valeur comparative. Les résultals de ces évaluations sont rassemblés dans le tableau ci-après et reportés sur le diagramme de la figure 8 en supposant que le tirant d'eat d ì l'extrímité aval croît proportionnellement au débit ivacué par l'ouvrage, approximation que justifie le caractere indicatif de ce diagramme.

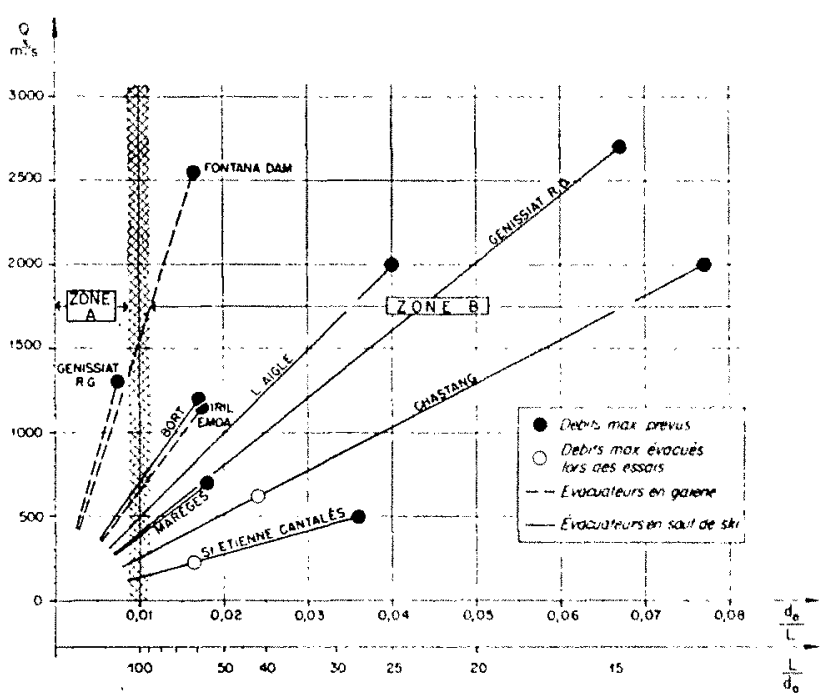

Fia. 8. - Diagramme comparalif des longueurs relatives de quelques cracuateurs. -.. \%one A. Eeoulement turbulent ens suface de la parlic aval: l'bulsion peut exister. - \%one B. Eowlement a polentiel en surface : l'émulsion ne peul pas exister.

CARACTHISTIQCES DE QCELQUES EVACATELRS EN SATT DE SHI

\begin{tabular}{|c|c|c|c|c|c|c|c|c|c|c|c|}
\hline & \multirow{2}{*}{$\begin{array}{l}\text { Chute } \\
\text { sur l'ou- } \\
\text { rrage } \\
h_{0} \mathrm{~m}\end{array}$} & \multirow{2}{*}{$\begin{array}{l}\text { Chute } \\
\text { totale } \\
\mathrm{H}_{0} \quad \mathrm{~m}\end{array}$} & \multirow{2}{*}{$h_{0} / \mathrm{H}_{0}$} & \multirow{2}{*}{$\begin{array}{l}\text { Long. } \\
\text { dévelop. } \\
\mathrm{I} \quad \mathrm{m}\end{array}$} & \multirow{2}{*}{$\begin{array}{c}\text { Débit } \\
\text { maxim. } \\
\text { unitaire } \\
\mathrm{m}^{3} / \mathrm{s}\end{array}$} & \multicolumn{2}{|c|}{$\begin{array}{l}\text { Largeur } \\
\text { du coursier } \\
\text { mI }\end{array}$} & \multirow{2}{*}{$\begin{array}{c}\text { Debit } \\
\text { maxim. } \\
\text { parm. } \\
\text { de large } \\
\text { in at } \\
\text { sortie } \\
q \mathrm{~m}^{*} / \mathrm{s} \\
\end{array}$} & \multirow{2}{*}{$\begin{array}{c}\text { Vitesse } \\
\text { théorique } \\
\text { i la sortic } \\
V_{0} \mathrm{~m} / \mathrm{s}\end{array}$} & \multirow{2}{*}{$\begin{array}{l}\text { Tirant } \\
\text { deau } \\
\text { dhéo- } \\
\text { rique } \\
\text { iq la } \\
\text { sortic } \\
\text { dor me } \\
\end{array}$} & \multirow{2}{*}{$\begin{array}{l}\text { Longueur } \\
\text { relative } \\
\text { an débit } \\
\text { maxim. } \\
\text { L/d d }\end{array}$} \\
\hline & & & & & & $\stackrel{\grave{a}}{\text { lamont }}$ & $\begin{array}{c}a \\
\text { l'avial }\end{array}$ & & & & \\
\hline Marèges. & 37 & 77 & 0,48 & 55 & 700 & 26 & 26 & 27 & 27 & 1 & 55 \\
\hline St-Etionne-Cantal & 37 & 70 & 0,53 & 75 & 500 & 11 & 7 & 71 & 26,8 & 2,65 & 28 \\
\hline LAigle & 54 & 90 & 0,60 & 95 & 2000 & 25 & 16 & 125 & 32,55 & 3,85 & 25 \\
\hline Chastans & 42 & 71 & 0,59 & 68 & 2000 & 13,50 & 13,50 & 147 & 28.7 & 5,1 & 13 \\
\hline Bort. & 74 & 115 & 0,64 & 147 & 1200 & 18 & 13,15 & 91 & 38,1 & 2,5 & 59 \\
\hline Génissiat (R.D.)...... & 48 & 64 & 0,75 & 90 & 27100 & 18 & 15 & 180 & 29.1 & 6 & 15 \\
\hline
\end{tabular}

\section{B) Schéma de l'évolution du bilan d'énergie en fonction du débit évacué}

On peut déduire de la nalure de l'écoulement analysée ci-dessus un certain nombre d'indicalions qualitatives sur limportance relative des dirrérentes formes de dissipation dénergie el ses variations en fonction du débit.

Supposons, en reprenant le schéma de la figure 2, que l'écoulement sur un ouvrage déversant soit constitué par la superposilion d'une infinite de filels liquides élémentaires évacuant chacun le débit $d Q$, les lignes de courant représentant les lignes d'eau successives lorsque le débit croît. Il est possible de graduer les lignes de courant en notant "ligne de courant $Q$ » celle qui correspond à la ligne d'eau relative au passage du débit $Q$.
Considerons le diled liquide silua entre les lignes de eourant $Q$ el $Q+d Q$ of reaminons la variation de la charge :

$$
\mathrm{H}=\mathrm{Z}+\frac{\rho}{\pi}+\frac{y z}{2 !}
$$

le long de ce filet liquide.

Sur le parcours ac, le lilel s'icoule dans le noyau, en : $\mathrm{H}=\mathrm{C}^{\mathrm{a}}$. Sur le parcours $a b$, il se produit dans la couche lurbulente une perte de charge $\Delta H$ proportionnelle, toutes choses égales d'ailleurs, au trajet $c b$. Celle perte de charge varie done d'un filet liquide à l'autre du fait du déplacement du point $c$. 


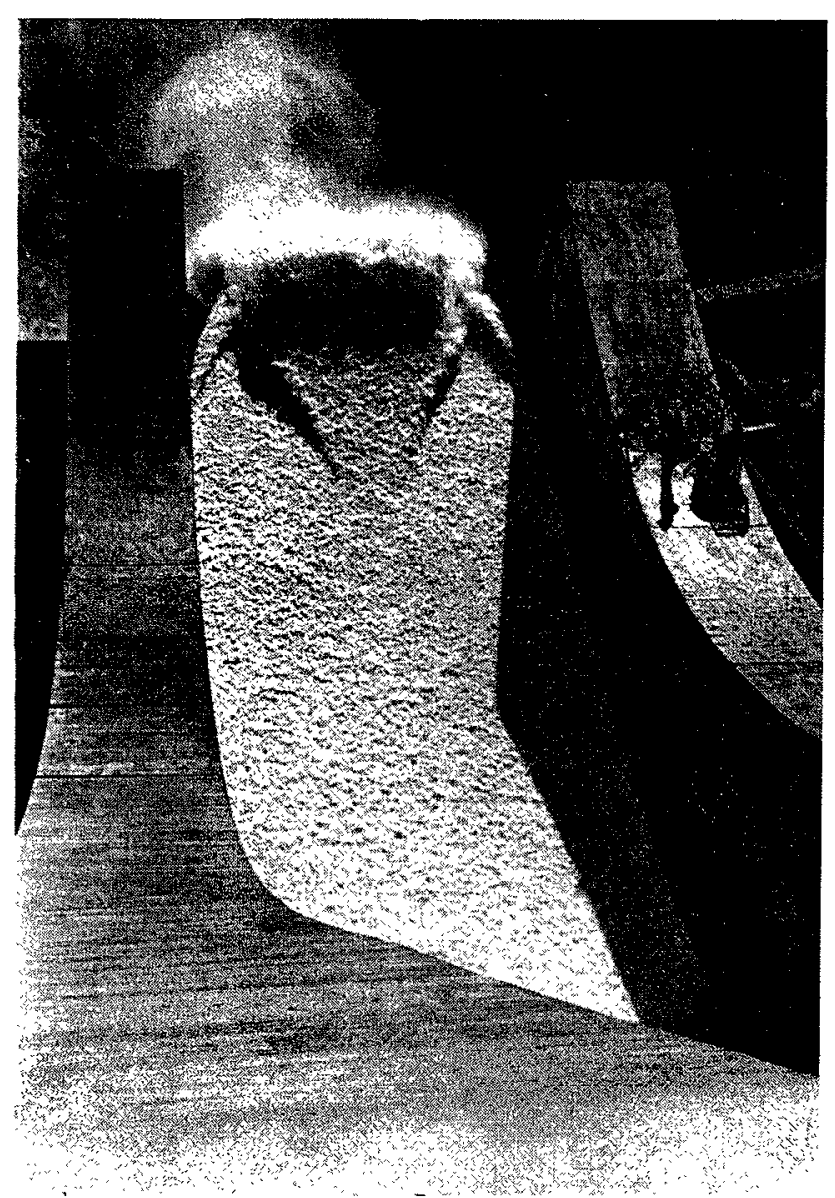

Fig. 9. - Ouverture de la vanne.

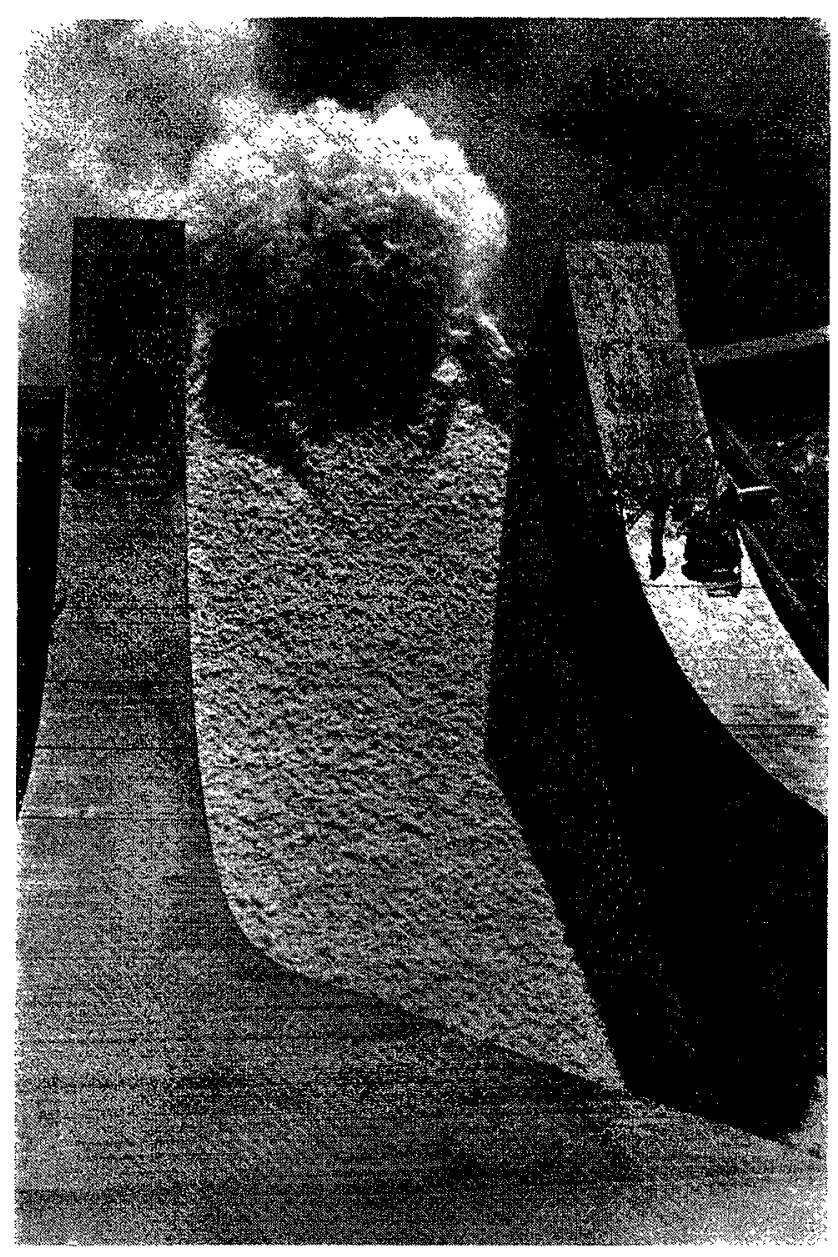

Fig. 10. - Débit non mesuré.

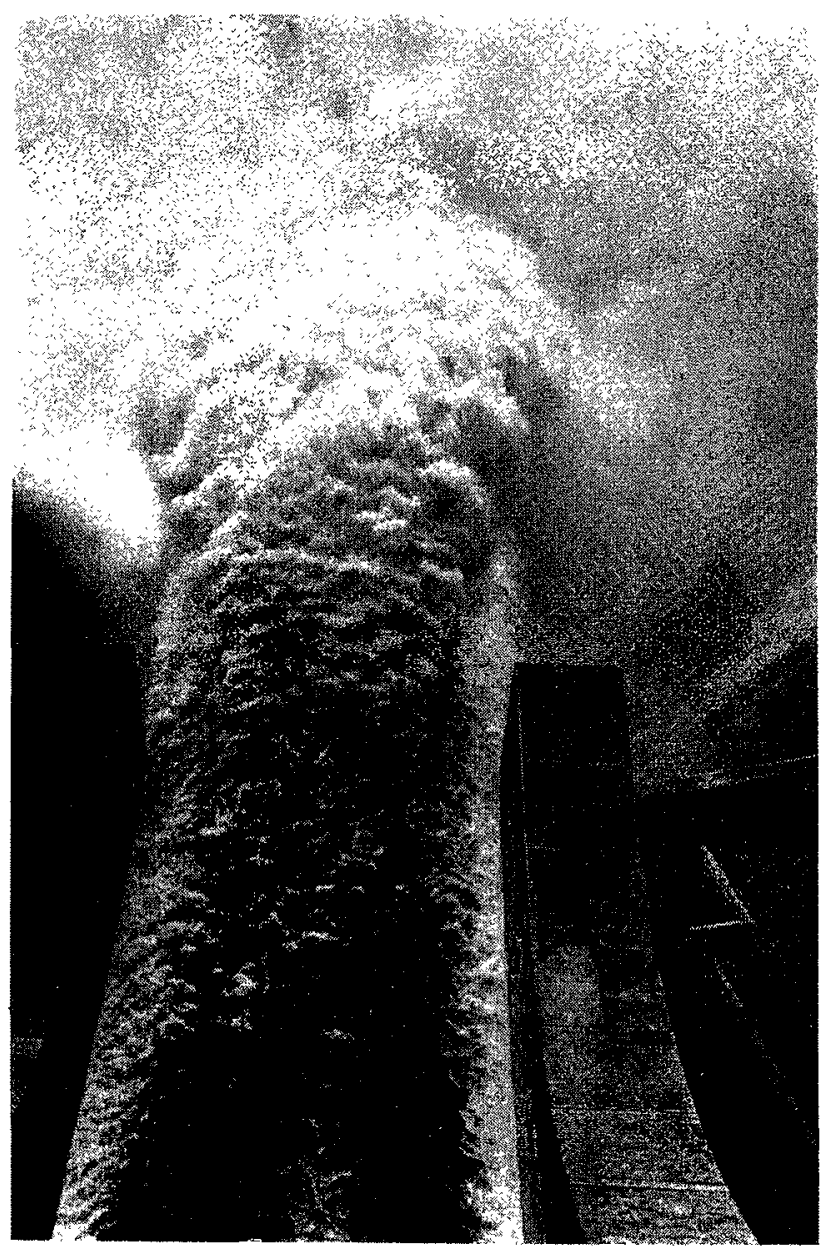

Fic. 13. $-\mathrm{Q}=230 \mathrm{~m}^{3} / \mathrm{s}$.

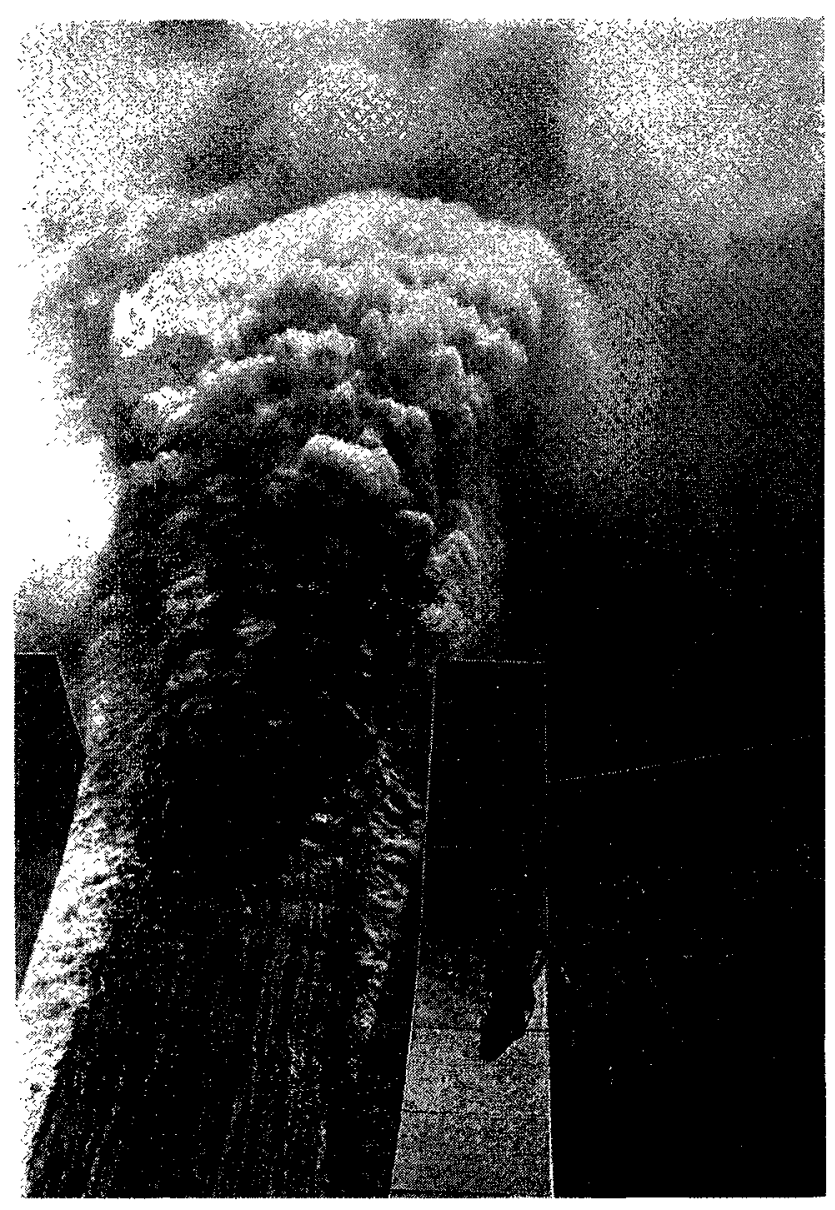

Fig. 14. -- $Q=350 \mathrm{~m}^{8} / \mathrm{s}$. 


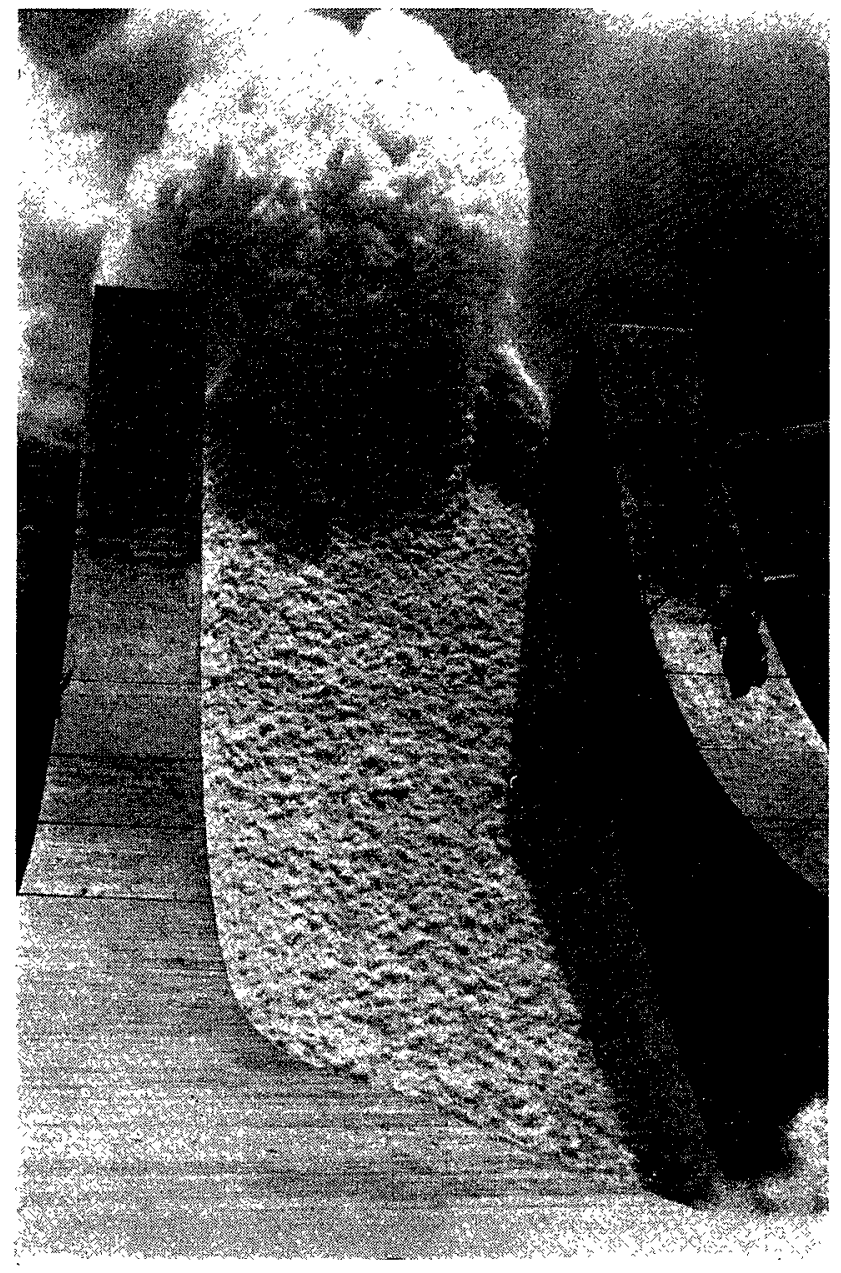

Fig. 11. $-Q=50 \mathrm{~m}^{3} / \mathrm{s}$.

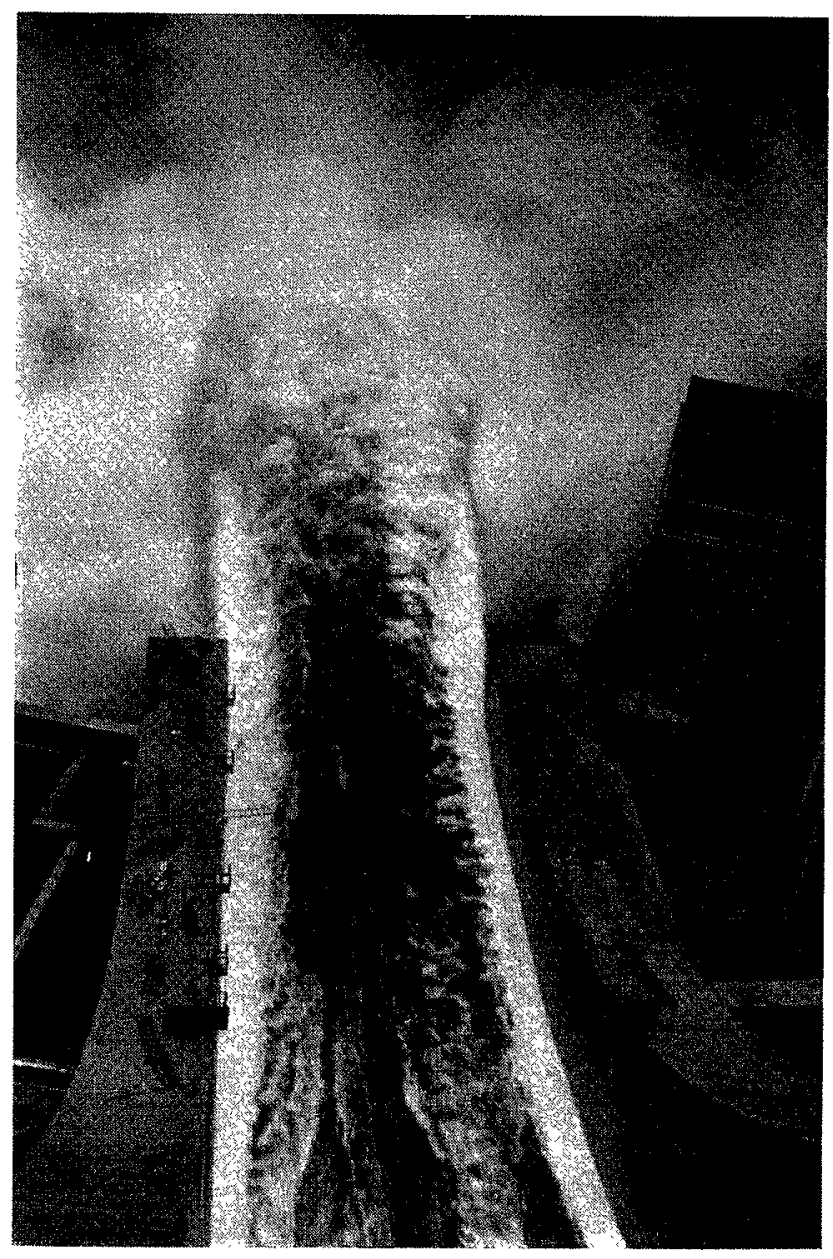

FlG. 15. $-Q=480 \mathrm{~m}^{3} / \mathrm{s}$.

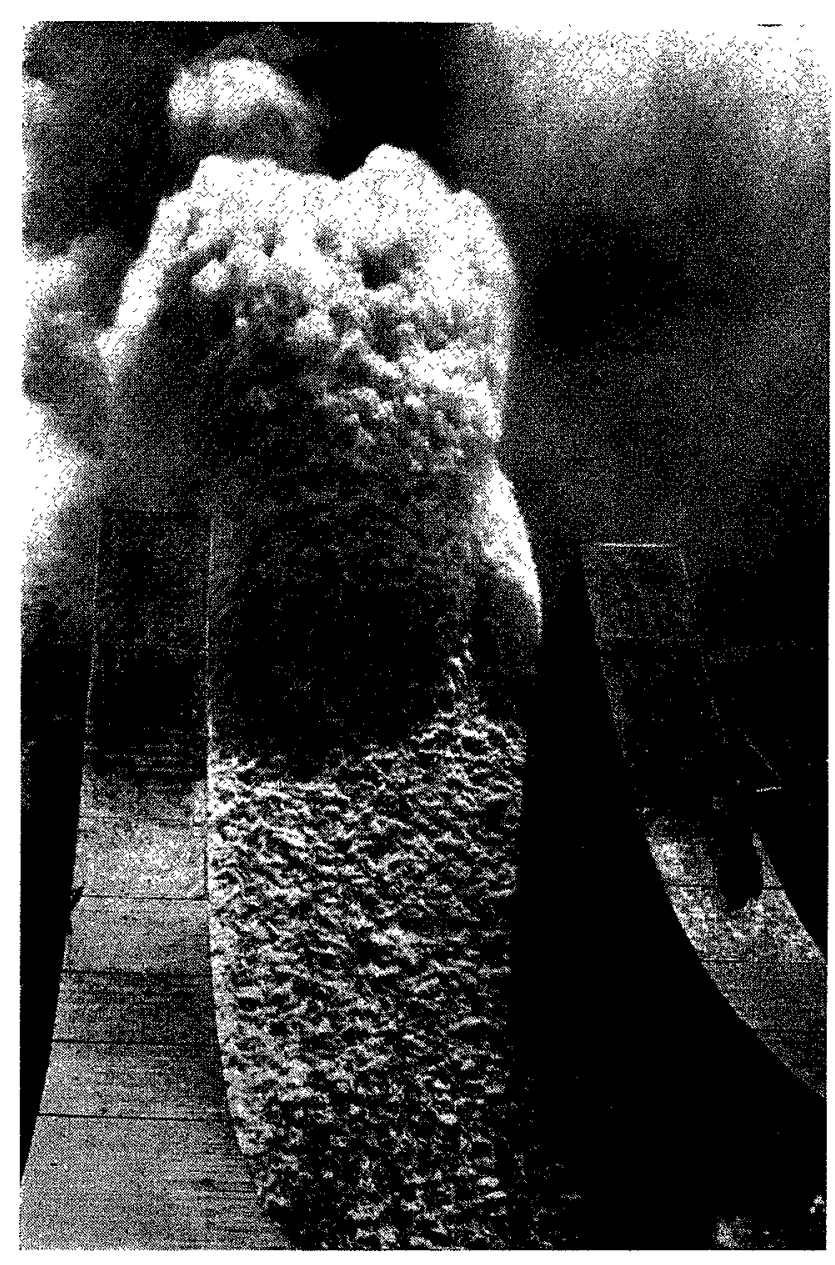

Fig. 12. - Débit non mesuré.

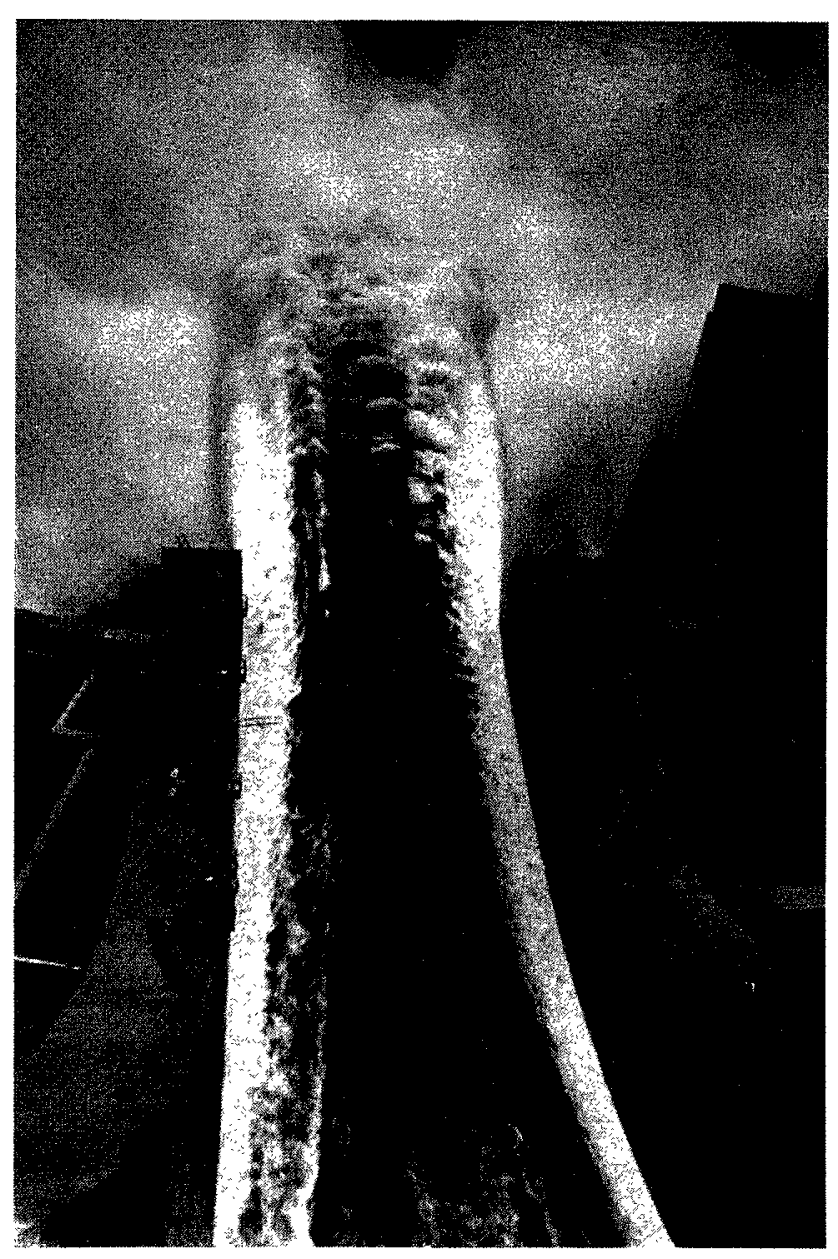

Fig, $16,-\mathrm{Q}=600 \mathrm{~m}^{3} / \mathrm{s}$. 


\section{PIANCHE IV. - FORMATION DE L'B́MULSION}

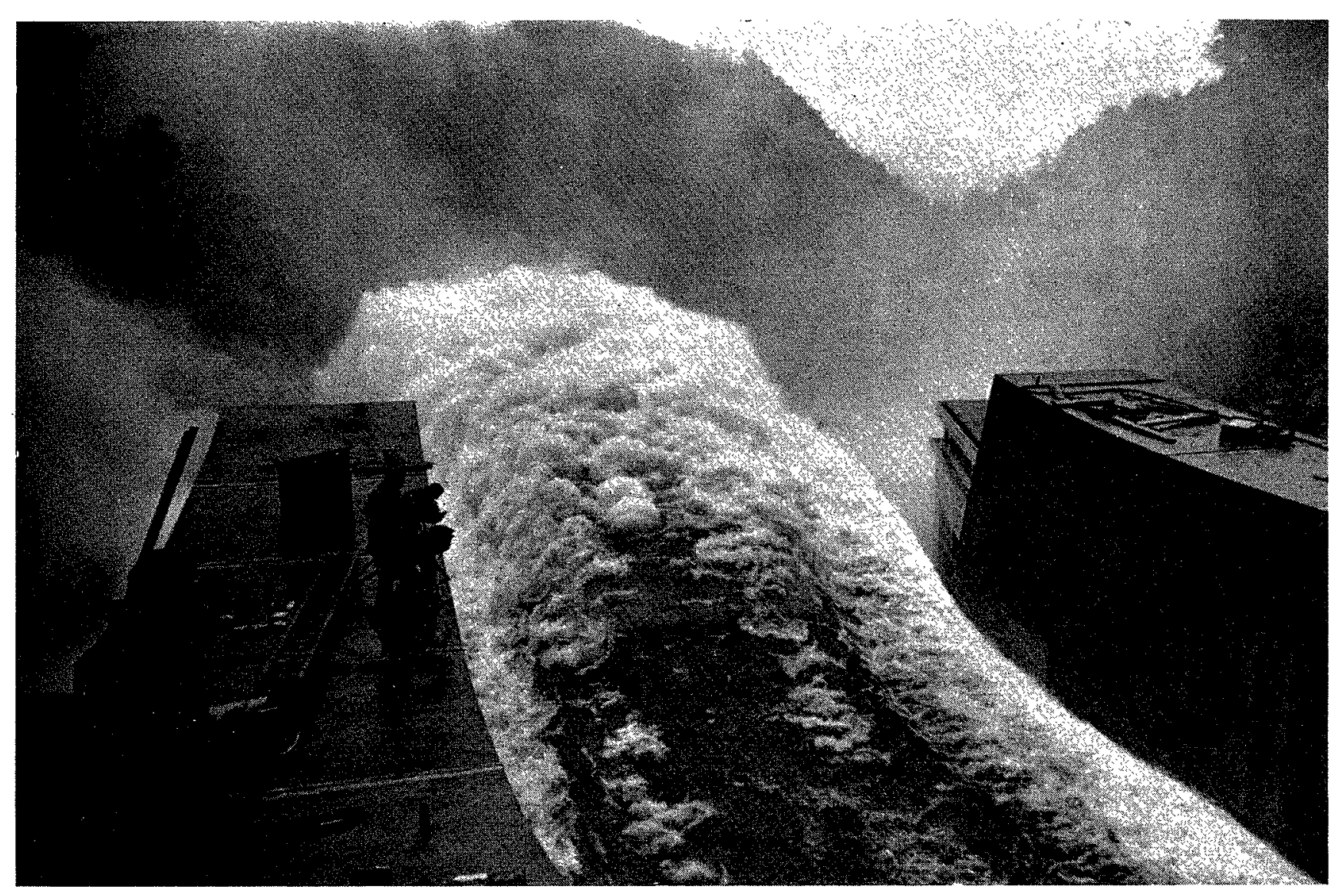

Fig. $17 .-$ Chastang $\left(270 \mathrm{~m}^{3} / \mathrm{s}\right)$.

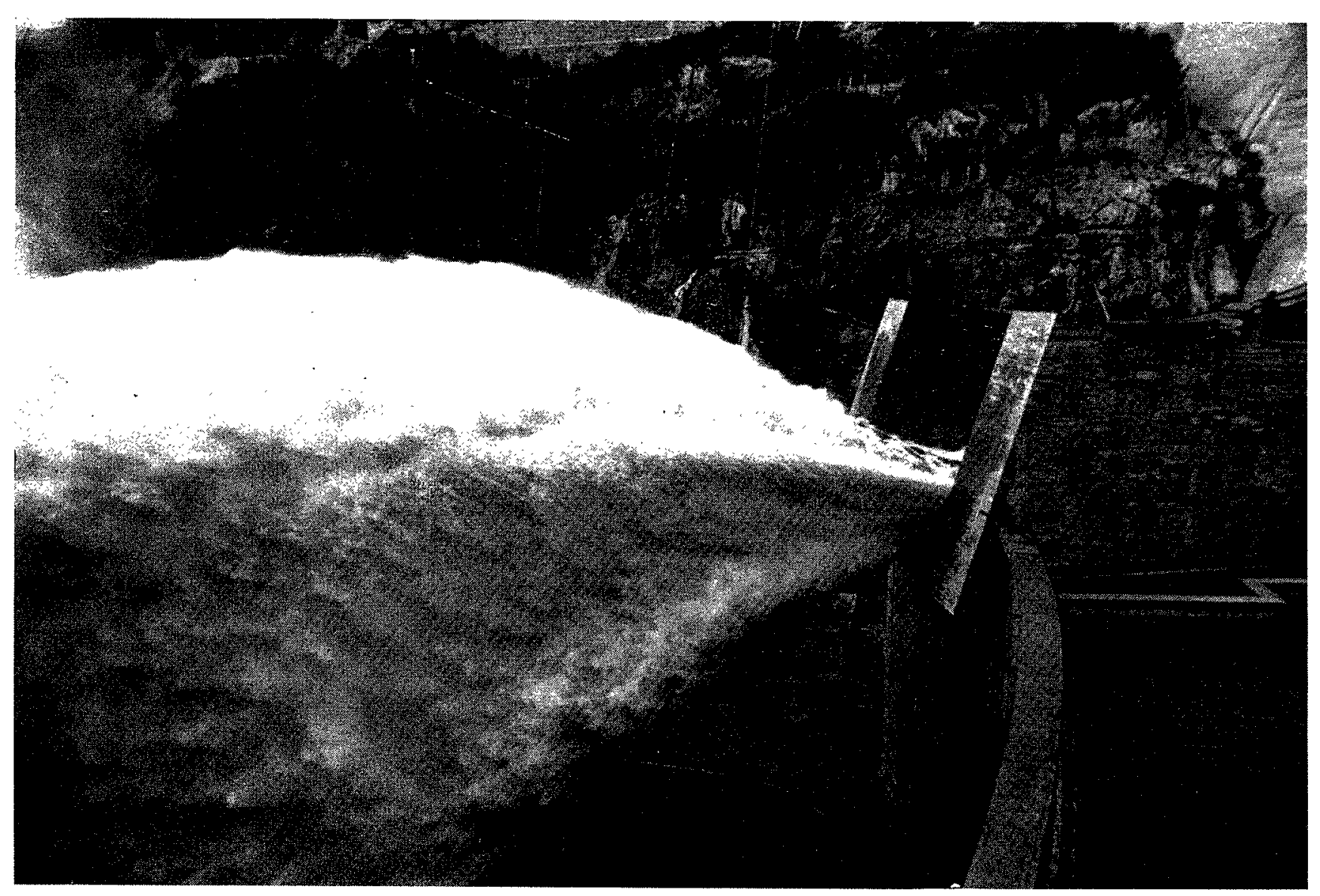

Fili. $18 .-$ Chastaxi $\left(270 \mathrm{~m}^{3} / \mathrm{s}\right)$. 


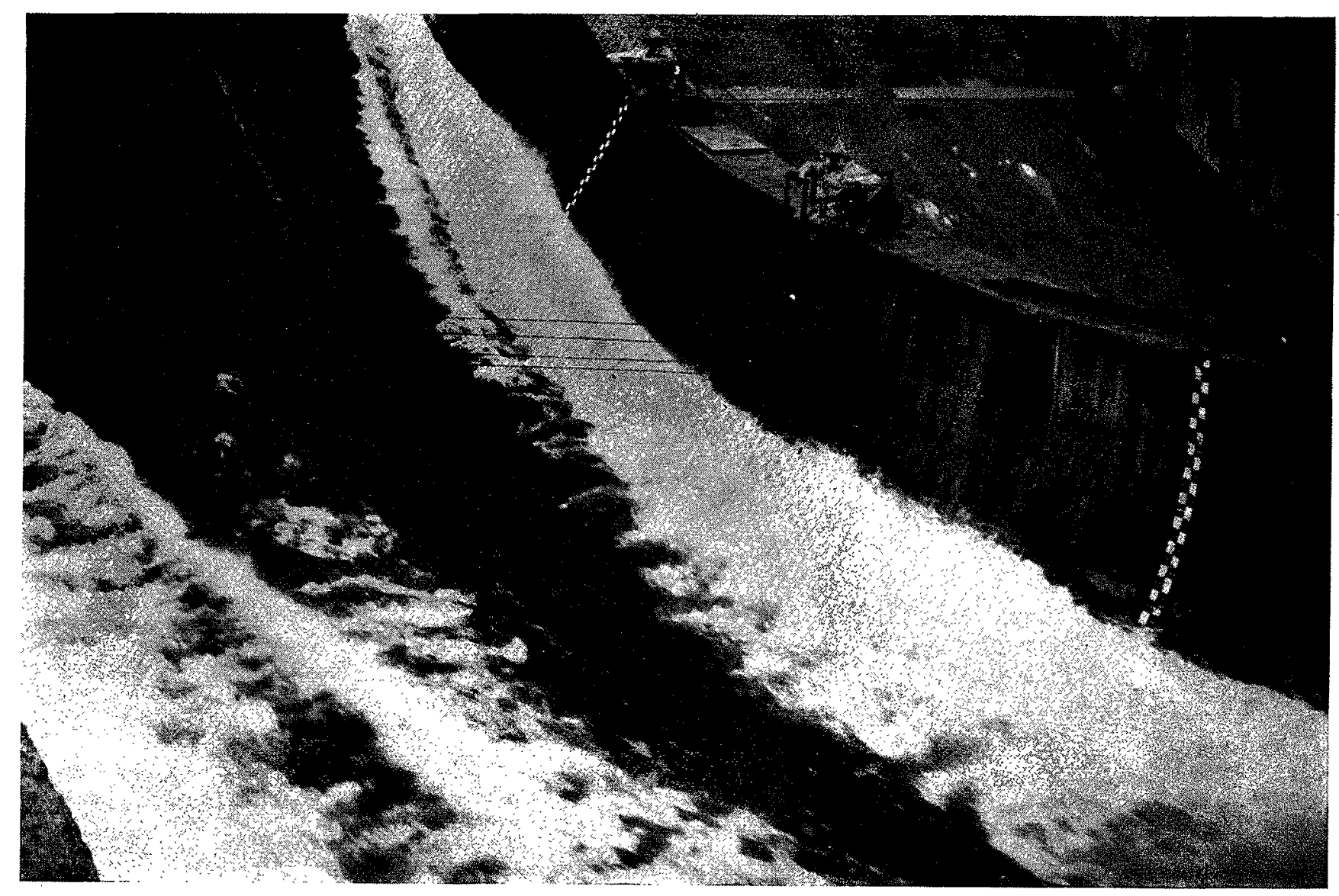

Fig. 19. - Saint-Etienne-Cantalès $\left(230 \mathrm{~m}^{\mathrm{s}} / \mathrm{s}\right)$.

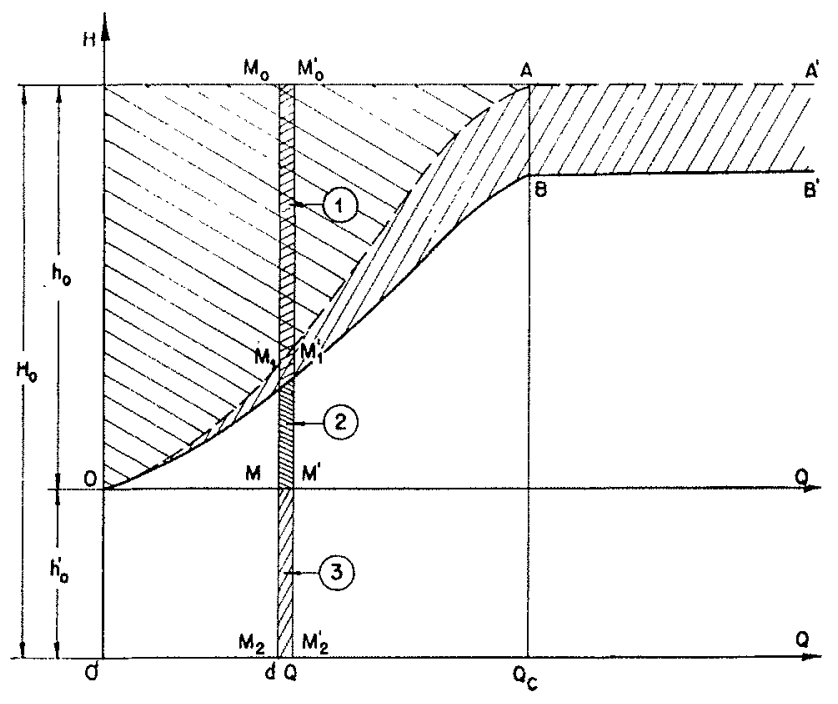

FIG. 20

$h_{0}=$ hauteur de chute sur l'ouvrage.

$h^{\prime}=$ hauteur de chute libre.

$1=$ énergie dissipée sur le coursier.

$2=$ énergie cinétique à la sortie de l'ouvrage.

$\mathbf{3}=$ énergie potentielle à la sortie de l'ouvrage.

$O A A^{\prime}=$ écoulement sur un coursier de largeur infinie. $\mathrm{OBB}^{\prime}=$ écoulement sur un ouvrage réel.

On a reporté sur le diagramme de la figure 20 l'évolution de la charge $H$ relative au filet liquide compris entre les lignes de courant $Q$ et $Q+d Q$ :
- Le segment $\mathrm{M} \mathrm{M}_{0}$ représente la charge disponible à l'amont rapportée à l'extrémité aval du coursier: cette charge est la même pour les différents filets liquides. Le lieu de $\mathrm{M}_{0}$ est done l'horizontale $\mathrm{H}=\mathrm{H}_{0}$;

- Le segment $M M_{1}$ représente la charge résiduelle à la sortie de l'ouvrage dans le cas de l'écoulement à deux dimensions : pour les lignes de courant relatives à un débit $\mathrm{Q}>\mathrm{Q}_{c}$, le filet liquide traverse l'ouvrage sans rencontrer la couche limite et la perte de charge $\Delta \mathrm{H}=\mathrm{M}_{1} \mathrm{M}_{0}$ s'annule. Le lieu de $M_{1}$ est donc confondu avec celui de $M_{0}$. Pour les lignes de courant relatives à $\mathrm{Q}<\mathrm{Q}$, la charge résiduelle croît avec $\mathrm{Q}$ par suite du déplacement du point $c$ vers l'aval. Cette charge résiduelle étant pratiquement nulle aux très petits débits, le lieu de $M_{1}$ est une certaine courbe $\mathrm{O} A$.

Dans le cas d'un ouvrage réel, de largeur finie, il faut tenir compte de l'influence des parois latérales qui se fait sentir de manière identique pour tout filet liquide $d Q$ situé au-dessus de la ligne de courant $\mathrm{Q}=\mathrm{Q}_{c}$. L'énergie résiduelle est donc constante mais inférieure à $h_{0}$. La courbe $\mathrm{O} \mathrm{A} \mathrm{A}^{\prime}$ devient $O \mathrm{~B} \mathrm{~B}^{\prime}$, le décalage entre les deux courbes étant fonction de la forme de la section transversale de l'ouvrage.

A partir de ce diagramme de l'évolution de la charge des différents filets liquides constituant 
l'écoulement, on obtient par intégration le diagramme du bilan d'énergie pour un débit évacué $Q$.

En effet, l'énergie contenue dans le filet $d Q$ possédant la charge $H$ est, par définition, $d \mathrm{~W}^{r}=\pi \mathrm{H} d \mathrm{Q}$. D'autre part, l'élément d'aire $M M^{\prime} M_{1} \mathrm{~N}_{1}^{\prime}$ (fig. 20) a pour surface $\mathrm{H} . d Q$.

On a done :

$$
d \mathrm{~W}=\omega \text { aire } \overline{\mathrm{M} \mathrm{M}^{\prime}} \overline{\mathrm{M}_{1}} \overline{\mathrm{M}_{1}^{\prime}}
$$

Par suite, l'énergie contenue dans le débit Q a la sortie de l'ouvrage :

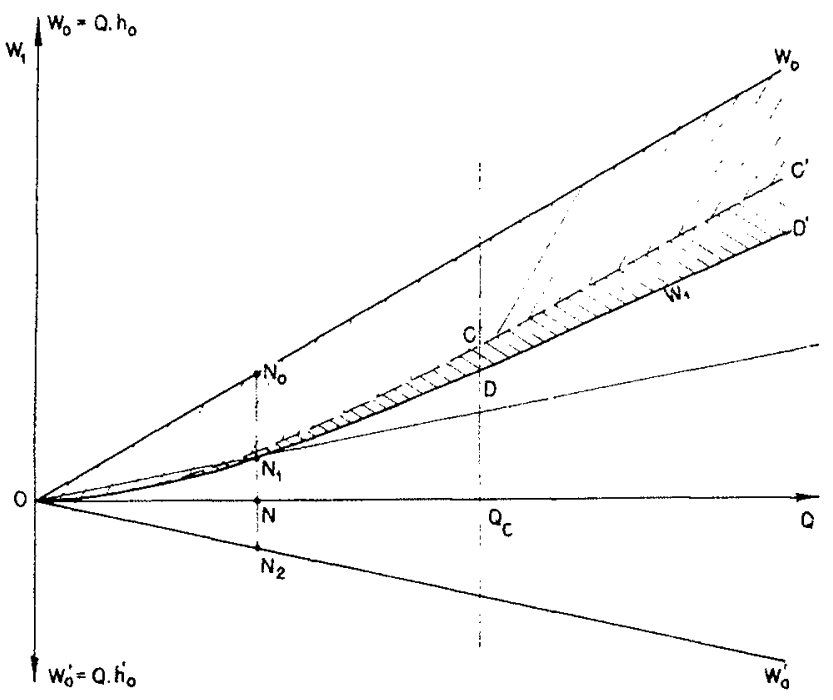

FiG. 21

$h_{0}=$ hauteur de chule sur l'ouvage.

$h_{0}^{\prime}=$ hauteur de chute libre.

$\mathrm{N}_{\mathrm{i}} \mathrm{N}_{0}=$ énergie dissipée sur le coursier.

$\bar{N} \overline{N_{1}}=$ énergie cinctique à la sortie de l'ouvrage.

$\mathbf{N} \mathrm{N}^{\prime}=$ énergie potentielle a la sortie de l'ouvrage.

(O) $D^{\circ}=$ écoulement sur un coursier de largenr infinie.

o $C^{\prime} C^{\prime}=$ écoulement sur un ouvrage réel.

$$
\mathrm{W}_{1}=\int_{0}^{\mathrm{Q}} \pi \mathrm{H} d \mathrm{Q}=\text { w.aire } \overline{\overline{\mathrm{OMM}}}
$$

D'autre part, l'énergie disponible dans le débit $Q$ à l'entrée de l'ouvrage est :

$$
\mathrm{w}_{0}=\int_{0}^{\mathrm{Q}} \omega h_{0} d \mathrm{Q}=\pi h_{0} \mathrm{Q}
$$

On a do même l'énergie disponible dans le jet:

$$
W^{\prime}{ }_{0}=\bar{\omega} h_{0}^{\prime} \cdot Q
$$

Sur le diagramme de la figure 21 , on a reportí ces divers termes :

$$
\begin{aligned}
& \overrightarrow{\mathrm{NN}_{0}}=\mathrm{W}_{0} \\
& \overline{\mathrm{N} \mathrm{N}_{1}}=\mathrm{W}_{1} \\
& \overline{\mathrm{N}_{2} \mathrm{~N}}=\mathrm{W}_{0}^{\prime}
\end{aligned}
$$

Le lieu de $\mathrm{N}_{1}$ est la courbe $\mathrm{O} \mathrm{C}_{\mathrm{C}} \mathrm{C}^{\prime}$ pour un écoulement à deux dimensions, la courbe O D D' dans le cas d'un ouvrage de largeur finie. L'énergie dissipée sur l'ourrage, représentée par le segment $\mathrm{N}_{1} \mathrm{~N}_{0}$, ne croît que tries faiblement au-delà du

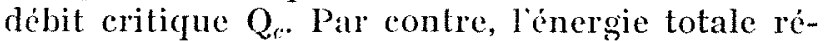
siduelle à la sortie du coursier rapportée au niveau aval est représentée par le segment $N_{2} N_{1}$.

L'allure de la courbe O D D' montre que cette énergie croît comme une puissance du débit supérieure à l'unité. Si l'on compare cette courbe á la variation linéaire représentée par la droite $\mathrm{ON}$, on voit qu'il ne saurait être question d'extrapoler aux forts débits un pourcentage de pertes de charge relevé pour un débit faible ou moyen, la proportion d'énergie dissipéc sur le coursier diminuant lorsque le débit crô̂t.

\section{DEUXIÈ M E PART I E}

\section{LES MESURES REALISEES SUR LES EVACUATEURS DE CRUE DE SAINT-ETIENNE-CANTALĖS ET DE CHASTANG}

Ces essais sur des ouvrages réels avaient pour but de permettre une evaluation expérimentale sérieuse de la valeur de la perte de charge sur les évacuateurs de surface et, d'une manière plus générale, d'aboutir à une meilleure connaissance des lois de l'écoulement sur de tels ouvrages.

L'évaluation des pertes de charge nécessitait la détermination de la vitesse moyenne dans plusieurs sections de l'écoulement au moyen de la mesure de la section mouillée et du débit. En outre, on a cherché à préciser la distribution des vitesses dans une section de l'écoulement par des mesures direcles au voisinage de la surface et $d u$ fond; on a mesuré enfin la pression en certains points du radier.

Ces mesures ont été réalisées par la Division des Essais Extérieurs du Service des Etudes et Recherches Hydrauliques Iors de déversements provoqués sur les barrages de SaintEtienne-Cantalès et de Chastang. 
Le premier essai fut efrectuć à lusine de SaintEtienne-Cantales en décembre 1950 . Il n'y s’agissait à vrai dire que d'une mise au point du matériel étudić pour déterminer les profils en travers de la nappe diversante. Un essai plus complet fut effectué en février $1952 \mathrm{sur}$ le déversoir de l'usine de Chaslang pour lequel un modile reduit avit été étudié au Laboratoire National d'Hydraulique et dont le coursier avait pu ètre muni dorifices communiquant avec l'exlérieur et destinés a la mesure des pressions el des ritesses.

Ce sont les résultats obtenus au cours de ces deux essais que nous présentons ci-apris.

\section{A) Méthodes de mesure employées.}

\section{$1^{\circ}$ Mestre DES VITESSES MOYENNES} DANS LASS DHFERENTES SECTIONS
a) Détermination du profil en travers ar la nappe dénersante:

Jusqu’à présent, les seuls relevés concernant la configuration de l'ecoulement dans les nap- pes diversantes d'evacuatcurs de crues avaient che obtenus au moyen d'ichelles limmimétriques installees sur les bajoyers du deversoir. Celte méthode ne permeltail pas de délerminer le profil transwersal de la nappe et la precision des releves aux échelles s'avérait on outre relativement lablle. ea sgard aux flucluations du plan d'eau an voisinage des parois. Le premier objectil de la bivision des Essais Extéleurs a ble de mettre at point un dispositif permetlant commodément de determiner avere une bonne precision $(-1$ is 2 am pres) le profil en travers de la nappe suivant plusicurs seclions transversales.

Nous avons envisatic de realiser dabord uno poutre amovible cquipe do plusieurs postes de mesure, el susceptible d'ètre déplacée le long du coursier. Ce procédé présentait de sérieuses difficultés en raison des grandes vitesses de lair motrane a la surface de la nappe. Apres avoir 'nvisage l'utilisation de la photographie slirioscopique, nous nous sommes orientés vers un releve topographique par triangulation.

En chaque section transversale de mesure sont installes deux thiodolites fixis au-dessus des deux bajoyers du coursier (lig. 22). Les cereles verticaux de ces theodolites btant amenes dams un meme plan of la distance entre leurs axes ho-

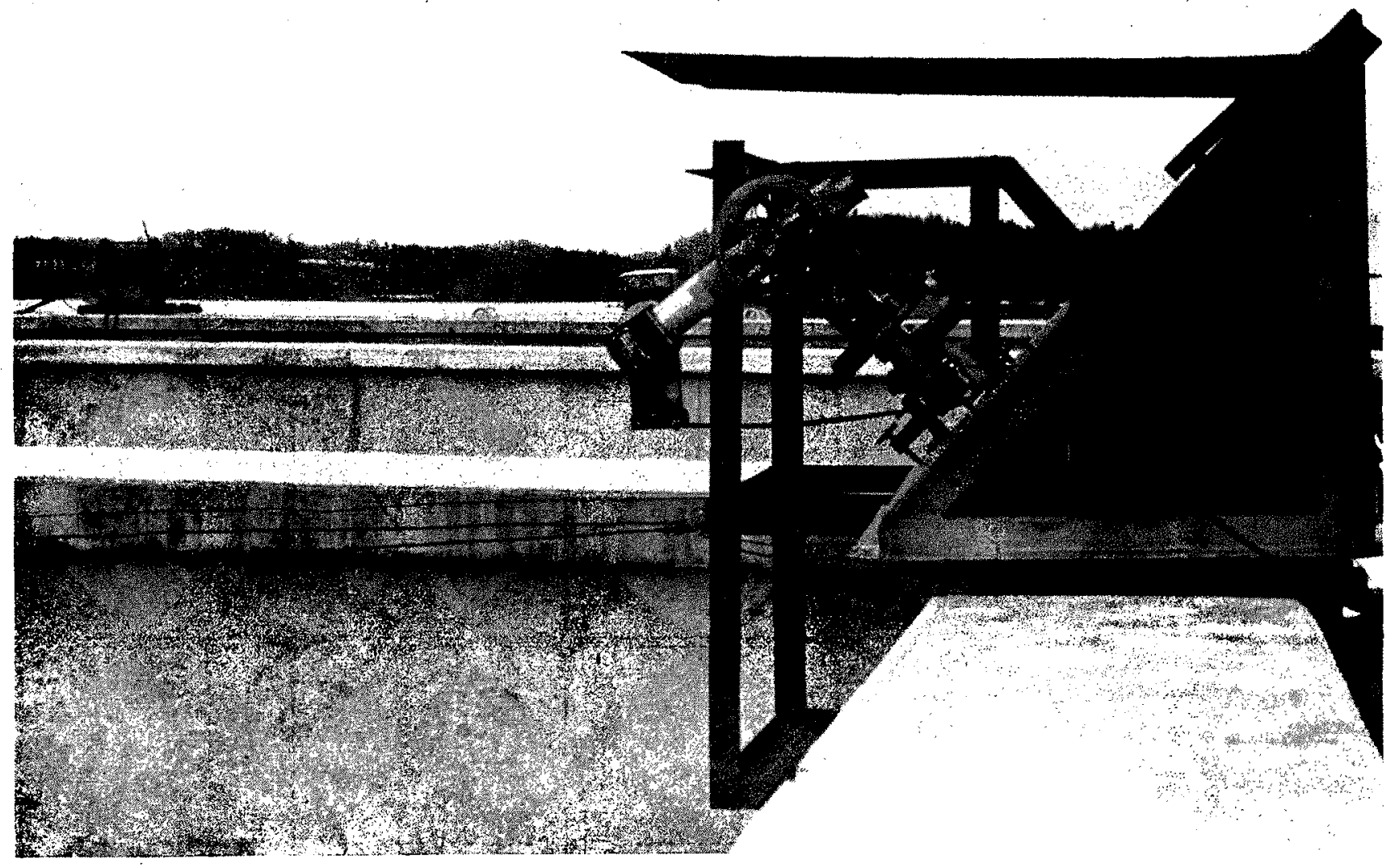

Fig. 22. - In des theobolites de mesure. 
rizontaux étant mesurée, la position d'un point quelconque du plan précité est déterminée par simple lecture d'un angle sur les deux appareils. La seule difficulté d'utilisation de cette méthode est due à l'impossibilité de matérialiser le point sur lequel les deux appareils doivent effectuer la visée. C'est pourquoi nous avons remplacé les lunettes optiques des deux théodolites par des projecteurs axiaux munis d'une croix particulièrement fine susceptible d'ètre projetée sur la surface du liquide. II suffit alors de réaliser la coïncidence des croix pour obtenir les coordonnées de l'un des points cherchés. Ces visées peuvent être effectuées successivement sur le radier du coursier sans que le déversoir ne fonctionne, puis pour un débit donné, ce qui permet de déterminer en chaque point de la section la valeur du tirant d'eau.

Cette méthode simple, dont le seul inconvénient est de nécessiter des essais de nuit, a exigé de nombreuses mises au point notamment pour obtenir la projection d'une croix suffisamment fine et nette sur un écran fluide recevant des rayons lumineux dobliquité variable à des distances de la lunette de projection elles aussi variables. (La mise au point de limage en fonction de la distance aurait en effet nécessité des dispositions très coûteuses en raison de la difficulté de réaliser ce réglage sans modifier laxe optique du projecteur.)

Ainsi que nous l'avions admis, à priori, la netteté de l'image peut ìtre parfaitement réalisce dans les écoulements d'eaux fortement émulsionnées, offrant de ce fail une surface analogue à celle d'un écran de projection. Mais nous avons constaté en outre que dans les écoulements d'eaux claires présentant des stries fines telles que l'on en rencontre dans les parties supérieures des évacuateurs de crues, l'image à la surface est encore très nette bien que pour les faibles tirants d'eau l'on aperẹoive souvent simultanément les deux images projetées, l'une sur la surface du plan d'eau et l'autre sur le radier. (Dans ce cas, nous avons même tenté d'utiliser cette double image pour déterminer directement le tirant d'eau, mais la précision de cette mesure est très modeste.)

Les plans de visée optique coïncidaient avec le plan transversal perpendiculaire au radier du coursier. Lorsque cela n'était pas possible, le plan de visée était le plan vertical.

Pour chaque section de mesure, il a été procédé à un double balayage du profil. Au cours du premier balayage, l'un des postes réglait successivement son théodolite à des angles fixés à l'avance, compte tenu du tirant d'eau prévu; l'observateur du second poste assurait la coïncidence de sa croix avee celle du poste opposé. Au cours du second halayage, les fonctions des deux postes étaient inversées. Deux séries de

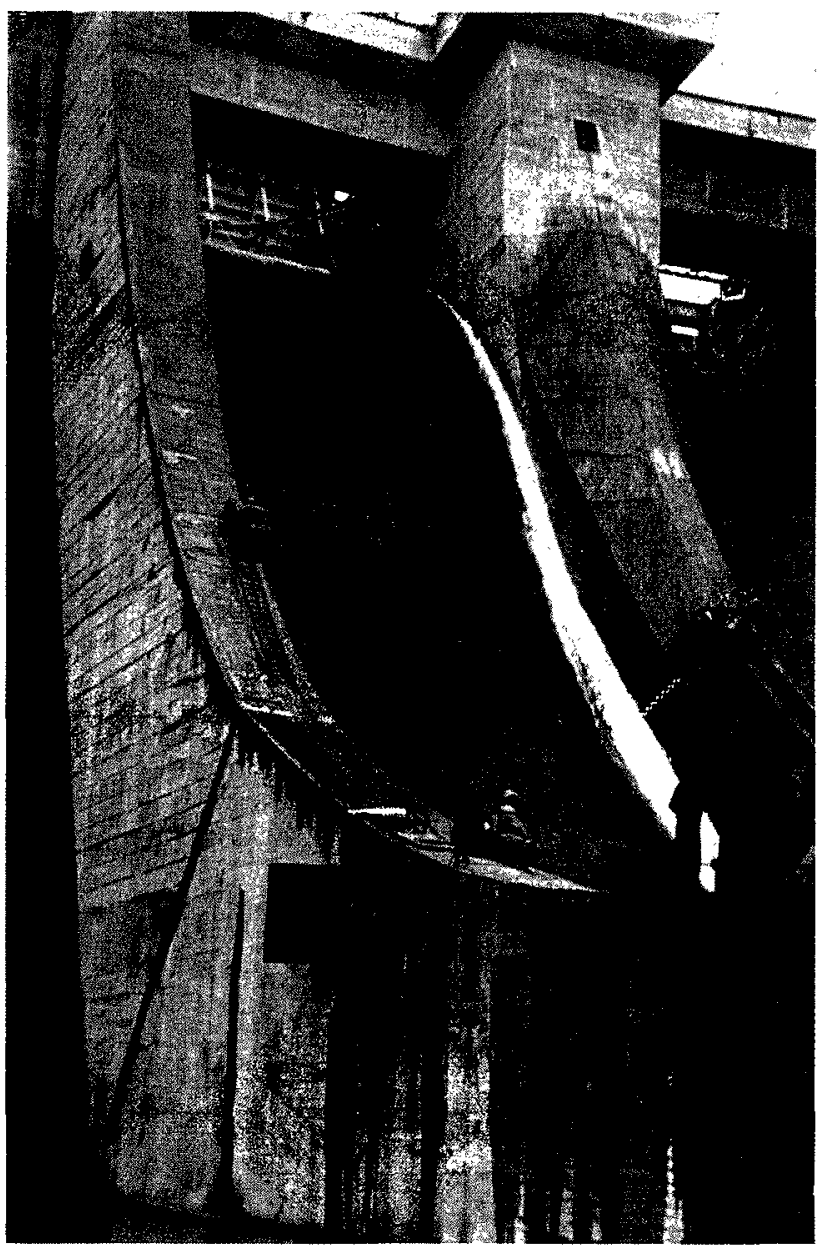

Fri. 23. - Vue de quelques postes de mesure durant un deversement.

6 à 7 points de mesure étaient ainsi effectuces (fig. 23).

Les relevés effectués sur le radier ont permis de constater que les erreurs sur les altitudes mesurées étaient de l'ordre de $\pm 1 \mathrm{~cm}$, ce qui correspondait à une indétermination de lecture des appareils de l'ordre de $\pm 2 / 100$ grade. Les visées sur la nappe liquide étaient légèrement moins précises en raison surtout des fluctuations relativement importantes du plan d'eau. Toutefois, l'examen des différents points obtenus successivement sur un même profil dans des sections où plusieurs doubles balayages ont été effectués ont permis de constater que l'erreur accidentelle ne dépassait jamais $\pm 5 \mathrm{~cm}$ et qu'elle était en moyenne de l'ordre de $2 \mathrm{~cm}$.

\section{b) Mesure des débits :}

Pour l'usine de Chastang, le débit déversé était déterminé à partir d'essais sur modèle réduit en fonction de la hauteur de levée de la ranne secteur et de l'altitude du plan d'eau de la retenue.

Ce débit a pu être contrôlé par la station 
d'Argentat située sur la Dordogne à une dizaine de kilometres à l'aval de l'usine de Chastang.

En cetle station, en effet, la variation de débit accusait un palier d'une durce d'une heure environ, pendant lequel le Service des Forees $\mathrm{Hy}^{-}$ drauliques de la $4^{\mathrm{c}}$ Circonseriplion Electrique put effectuer un jaugeage au moyen d'un moulinet suspendu. Le débit mesuré au cours de ce jaugeage était égal, à moins de $4 \%$ près, à celui déduit de la lecture à l'échelle d'Argentat et à celui déduit de la Ievée de vanne de l'usine de Chastang.

Le déversoir de l'usine de Saint-Etienne-Canlales ne possédant pas de tarage de la courbe de débit en fonction de la levée de vanne, il fut procédé à une détermination du débit par méthode volumétrique grâce à la capacité constituée par le bassin de compensation de Nêpes. Le niveau du plan d'eau dans ce bassin clait mesuré au moyen de trois limnigraphes et de cing échelles limnimétriques. Ia montée du plan d'eau correspondant au passage du plein débit de l'ćvacuateur pendant une durée de 30 minutes était de 2 metres environ; compte tenu de l'approximation sur le levé topographique de la relenue, l'erreur sur le débit était inférieure à \pm 2 à $3 \%$.

\section{$2^{\circ}$ RÉPARTition DES Vitesses}

\section{(t) Vitesses à la surface de la nappe:}

Les vitesses en surface ont été relevées à lusine de Chastang au moyen de flotteurs de surface constitués par des réservoirs vides de diverses formes et d'une capacite de deux litres environ. Ces réservoirs étaient lancés à partir des guideaux ou du couronnement du barrage. Leur position instantanée était repérée au moyen d'appareils cinématographiques dont la vitesse de déroulement de film élait soigneusement contrôlée. Chaque flotteur apparaissait successivement dans le champ de l'appareil sur 4 à 5 images successives et les courbes des distances parcourues en fonction du temps étaient très sensiblement linéaires.

L'étalonnage des distances parcourues par les llotteurs, en fonction des déplacements sur les images photographiques, était obtenu au moyen d'une grille d'ćtalonnage, constituée par un quadrillage rigide placé sur le coursier et photographić avant déversement.

Trois appareils mesuraient successivement les vilesses des flotteurs en trois sections coïncidant sensiblement avec trois sections de délermination du profil de la nappe déversante.

\section{b) Vitesses an noisinage du conrsier:}

Les vitesses au voisinage du coursier ont été relevées au moyen de tubes de Pitor. Compte tenu des eflorts importants susceptibles de s'exercer sur ces appareils, pour des vilesses de l'ordre de $20 \mathrm{~m} / \mathrm{s}$, ceux-ci étaient constilues par un support cylindrique massif de section pisciforme, scellé dans le radier el muni de deux couples de prises dynamiques el staliques prévues respectivement $\dot{a} 5 \mathrm{~cm}$ et a $15 \mathrm{~cm}$ au-dessus du coursier (fig. 24).

Cette disposition devait permettre de diterminer le gradient de vilesse au voisinage du coursier et d'évaluer par suile un ordre de grandeur de la rugosité relative des parois du deversoir.

Les prises de pression ilaient réunies, au moyen de canalisations en canoulchouc, à des manometres différentiels à colonnes de mereure installés dans une salle de mesure à l'intéricur. de l'usine.

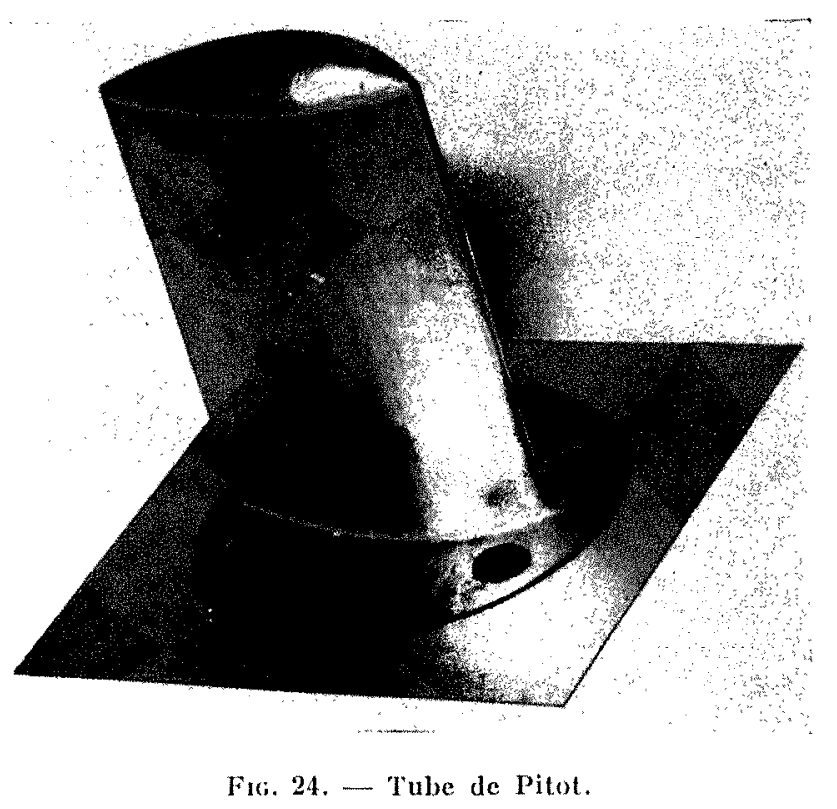

Ces manomères comportaient deux tubes de verre raceordés par un U en caoutchoue. Ies plaques-supports des tubes pouvaient elles-memes coulisser sur des tubes d'échafaudage, ce qui permettait de mesturer toute dénivellation comprise entre $1 \mathrm{~cm}$ et $5 \mathrm{~m}$ de mercure.

Les pressions étaient très fluctuantes pour les faibles valeurs du débit déversć, mais restaient pratiquement constantes $(\dot{a} \pm 1 \mathrm{~cm} / \mathrm{Hg}$ près $)$ pendant toute la durée de l'écoulement à débit constant.

\section{$3^{\circ}$ MESURE DES PRESSIONS SUR LE RADIEI}

Les pressions s'exerçant sur le radier ont été relevées en cing points répartis sur le coursier, au voisinage des emplacements de mesure des vitesses. 
Chaque prise de pression était constituce par une plaque circulaire, situce dans le plan du coursier, percée de trous de $2 \mathrm{~mm}$ de diametre et reliće à un tube piézométrique à mercure identique aux précédents.

A titre d'essai, nous arous hranche sur l'une des prises un manomètre à membrane utilisant une transmission électrique, dont l'itude était en cours. Cet appareil comportait une membrane circulaire tenduc dont la deformation ilait enregistrée par un potentiomotre d'une course tolale de $0,5 \mathrm{~mm}$ pouvanl être mesurée à $\pm 0, \overline{5} .10^{-2} \mathrm{~mm}$ pres. Dans la gamme d'utilisation de l'appareil expérimental, pour les lables valeurs du débit. ce manomètre s'est révéle pratique el précis. Ses indications coüncidaient tris sensiblement avec celles du piézomèlre à colonnes de mercure.

\section{$4^{\circ}$ OngaNisatiox DES FSSAIS}

Ces essais mettaient en ourre un matériel relativement important el necessitaient un effoclif en personnel dépassanl largement les possibilites de la Division des Essais Exterieurs a l'usine de Chastang par exemple, la nappe déversante était déterminée suivant cinq sections transversales, tandis que les pressions el les vilesses étaient mesurées en ciny points. Compte tenu de la nécessité de relayer le personnel d'observation du plan d'eau en raison de la rigueur du temps à l'époque des essais, il fallait disposer à celte usine de 25 observateurs. Si l'on ajoute à cela la nécessité de réduire au maximum la duréc du deversement pour limiter autant que possible les pertes d'eau ct l'intérêt d'une surveillance permanente des relevés obtenus en cours d'essai par un calcul rapide des resultals obtenus, on peut comprendre la minulic avec laquelle ont dù être conduits les travaux de preparation de ces essais (entrainement du personnel d'observalion par des relevés nocturnes la veille de l'essai, synchronisation des relevés aux différents postes de mesure, réalisation d'un système de calcul sommaire très rapide des lirants d'eau, etc.). En fait, grâce au personnel et aux moyens mis à notre disposition par le Groupe de Production Hydraulique «Dordogne» ct la Région d'Equipement Hydrauliọue « Massif Central II », le régime stable de diversement n'a été maintenu que pendant trente minules

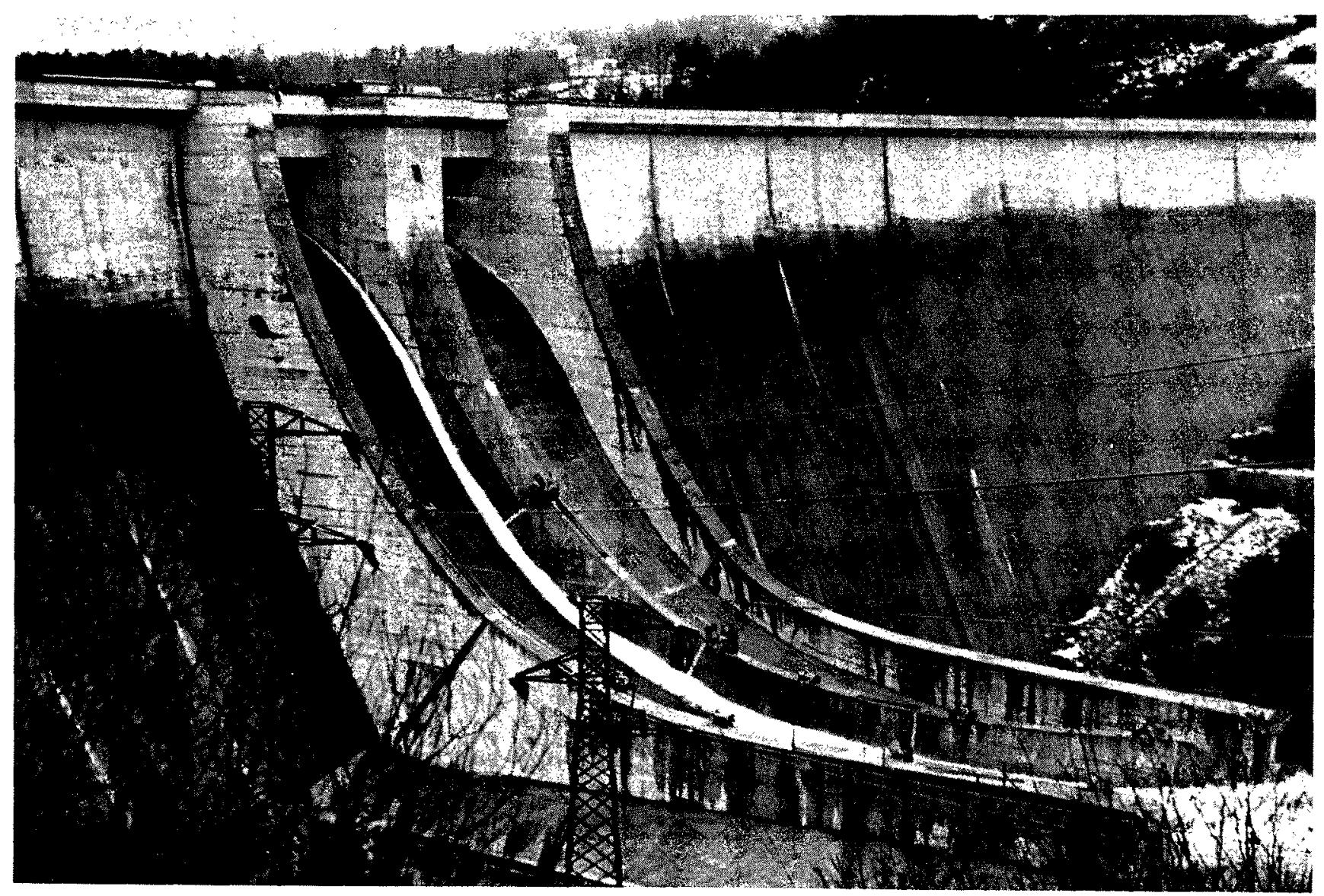

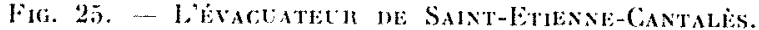


environ à l'usine de Saint-Etienne-Cantales, et ane heure à liusine de Chastang.

Ainsi que cela a été exposé précédemment, deu: essais ont été eflectués : le premier à l'usine do Saint-Etienne-Cantalès en décembre 1950 et le second à l'usine de Chastang en février 1952.

\section{B) Résultats de mesure.}

\section{1* Usine de Sant-ETifine-Cantalis}

L'évacuateur de crues de l'usine-barrage de Saint-Etienne-Cantales sur la Cìre, prévue pour un débit de $1.000 \mathrm{~m} / \mathrm{s}$, est assurée par la levéc de deux vannes-secteurs, obturant deux pertuis de $11,7 \mathrm{~m}$ de largeur et de $7,70 \mathrm{~m}$ de hauteur, alimentant un déversoir du lype saut de ski, installé sur le toit de l'usine (fig. 25).

On s'est borné dans cet essai à mesurer les vitesses moyennes de l'écoulement.

Les relevés de la hauteur de lame ont été effectués pour un débit de $230 \mathrm{~m}^{3} / \mathrm{s}$, concentré dans un seul pertuis, en six profils uniformément répartis sur le coursier à un intervalle moyen de 15 mètres.

Les résultats des caleuls des hauteurs moyennes et des surfaces mouillces sont rassembles dans le tableau ci-dessous.

VITESSES MOXENNES.

\begin{tabular}{|c|c|c|c|}
\hline $\begin{array}{l}\text { Numero } \\
\text { du profil }\end{array}$ & $\begin{array}{l}\text { Surface } \\
\text { mouillée } \\
\text { cu n- }\end{array}$ & $\begin{array}{l}\text { Hauteur } \\
\text { moyenne } \\
\text { cil m }\end{array}$ & $\begin{array}{l}\text { Vitesse } \\
\text { moyenne } \\
\text { en } 11 / \mathrm{s}\end{array}$ \\
\hline$A \ldots \ldots$ & 11,90 & 1,62 & 19,5 \\
\hline I3.... & 11,35 & 1,32 & 20,4 \\
\hline$(\ldots \ldots \ldots$ & 10.95 & 1,17 & 21,2 \\
\hline D).... & 10,95 & 1,08 & 21,2 \\
\hline $\mathrm{E} \ldots$ & 12,25 & 1,10 & 18,95 \\
\hline I $\ldots$. . & 18.95 & 1.65 & 12,25 \\
\hline
\end{tabular}

On peut eslimer que l'erreur sur la mesure des hauteurs est de l'ordre de $\pm 2 \%$. Le coursier n'etant pas de section constante, l'erreur moyenne sur la surface mouillée doit être de lordre de $\pm 2,5 \%$. Les vitesses moyennes sont donc connues à $\pm 2,5 \%$ près si lon suppose le

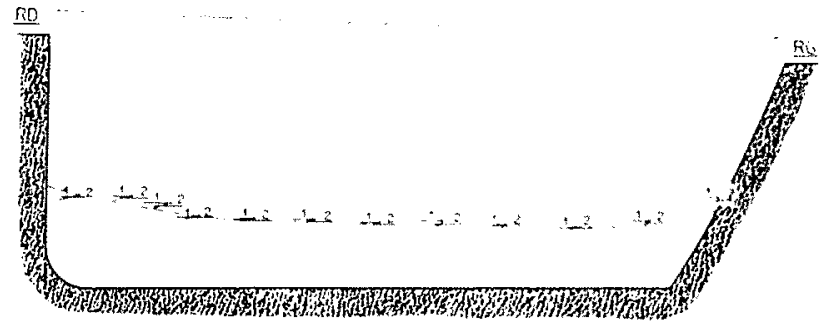

Fic. 26. - Saint-Etienne-Cantales.

Relevé transversal de la lame dans la section.

débit connu exactement. En fait, l'erreur sur le débit entraine une erreur systematique sur les vilesses morennes de loutes les sections dont il faudra tenir comple. Nous avons reproduit sur la figure 26 un relevé de profil: on peul y observer la faible dispersion des points expérimentaux qui correspondent it deux relerés successifs.

A partir de ces résullats, il est possible, comme on le verma plus loin, d'ivaluer les pertes de charge et de tracer la ligne de charge moyennant certaines corrections.

Les essais préliminaires nous ont done permis de nous assurer de la validite de la methode de mesure proposie et par suite de lancer l'essai prévu initialement à l'usine de Bort-les-Orgues et réalisé en fait à l'usine do Chastang.

\section{2" Usine di: Chastang}

L'évacuation des crues de l'usine-barrage de Chastang sur la Dordogne est prove à l'aide de deux evacuateurs en saut de ski amenages sur le loit de lusine aceolece at barratise. Les doux pertuis sont disposés symálriquement par rapport a l'axe de l'usine; chacun d'aux est oblute par une vanne-secteur de $13,6 \times 9,4 \mathrm{~m}$ foncliomnanl en charge et placée a l'aval du seuil déversant. Sur toule sa hauleur, le pertuis a und largeur constante de $13.6 \mathrm{~m}$. Ie dispositif de dispersion de la veine liguide silue i lextremile aval de ehatue coussier comporte ane dent conlrali ol deux chanfreins lateraux. Chacun des diversoirs peut evacuer un débit maximum de $2.000 \mathrm{~m}: \% / \mathrm{s}$ (fig. 27).

\section{a) Vilesses moyennes dans les sections:}

las essais ont eté axecutés avec un debil évacué de $620 \mathrm{~m} \% / \mathrm{s}$. I.es profils en travers ont été relevés dans cing sections régulièrement réparlies sur la partic aval du coursier. Six sections avaient ite initialement próvues; mais l'une d'elles, celle d'amont, n'a pu elre exploitée en raison des fortes venues d'an aux postes do me- 


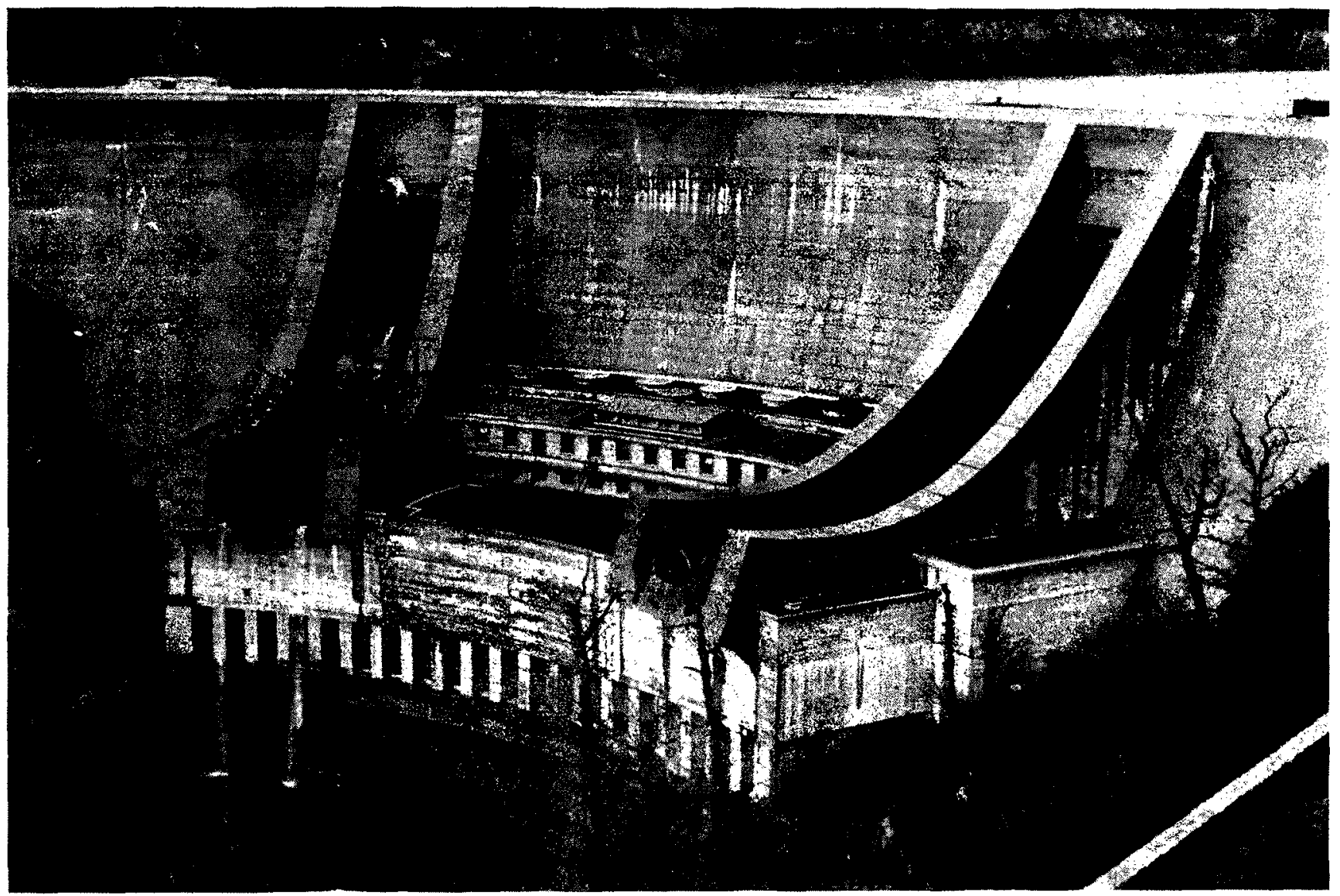

Fig. 27. - Evacuateurs de Chastang.

sure, dues à une fuite au joint superieur d'élanchéité de la vanne-secteur.

Le tableau ci-aprés résume les principaux résultats obtenus.

\begin{tabular}{|c|c|c|c|}
\hline Section & $\begin{array}{l}\text { Surface } \\
\text { mouillec } \\
\text { en } \mathrm{m}^{2}\end{array}$ & $\begin{array}{l}\text { Hatuteur } \\
\text { moyenne } \\
\text { en m }\end{array}$ & $\begin{array}{l}\text { Vitesse } \\
\text { moyenne } \\
\text { en } 111 / \mathrm{s}\end{array}$ \\
\hline $13 \ldots \ldots \ldots$ & 25,45 & 1,87 & 23,57 \\
\hline C.. & 25,35 & 1.86 & 23,67 \\
\hline 1) $\ldots \ldots \ldots$ & 25,32 & 1,86 & 23,70 \\
\hline E......... & 24,79 & 1,82 & 24,20 \\
\hline F.......... & 26,30 & 1,94 & 22,80 \\
\hline
\end{tabular}

On peut admetlre que les hauteurs, ainsi que les vitesses moyennes, sont déterminées à $\pm 2,5 \%$ près environ, sous réserve d'une estimation exacte du débit.

\section{b) Répartition des vitesses :}

x) Vitesses de surface. - Par suite des srandes vilesses d'écoulement (de l'ordre de $25 \mathrm{~m} / \mathrm{s}$ ), la dispersion des vitesses mesurées par différents flotteurs est relativement importante. Toutefois, la vitesse d'un même flotteur enregistrée sur les trois caméras a pu être déterminée à moins de $\pm 5 \%$ près.

Dans les trois sections d'observation des flotteurs, les valeurs encadrent la valeur théorique de la vilesse calculée, compte non tenu de la perte de charge $(\mathrm{V}=\sqrt{2 g \mathrm{H}}, \mathrm{H}$ étant la dénivelée entre le plan d'eau amont et la surface de la veine liquide dans la section considérée).

Ces vitesses restent comprises entre 27 et $28,5 \mathrm{~m} / \mathrm{s}$ dans les différentes sections de mesure.

3) Vitesses au voisinage du coursier. - $\mathrm{Au}$ cours de l'exécution des essais, nous avons relevé les niveaux des différents manomètres dès l'ouverture des vannes de l'évacuateur. Pour les faibles débits, nous avons constaté à chaque section une diminution progressive des pressions staliques. Pour le débit maximum de $620 \mathrm{~m}^{3} / \mathrm{s}$, les pressions statiques mesurées aux prises des lubes de Prrór à 5 et $15 \mathrm{~cm}$ du coursier étaient 
inférieures à la pression atmosphérique; un phénomène analogue avait dejà été constalé au cours du larage à l'air des tubes de Protor au Laboratoire d'Aérodynamique de Saint-Cyr; il peut s'expliquer par un dócollement de l'eau au droit des prises staliques des lubes de PIToT.

D'ailleur's, pour les faibles valeurs du débit, on a pu s'assurer que l'écart entre la pression statique réelle, déduite des prises de pression installées sur le radier et les prises statiques des tubes de PITot, concordait sensiblement avec celui obtenu en soufflerie pour la même vitesse.

Toulefois, cet écart plus important aux gros débits, rendait les corrections délicates. On a préféré évaluer la vilesse à partir de la différence entre la pression dynamique donnée par le tube de PrTo'T et la pression statique déduite des mesures sur le radier (tableau ci-contre).

L'erreur accidentelle sur la mesure des pressions dynamiques n'entraine pas une erreur supérieure à \pm 2 à $3 \%$ sur la valeur des vitesses, erreur à laquelle peut évidemment s'ajouter une légère erreur systématique due au mode d'évaluation de la pression statique. En outre, les difficultés expérimentales ont conduit pour l'une des prises $\left(n^{\circ} 4\right)$ à des résultats nettement aberrants.

\section{c) Mesure des pressions sur le radier.}

Par suite de l'obturation de quelques-unes des canalisations enrobées dans le béton, il n'a été possible de mesurer la pression qu'en trois points de l'évacuateur.

Ainsi qu'on peut le constater sur le lableau accompagnant la figure 28 , ces pressions coïnci-
VIILSSES AL VOLSINAGL DU RAIIER

\begin{tabular}{|c|c|c|c|c|}
\hline Prises & $\begin{array}{l}\text { Dislance } \\
\text { du Prrot au } \\
\text { radier en m }\end{array}$ & $\begin{array}{l}\text { Pression } \\
\text { dynamique } \\
\text { en m d'eau }\end{array}$ & $\begin{array}{c}\text { Pression } \\
\text { statique } \\
\text { en in d'eau } \\
(")\end{array}$ & $\begin{array}{l}\text { Vifesso } \\
\text { ell } \mathrm{m} / \mathrm{s}\end{array}$ \\
\hline \multirow[t]{2}{*}{1} & 0,05 & 21,66 & 5,0 & 18 \\
\hline & 0,15 & 21,08 & 1,7 & 19.5 \\
\hline \multirow[t]{2}{*}{2} & 0,05 & 24,17 & 5,0 & 19,6 \\
\hline & 0,15 & 28,48 & 1,7 & 21,6 \\
\hline \multirow[t]{2}{*}{4} & 0,05 & - & - & $-\cdots$ \\
\hline & 0,15 & 15,12 & 4,8 & $1.1,2$ \\
\hline \multirow[t]{2}{*}{5} & 0,05 & 23,96 & 4,35 & 19,6 \\
\hline & 0,15 & 25,16 & 4,2 & 20,2 \\
\hline
\end{tabular}

(*) Valeur déduile des mesures sur le radier.

dent d'une manière très salisfaisante avec celles déduites des essais sur modele réduil au Laboratoire National d'Hydraulique.

Ces mesures plus completes effectuées a Chastang ont apporté des éléments précieux, comme on le verra ci-apres, pour la détermination des pertes de charges en facilitant l'évaluation de certaines corrections.

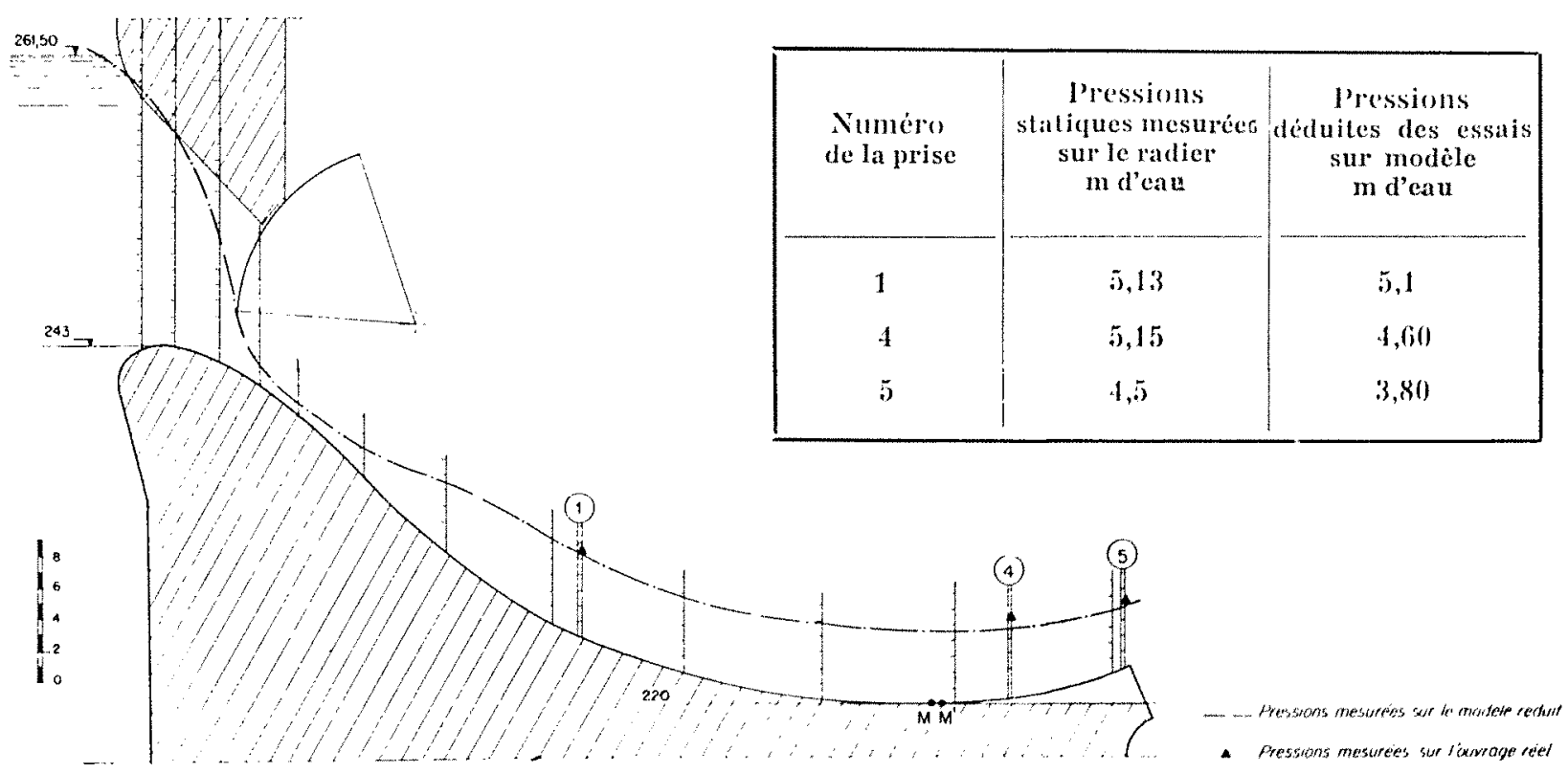

Fig. 28. - Evacuateur de Chastang. Pressions sur le radier $\left(Q=630 \mathrm{~m}^{3} / \mathrm{s}\right)$, 


\section{C) Détermination de la ligne de charge.}

Pour determiner la valeur de la charge totale dans une seclion à partir de sa définition :

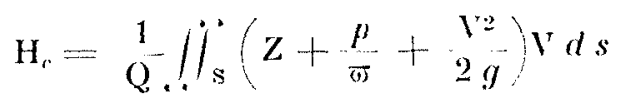

le plus simple est de meltre en évidence les différents termes composant la charge.

La relation ci-dessus s'écrit, en décomposant la pression en pression statique $p_{0}$ el en pression dynamique $p_{1}$, due à la courbure (of. figs. 30 ) :

$$
\begin{aligned}
& \mathrm{H}_{c}=\frac{1}{Q} \|_{\mathrm{s}}\left(\mathrm{Z}+\frac{p_{0}}{\bar{\omega}}\right) \mathrm{V} d \mathrm{~s} \\
& +\frac{1}{Q} \iint_{\mathrm{s}} \frac{p_{1}}{\sigma} \mathrm{\sigma} d s+\frac{1}{\mathrm{Q}}-1 \int_{\mathrm{s}} \frac{\mathrm{V}}{2 g} d s
\end{aligned}
$$

Soit en notant que l'on a dans une section donnée:

$$
\begin{gathered}
Z+\frac{p_{0}}{\sigma}=p_{0}^{*}=\mathrm{C}^{\mathrm{t}} \\
\mathrm{H}_{c}=p_{0}^{*}+\frac{1}{\mathrm{Q}} \iint_{\mathrm{s}}^{*} \frac{p_{1}}{\sigma} V d s+\nu_{c} \frac{\mathrm{V}_{m}^{\prime \prime}}{2 !}
\end{gathered}
$$

Le calcul de la charge nécessite done :

1) Ia connaissance de la pression dynamique;

2) La connaissance de la valeur vraie de la vitesse moyenne, compte tenu de l'existence des zones latérales émulsionnées;

3. La connaissance du coefficient $x_{a}=\frac{\iint_{s} V^{\prime a S}}{Q_{0} V_{m}{ }^{2}}$

a) Calcul du terme relalif a la pression dynamique :

Nous supposerons la pression constante à unc cule donnée dans toule la seclion.

Lat fression dynamique sécrit, en un point queleonyue de la section :

$$
\frac{p_{1}}{\pi}=\int_{r_{0}}^{r} \frac{V: 2}{g r} d r
$$

arec:

$r$ : rayon de courbure de la trajectoire de la particule dont la vilesse est $\mathrm{V}$;

$r_{1}$ : rayon de courbure de la surface libre.

Il est possible d'en calculer la valeur si l'on comnaît la répartilion des vilesses. I a eonsideration de l'écoulement hélérogiene (figs. 30), conche limite et noyau, compliquant inutilement le calcul, il est plus simple d'encadres la vaie valeur de celte pression dynamique en supposant

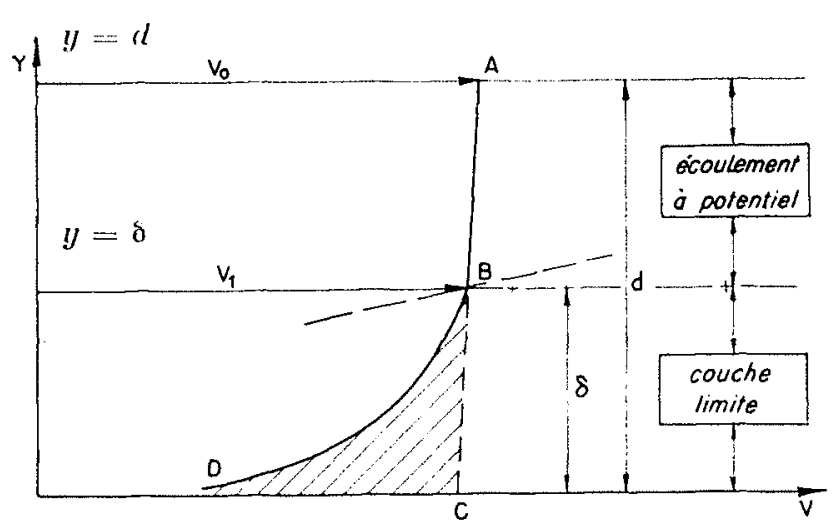

lia. 29. - Ecoulement hétérogène sur badier curviligne: schema de la distribution des vitesses.

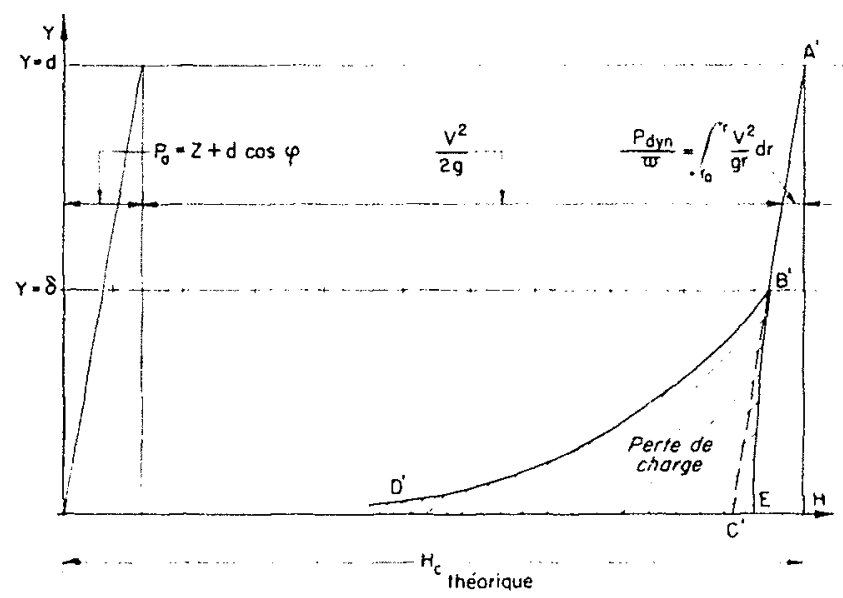

Fli, 30. - Ecoulement héderogène sur radier curviligne: distribution des composants de la charge d'un filet liquide.

que l'úpaisseur de la couche limite est nulle, ou ou contraire égale au tirant d'eau.

On montre, d'autre part, que dans los deux ras, le lerme :

$$
\frac{1}{Q} \iint_{s} \frac{p_{1}}{\pi} \mathrm{V} d s
$$

qui represente la valeur moyenne de la pression dymamique dans la section, dilfere tres peu de la demi-pression dynamique sur le radier :

$$
1 / 2\left(p_{1} / \pi\right)_{\text {radier }}
$$

La recherche d'une methode de calcul phus précise de la pression dynamique sur lo radier est rendue inutile du fait que ces cvaluations difrient systématiquement par défrunt des mestures expérimentales. L'écart observé à Chaslang, comme il l'avait été sur le modile de Bort, alteint selon les cas 15 à $20 \%$ de la pression totale mesurec sur le radier, e'est-à-dire 30 a $40 \%$ de la valeur de la pression dynamique lorsque celle-ci est du même ordre de grandeur que la pression statique.

Il semblerait que cel ceart soil inhérent a la mélhode de mesure par prises de pression sur 
radier curviligne, mais il est difficile d'affirmer quelle est la rraie grandeur de la pression au fond.

Devant cette divergence, et compte lenu de la faible importance du terme relatif à la pression dynamique par rapport à la charge, nous avons adoplé la valeur moyenne des deux modes de calcul (avec ou sans couche linite) dont les résultats different d'ailleurs de moins de $15 \%$. On verra plus loin l'intérèl de cette évalualion probablement approchée par défaut.

\section{b) Evaluation de la vitesse molyenne vraie :}

L'erreur sur la vitesse moyenne $V_{m}=Q / S$ est la somme des erreurs résultant de l'ereur sur le débit d'une part el de l'erreur sur la section mouillèe d'autre part.

Le débit a ćté mesuré, on l'a ru, arec une erreur maxinum $d \mathrm{Q} / \mathrm{Q}= \pm+\%$. Il en résulte sur l'énergie cinélique une erreur igale à :

$$
\frac{d V_{m}^{2}}{V_{m}{ }^{2}}= \pm 8 \%
$$

La section monillée relevée comprend la section occupée par l'air contenu dans les deux couches latérales émulsionnées dont on ignore lépaisseur. Il en résulte une creur systémati- que sur la section récllement occupéc par lícoulement, erreur dont on peut diffieilement déterminer la valeur la plus probable, mais dont il est possible d'évaluer un minimum.

A Chastang, les couches émulsiomnés laterales ont $2 \mathrm{~m}$ de large. Supposons que l'émulsion contienne $50 \%$ d'air el que la profondeur de celte couche soit seulement de $0,50 \mathrm{~m}$.

La section oecupé par l'air est de 1 m". Ia variation relative de la section monillec esl done:

$$
\frac{d s}{s}=\cdots+i
$$

Soit :

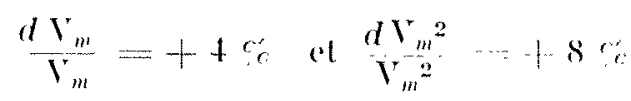

On voil l'importance de celte correction, dont la valeur valic est sans doule superieure a liesm limation ci-dessus.

D'autre part, le sens de celle correction jourant dans le sens d'une majoration de la charge, on wil qu'il est plus aisé de determiner une valeur minimum de colle-ei que sa valeur moyenne la plus probable.

\begin{tabular}{|c|c|c|c|c|c|c|c|c|}
\hline SECTIONS & $\begin{array}{l}1 \\
\vdots \\
1\end{array}$ & & C. & $!$ & 1) & 8 & $1:$ & 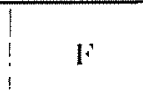 \\
\hline Cote radier. . . . . . . . . & 222,7 & & 221 & & 220.15 & & 20 & 1200.20 \\
\hline Pression statique $d \cos \varphi \ldots \ldots$ & 1,75 & & 1,80 & 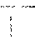 & 1,85 & $!$ & $1, x !$ & $1,(3)$ \\
\hline Pression dynamique $1 / 2\left(p_{1} / \pi\right)_{\text {rarier }} \ldots$ & 1,30 & & 1,25 & 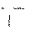 & 1,15 & & 0 & 1 \\
\hline Cote ligne piezometrique............. & 225,75 & & 224.05 & : & 20.3 .0 .5 & & 221,82 & 223.10 \\
\hline$v_{m}^{2 / 2}$ ! mesure $x_{n}=1 \ldots \ldots \ldots$ & 30,20 & & 30,50 & & 30.60 & & 31.83 & 28,25 \\
\hline 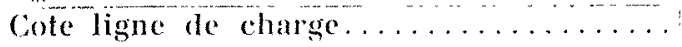 & $25,5,95$ & & $251,5 \%$ & & 253,65 & & 253.635 & 251.35 \\
\hline Perte de charge .......... & 5,55 & & 0,95 & & 7.85 & & 7.85 & 10,15 \\
\hline
\end{tabular}

EVACUATELR DE CHASTANG

$Q=620 \mathrm{~m}^{3} / \mathrm{s}$. - Retenue amont : 261,50

\begin{tabular}{|c|c|c|c|c|c|}
\hline SECTIONS & $\begin{array}{l}F \quad F \quad E \\
|x=10,10| x=18,36\end{array}$ & $\begin{array}{c}\mathrm{D} \\
x=32,76\end{array}$ & $\left\{\begin{array}{l}c \\
x=10,3610\end{array}\right.$ & $\begin{array}{c}13 \\
x=50,40\end{array}$ & 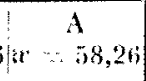 \\
\hline 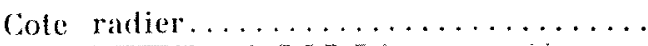 & $503,60: 492,30$ & 482,65 & 180.90 & 180,20 & 180,20 \\
\hline Pression statique $d \cos \varphi$. & $1,10: \quad 0,70$ & $1,0(0$ & 1,10 & 1,20 & 1,60 \\
\hline Pression dynamique $1 / 2(p / / \pi)_{\text {radigr }} \ldots \ldots$ & $-0,10 \quad 0,40$ & $(0,70)^{\cdots}$ & 0,751 & 0 & 0 \\
\hline Cote ligne piézométrique.............. & $514,3 0 \longdiv { 4 9 3 , 4 0 }$ & 484,35 & 482,75 & 181,10 & 181.80 \\
\hline$V_{m}{ }^{2 / 2} g$ mesuré $\alpha_{c}=1 \ldots \ldots \ldots \ldots \ldots$ & $7,65,18,30$ & 22,90 & 22,90 & 21.20 & 19,10 \\
\hline Cote ligne de charge ................ & $511,95,511,70$ & 507,25 & 505,65 & 502,60 & 501,20 \\
\hline Perte de charge...... & 2,80 & 7.25 & 8.851 & 11,90 & 13,30 \\
\hline
\end{tabular}

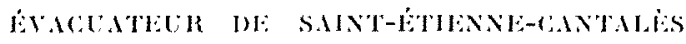

$Q=230 \mathrm{~m} \% / \mathrm{s} .-$ lefenue amont : 514,50 


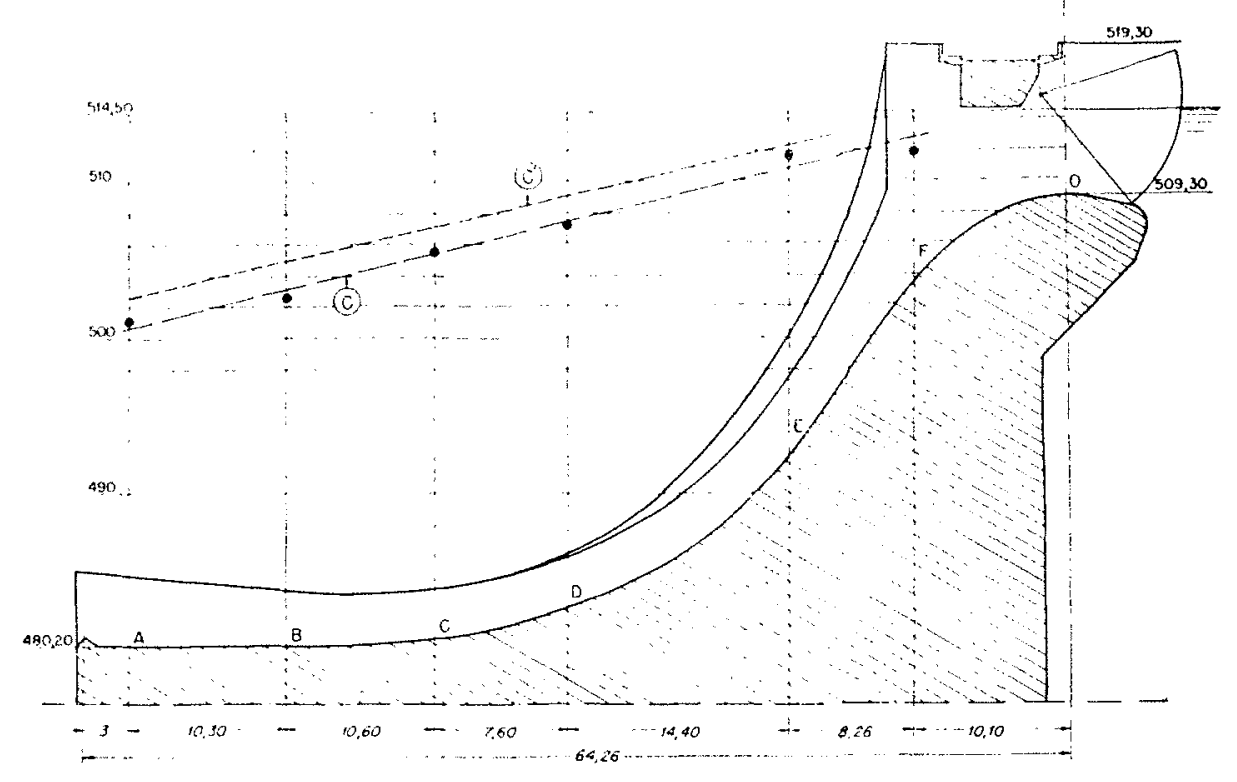

Fili. 31.

devaci:ateun

DE

SANT-ETHNNE-CANTALÈS.

Ligne de charge pour un deversement de $230 \mathrm{~m}^{3} / \mathrm{s}$.
FIG. 32 .

Evaceateur rive proite DF Chastang:

Ligne de charge pour un deversement de $620 \mathrm{~m}^{3} / \mathrm{s}$.

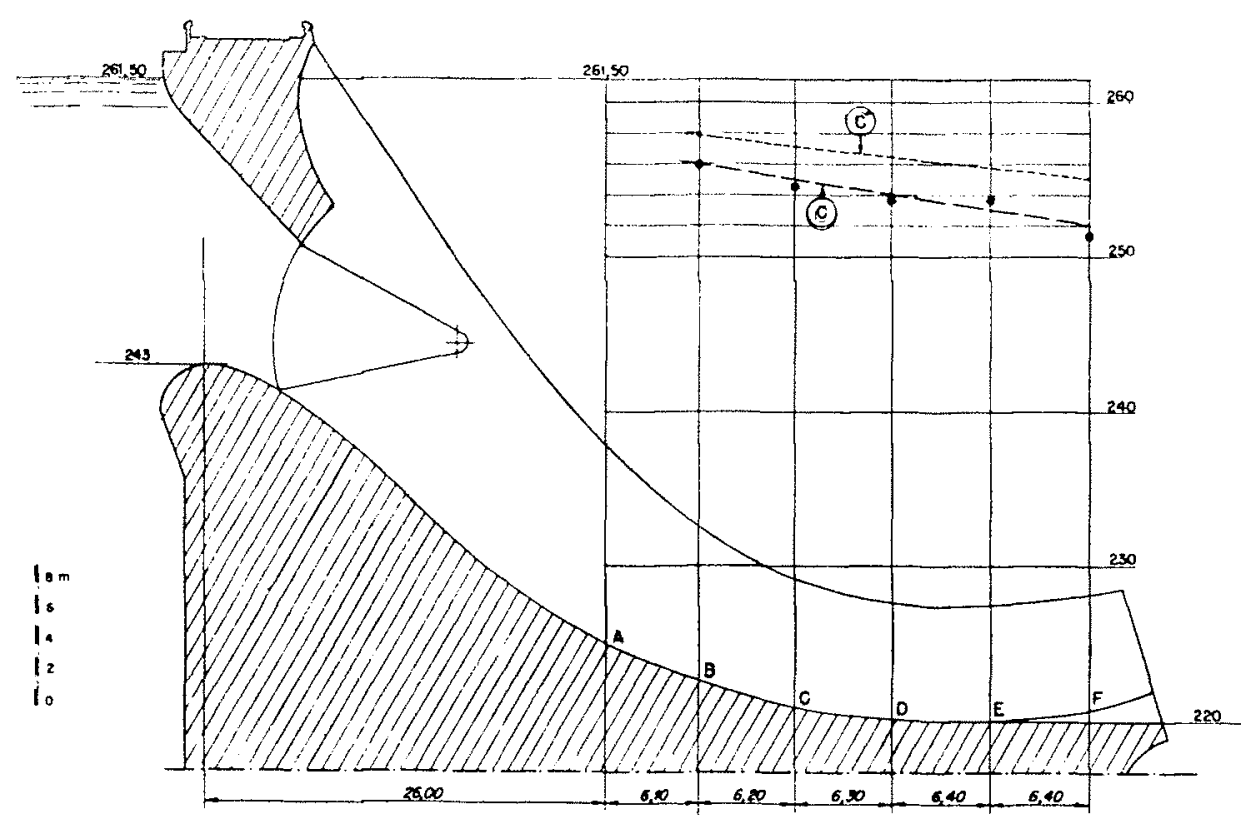

c) Coefficient $x_{c}$ de répartition des vitesses:

La nature de l'écoulement étanl connue, ainsi que l'épaisseur de la couche limite, il est possible, en principe, d'évaluer $\alpha_{c}$ pour le noyau d'une part, pour la couche limite d'autre part, le coefficient résullant étant donné par la relation :

$$
z_{c}=\frac{\alpha_{c} \mathrm{~N} \cdot \mathrm{Q}_{\mathrm{N}} \cdot \mathrm{V}_{\mathrm{N}}{ }^{2}+\alpha_{c \mathrm{~L}} \cdot \mathrm{Q}_{\mathrm{L}} \cdot \mathrm{V}_{\mathrm{L}}^{2}}{\mathrm{Q} \cdot \mathrm{V}_{m}^{2}}
$$

arec :

$x_{c N}, Q_{N}, V_{N}=$ quantités relatives au noyau, $a_{c} \mathrm{~L}, \mathrm{Q}_{\mathrm{L}}, \mathrm{V}_{\mathrm{L}}==$ quanlités relatives à lat couche $\mathrm{l}$ mite.

On peut négliger $\alpha_{c} \mathrm{~N}$ qui est pratiquement égal à l'unité. Mais on rencontre, dans lévaluation de $\alpha_{\varphi}$, l'incertilude qui pesait déjà sur le calcul de l'épaisseur de la couche linite el corrélativement sur la répartition de vitesse à l'intérieur de celle-ci.

Aussi convient-il de remarquer :

- Que le sers de la correction apportéc par $\alpha_{c}$ esl connu puisque $\alpha_{c}>1$; 
Que limportance de la correction ipportee par $x_{c}$ est inferieure à celle apporté par l'émulsion d'air puisque les diverses évaluations approximatives que l'on peul faire conduisent a des valeurs comprises entre 1,02 et 1,05 . Il est done inutile de rechercher une évaluation plus precise.

De ces diverses corrections, on peul conclure :

1. Que les corrections dont on connait le sens tendent à majorer la charge de $10 \%$ environ et sont au moins égales a l'erreur systémalique de sens inconnu pouranl résulter d'une erreur sur le délbil $(-2.8 \%)$.

2. - Qu'une évaluation plus précise de certaines corrections serait illusoire. La ligne do charge résultant des lectures brutes $\left(z_{\%}=1\right.$, seclion occupe par l'air supposée nulle) donne done un maximum de la perte de charge. La ligne de charge la plus probable correspondant à une majoration de $10 \%$ de l'únergie cinétique se situerail $3 \mathrm{~m}$ plus haut environ (lig. 32, Courbe $\mathrm{C}^{\prime}$ ).

On a représente de mòme la ligne d'énergie diduite des mestures do Saint-Elienne-Cantales, ansi que la mème correction de $10 \%$ de l'encrsie cinétique (fig. 31). Notons que, pour cel ouvage, la correction due a la zone supericielle cmulsionne est probablement plus imporlante dans la parlic aval que dans le cas précédent du fait de la veine refléche provoquée par la convergence du bajoyer Rive droile (fig. 5). En oulre, celte correction croit certainement de lamont vers l'aval. Par contre, celte corretion systémalique du lerme cinétique a une incidenec moindre en valeur relalive sur la perte de charge, da fail de la valeur plus importanle de colle-ci.

\section{TROISI È M E P A R T I E}

\section{A) Le développement de la couche limite sur le radier des évacuateurs de surface : comparaison des résultats théoriques et expérimentaux}

Il a paru intéressant d'appliquer à l'évacuateur de Chastang la mélhode de calcul proposce par M. Hacbnown (*), en partant des résultals des mesures faites sur louvrage réel. Ce travail a été fait par M. GODIN, ingénieur ćleve des Ponts et Chaussces, que nous remercions de sa participation active à celte partie de l'étude.

Cette vérification avait l'intérèt de compléter celles déjà faites par M. Halbrons à partir d'essais à l'échelle réduile réalisés, soit à Grenoble, soit à Chatou. En outre, la coïncidence du calcul et de l'expérience devait permettre une exploitation plus sûre des résultats de l'expérience, en ce qui concerne la perte de charge sur louvrage dont nous parlerons plus loin.

\section{PRINCIPE DE LA MÉTHODE DE CALCTL THÉORIQUT:}

Rappelons que l'évaluation théorique proposce par M. Harbrons part des hases suivantes:

a) Relation intégrale de Von Karmas, donnant

\footnotetext{
(*) G. Habrons. - Etude de la mise en légime des econlements sur les ouvrages a torte pente. La Homalle Blanche, $\mathrm{n}^{\prime 4} 1,3$ at 5,1952 .
}

la valeur de la vitesse de frollement $V_{*}$ à parlir de la répartilion de vilesse dans la couche limile et de la loi de variation de la vitesse $V_{1}$, an bord de la conche limite en fonclion de l'abscisse.

$V_{*}^{2}=\frac{d V^{r}}{d x} \int_{0}^{-\delta}\left(V_{1} \cdot V\right) d y+\underset{d x}{d} \int_{0}^{0} V\left(V_{1} \ldots V\right) d !$

b) Hypothese : Répartition des vilesses dans la couche limile identique a la répartition logarithmique elassique en régime blabli el qui s'ecrit en regime rugueux :

$$
\frac{V^{r}}{V_{*}}=8.48+5,75 \log \frac{y}{\varepsilon}
$$

Au moyen de ces deux relations, on peul évaluer dans une section queleonque (fig. 29) les deux ineomuces $V_{*}$ et $\partial$ à condition de connable:

$$
\begin{aligned}
& Y_{1}=\text { vitesse dans le noyau au conlact de la } \\
& \text { couche limite; } \\
& \varepsilon=\text { rugosité éfuivalente. }
\end{aligned}
$$

En pratique, M. Hankox choisit comme inconnues : $Z=\frac{V_{1}}{V_{*} / k}$ at i. 11 est donc nécessaire 
de connaitre les valeurs de $V_{1}$ et $\varepsilon$ pour pouvoir appliquer celte méthode de calcul.

Examinons ces deux points suceessivement:

\section{x) Vilesse $V_{1}$ dans le noyau :}

La vitesse $V_{1}$ se détermine aisément à partir des deux relations fondamentales de l'écoulement en énergie constante sur rardier curviligne, les filets liquides atant supposés concentriques dans une seclion donnée $\left.{ }^{*}\right)$.

On a dans toute la misse de l'écoulement:

$$
Z+\frac{\pi}{p}+\frac{Y 2}{2 g}=\mathrm{C}^{\mathrm{H}}
$$

d dans une section droite :

$$
\text { V. } r_{0}=C^{\prime \cdot}
$$

$r=$ rayon de courbure de la trajectoire de la particule dont la vitesse est $r$.

\section{3) Rugosite équibalente $\varepsilon$ :}

Ce n'est qu'ì titre de grossiere ajproximalion que l'on peut prendre une evaluation géométrique de la valeur de $\varepsilon$ d’après les irrégularités du radier.

Nous avons donc adopté, comme M. Halbnows l'avait fait pour les résultats obtenus sur le modele au 1/60 de l'évacuateur de Bont, une míthode qui consiste à ne eonsiderer \& que comme un intermédiare de calcul, que l'on évalue à partir d'une donnéc expérimentale: li: position du point eritique à un certain débit.

Connaissant $\varepsilon$, on peut calculer l'épaisseur de la couche limite s en une section queleonque de l'ivacuateur. En effet, o et $z$ sont fonction de la vilesse $V_{1}$ dans le noyau, c'est-à-dire de la charge $H$, de labseisse ste long du radier, el de la rugosité éfuivalente $\varepsilon$; par contre, clle est indépendante du débit, si ce n'est pas l'intermèdiaire de $\mathrm{r}_{1}$, qui varie un peu avec le tirant d'eau.

A partir de ò el de $z$, la répartition de vitesse est donnce dans loute l'épaisseur de l'écoulement par les relations (2) et (4), applicables respectivement dans la couche limile ot dans le novau, a condition, loutefois, de connaitre le tirant d'cau lotal $d$.

\footnotetext{
(-) Cf. R. Martre. - Note sur le fonetionnement des ivaruateurs de surface aux ouvertures partielles de
} vamme. La Houille Blanche, $\mathrm{n}^{\circ} 2,1952$.

\section{$2^{\circ}$ Application a L'Évacuateur of: Chastrang}

Le calcul de la rugosité équivalente a été fait, conformément à ce qui précède, à partir du relevé de la position du point critique au débit de $250 \mathrm{~m}^{3} / \mathrm{s}$. On a estimé d'après les photographies (cf. planche II) que le point critique se trouvait atu point has du coursier à ce débit.

On obtient après calculs : $\varepsilon=0,5 \mathrm{~mm}$ environ; valeur qui n'a qu'une signification indicative puisque, pour une variation du débit d'affleurement de $\pm 10 \%$, $\approx$ passe de $0,22 \mathrm{~mm}$ à $1,25 \mathrm{~mm}$; cette imprécision ne présente cependant pas d'inconvénient grave, $\varepsilon$ n'étant qu'un intermédiaire de calcul.

Les mesures expérimentales ayant été réalisées pour un seul débit de $620 \mathrm{~m}^{3} / \mathrm{s}$, la vérification proprement dile a consisté à déterminer dans quatre sections de mesure, à partir de la valeur du tirant d'eau moyen mesuré, la répartition de vitesses, d'où on déduit le débit par mètre de largeur, puis le débit. Notons que tous ces calculs ont été faits en adoptant une largeur fictive du coursier inférieure à sa largeur réelle et déterminée de manière à tenir compte des couches limites latérales à partir de l'estimation de l'épaisseur de ces couches sur les photos de l'écoulement. Pour l'essai à $620 \mathrm{~m}^{3} / \mathrm{s}$, cette largeur fictive est de $13,10 \mathrm{~m}$ (largeur réelle $13,50 \mathrm{~m}$ ).

Le calcul complet conduit aux résultats sui-

\begin{tabular}{|c|c|c|c|c|}
\hline Sections & $B$ & C: & D) & : \\
\hline$==\frac{V_{1}}{V_{*} / k}$ & 10,36 & 10,40 & 10.58 & 10,70 \\
\hline$\delta$ & $0,54 \mathrm{~m}$ & $0,61 \mathrm{~m}$ & $0,69 \mathrm{~m}$ & $0,76 \mathrm{~m}$ \\
\hline$Q$ & $\left(228 \mathrm{~m}^{2} / \mathrm{s}\right.$ & $637 \mathrm{~m} \% / \mathrm{s}$ & $613 \mathrm{~ms} / \mathrm{s}$ & $638 \mathrm{~m}^{3} / \mathrm{s}$ \\
\hline
\end{tabular}
vants :

Le débit mesuré au limnigraphe d'Argentat el contròlé par jaugeage étant de $620 \mathrm{~m} \% / \mathrm{s} \grave{a} \pm 4 \%$ près, ces résultats sont parfaitement cohćrents, d'autant plus que si l'on excepte le point E, la croissance du débil calcule de $B$ en $D$ peut s'expliquer par l'augmentation du pourcentage d'air contriiné dans les couches limites latérales, d'amont ver's l'aval. Notons d'ailleurs que si l'on suppose l'écoulement entièrement à potentiel, hormis les couches limites latérales, le débit dans la section E s'écrit :

$$
\mathrm{Q}_{1}=1,82 \times 13,1 \times 27,9=665 \mathrm{~m} 3 / \mathrm{s}
$$


L'écart est donc supérieur aux erreur's de mesure. Par contre, si on suppose la valeur de $\delta$ lixe et égale à sa valeur en $\mathrm{E}, \hat{o}_{1:}=0,76 \mathrm{~m}$, on trouve dans les différentes sections des débits qui restent compris entre 620 et $640 \mathrm{~m}^{3} / \mathrm{s}$, c'est-a-dire à l'intéricur du domaine défini par la précision des mesures $\left( \pm 20\right.$ à $25 \mathrm{~m}^{3} / \mathrm{s}$.)

On voit donc que si les mesures permettent d'affirmer l'existence d'une couche limite, clles ne permettent pas de vérifier si la Joi de variation resutant de la méthode de cateul de M. HatBRoxx est préférable à une autre.

Conme, d'autre part, il est nécessaire de mesurer l'épaisseur de la couche limite à un certain débit pour évaluer $\varepsilon$, on peut se demander si cette méthode d'évaluation de mise en œuvre assez longue est susceptible d'etre utilisée en pratique pour la détermination a priori des conditions d'écoulement sur un ouvrage.

Notons, enfin, qu'on a comparé dans la seclion B la répartition de vitesse calculée avec les deux valeurs mesurées (fig. 33).

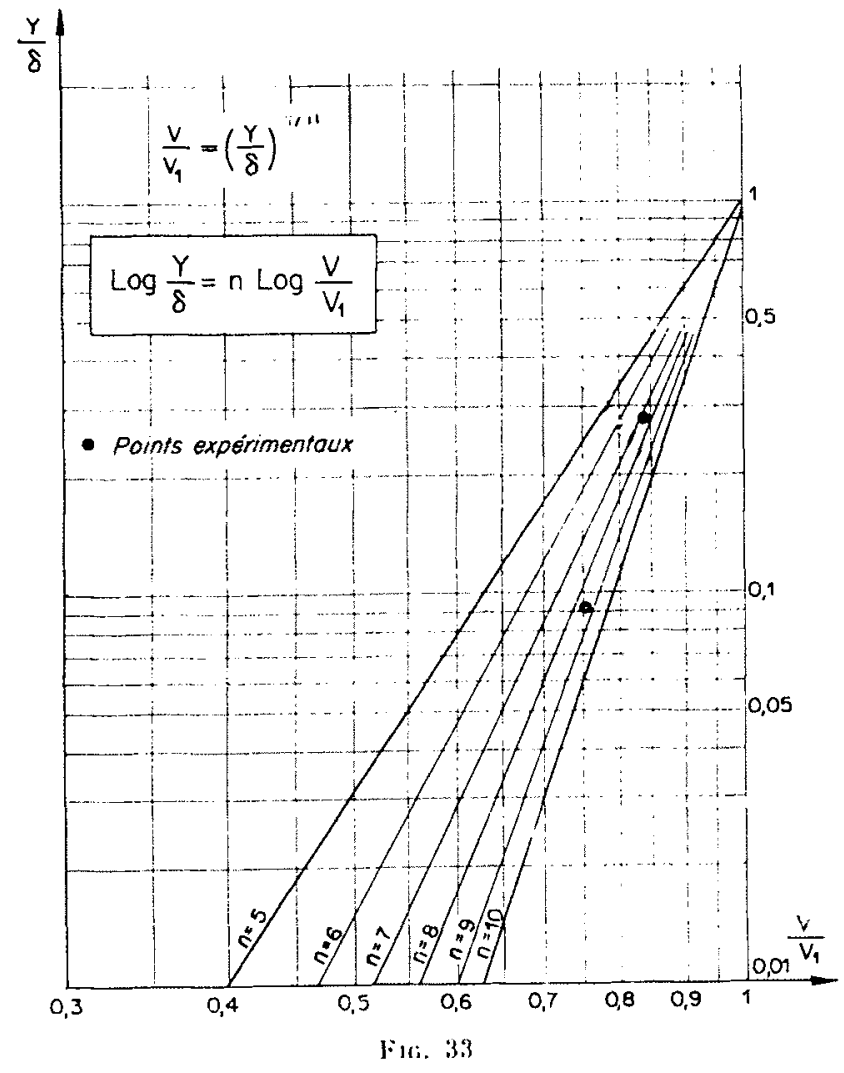

Cette comparaison a efe faite en utilisant la loi de vitesse approchée :

$$
\frac{v_{1}}{r_{1}}=\left(\frac{y}{\delta}\right)^{1 / n}
$$

Cetle loi differe peu de la loi logarithmique ef les calculs en sont simplifies. En considerant la ritesse moremne $V_{m}=V_{1}\{n /(n+1)\}$, on montre aisciment que l'on a :

$$
n=Z--1
$$

Soit, pour $Z=10,3, \quad n=9,3$ environ. I. A's points experimentaux seraient plus roisins de la courbe $n=8$. Toulefois, chant donné la précision de's mesures de vitesse, on ne peut eonclure ì une divergenee nette.

\section{3" Conciteshoxs}

Cette confrontation des mithodes de calcul thioriques et de lexperience ne permet d'intismer aucune des hypothises de depart : existence des vilesses d'écoulement thériques dans ke noyau a potenticl, et diune couche limite turbulente a distribution de vitesses legarithmique.

En dehors des phémomenes d'emulsion, les ceoulements à grande vilesse obéissent done, semble-t-jl, aux lois chassiques de l'hydraulique.

Il n'existe pas datutre vilesse limite que celle résultant de lapplieation de la loi de Braxoctatas au noyau a potentiel, ce qui conduit pour le filet de surface à la vitesse theorique $V_{0}=V^{2} g \mathrm{ll}$, déjà mentionnéc plus hatul; les quelques mesures de vilesse de surface par llotteurs róalisés a Chastang confirment dailleurs ae resultat.

La similitude de ces exoulements a grande vitesse est tout a l'at valable, en particulier pour les débits importants oì l'émulsion intervient trés peu. Lat coñncidence dess répatititions dess pressions sur lonvrage el sur le modele te confirme sur un point parliculier.

La seule diffieullé en ce qui concerne la similitude est de rétiliser correctement des rugosilis semblables, chant donne l'influenec importante de la valeur de e sur l'éprisseur de ba couche limite. Nous avons resolu le probleme dans te cas de l'etude sur modile de l'évactateur de Bort an laboraloire on roblisant suceessiventent deux rugositis differentes encadranl la valeur probable de la rugosile.

\section{B) Les pertes de charge sur le coursier des évacuateurs de surface.}

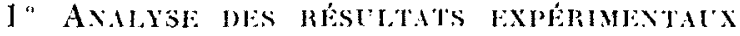

Elant donne la precision des mesures obtenue lors des essiris de Saint-Etienne-Cintalies ol de
Chastang, il serait illusoire de chercher at determiner le cocfirient des pertes de charge de section en section. En effet, lit perte de charge entre deux sections consecutives est on sinneral in 
mène ordre de grandeur que l'erreur possible sur la perte de charge en un point.

Notons, simplement que si l'on représente la perte de charge en fonction de l'abseisse curviligne sur le radier, ce qui a été fait pour les résultats obtenus à Saint-Etienne-Cantalès, il apparait que la perte de charge cumulée croit comme une fonction puissance dexposant supéricur à l'unité en fonction de l'abscisse curviligne, ce qui est normal, la perte de charge élémentaire devant crôtre de l'amont vers l'aval du lait de l'augmentation des vitesses moyennes d'une part, du développement de la couche limile d'autre part. Mais la dispersion des mesures est trop grande pour qu'un résultat quantitatif valable puisse en être déduit.

Il est seulement possible de déterminer un coefficient de pertes de charge global pour l'ouvarage. Ce peut être un procédé de calcul commode, mais la signification physique d'un tel coefficient peul ètre contestée étant donné la nature hétérogine de l'écoulement. La perte de charge est en eflet concentrée dans la couche limite.

Nous considererons simplement la perte de charge globale en prenant une valeur située dans l'intervalle défini par les courbes $\left(C_{i}\right)$ et $\left(C^{\prime}\right)$, à savoir :

\begin{tabular}{|l|c|c|c|c|c|}
\hline & $\begin{array}{c}\text { Perte } \\
\text { de charge } \\
\Delta \mathrm{H}\end{array}$ & $\Delta \mathrm{H} / h_{0}$ & $h_{0} / \mathrm{H}_{0}$ & \lrcorner $\mathrm{H} / \mathrm{H}_{0}$ & $\mathrm{~L} / \mathrm{d}$ \\
\hline Chastang........ & $8 \mathrm{~m}$ & $20 \%$ & 0,60 & $12 \%$ & 40 \\
St-Etienne-Cantales & $12 \mathrm{~m}$ & $35 \%$ & 0,55 & $19 \%$ & 60 \\
\hline
\end{tabular}

avec :

$h_{0}$ : chute sur l'ourrage lui-même,

$\mathrm{H}_{0}$ : chute totale,

$\Delta H$ : perte de charge.

Ces valeurs de la perte de charge sont sensiblement inférieures aux valeurs qui résultaient d'essais réalisés il y a quelques années au barrage de Laigle, pour un débit correspondant à une valeur de $L / d$ très voisine de celle de SaintEtienne-Cantalès.

L'explication réside, à notre avis, dans le fait que les expérimentateurs n'avaient pu, faute d'autres moyens de mesure, que relever les tirants d'eau au voisinage du bajoyer dans la zone où se produit un gonflement local du fait de la couche émulsionnée.

Or, une erreur de $10 \%$ par excès, sur la section conduit, on l'a vu, à une erreur de $20 \%$ par défaut sur la valeur de la charge, non com- pris les corrections dont nous avons parlé plus haut.

On note, par contre, une concordance correcte entre les mesures réalisées à Saint-Etienne-Cantalès et celles réalisées sur le modèle réduit au

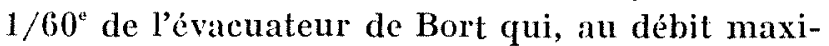
mum de $1.200 \mathrm{~m}^{3} / \mathrm{s}$, a une longueur relative $\mathrm{L} / d=60$, Le tirant d'eau aval a été mesuré pour trois rugosités différentes du radier. Etant donné la précision des mesures, on a confondu la charge avec la valeur du lerme cinétique el on a supposé $\alpha_{c}=1$.

Les resultats obtenus sur le modele pour le débit de $1.200 \mathrm{~m}^{3} / \mathrm{s}$ figurent ci-après :

\begin{tabular}{|c|c|c|c|}
\hline $\begin{array}{c}\text { Nature } \\
\text { du radier } \\
\text { du modèle réduit }\end{array}$ & $\mathrm{V}_{m} / \mathrm{V}_{0}$ & $V_{m}{ }^{2} / V_{6}$ & $\Delta \mathrm{II} / h_{\mathrm{t}}$ \\
\hline Vernis .. & 0,84 & $0,1,7$ & $29 \%$ \\
\hline Ciment lissé. & 0.82 & 0,71 & $33 \%$ \\
\hline$\dot{s} a b l c \quad \varnothing=2 \mathrm{~mm} \ldots$ & 0,69 & 0,18 & [ים \\
\hline
\end{tabular}

avee:

$$
\begin{aligned}
& \mathrm{V}_{m}=\text { vitesse moyenne mesurée. } \\
& \mathrm{V}_{0}=\text { vitesse thérique } \sqrt{2 g h_{0}} .
\end{aligned}
$$

Si l'on tienl comple de la correspondance entre les rugosités modile et réel, telle que l'on peut l'évaluer au moyen des coefficients de Manning par exemple, on voit que le vernis et le ciment lissé correspondant approximativement à du béton à état de surface normal d'une part, à du béton en mauvais état d'autre part. Or, l'ouvrage de Saint-Etienne-Cantalès, réalisé pendant la guerre, porte des traces de cofrrage très apparentes, ce qui explique le chiffre $\Delta \mathrm{H} / h_{0}=35 \%$ trouvé ci-dessus.

Ces quelques résultats, obtenus sur modèle ou sur ouvrages réels, semblent confirmer l'intérêt du classement des ouvrages en fonction de leur longueur relative.

On a cherché à évaluer quelle serait la perte de charge à Chastang au débit critique $Q \# 270 \mathrm{~m}^{3} / \mathrm{s}$ qui correspond à une longueur relative $\mathrm{L} / \boldsymbol{d}=100$. On a admis pour cela que la quantité d'énergie dissipée reste constante au-delà du débit critique, en la corrigeant toutefois dans le rapport des périmètres mouillés qui est le 1,15.

La perte de charge relative serait donc au débit critique :

$$
\left(\frac{\Delta H}{h_{0}}\right)=20 \% \times \frac{1}{1,15} \times \frac{620}{270}=40 \%
$$

Cette évaluation montre qu'il est très normal de relever des pertes de charge approchant de 
$50 \%$ lorsqu'on se place dans la zone des fabibles déhits.

Rappelons entin qu'on at souvent lenté, dans le cas des ouvrages en saut de ski, de délerminer la perle de charge au moyen de la mesure de la porté du jet en supposant valable la trajectoire parabolique de chule libre, ce qui neglize le frottement dans l'air.

Ies résultats obtenus par cette méthode nous semblent assez sujels a caution dams lo als des ouvrages réels do fait des nombreases difficulles auxquelles se heurlent les mesures:

Dillicultés d'observalion du fait des embrums el des projections;

- Difficultés dues aux dispositifs de dispersion qui rendent plus délicate encore l'éviJuation de la portée moyenne da fail do l'ctalement a la voine guthils provoruent, el qui rendent souvent impossible le calcul de la porter theorigue faute de comnaitre lincidenee rélle du jet at dépirl (planche $Y$ );

- 1)ifficulte due à la dissipation dénergie dans lair qui, pour des lames minces ou bien divisées, réduit la portée, et conduit à une évaluation par défaut de la vilesse initiale;

...- Enfin, conformément à ce qui a été exposé plus haut, l'́valualion de la perte de charge relative, somvent faile ì dibit moyen ou fable, ne permet pas de prejugex de la valeur que prendra eetle perte le charge au délit maxima.

Par contre, sur molik reduil, celle molhode mous parait tres valable, lobscrvalion chant facifiter clu fat de l'absence d'amulsion. En outre. la dissipation d'énergie da jel dans l'ail peut ibe megligere pour des jels compants yuil ext loujours possible de reabiser en supprimant le dispositif de dispersion. I.es mesures realisées sur le modele de Bont pour deux debits onl donne des resultals qui roüncident asse\% bien avec les valems de la perte de chatge déduites de Ia mesure du lirant d'eau à la sortic du coursier.

Pal exemple, all debil maximum, dans fe (als du radier en ciment lissé, on a obtenu $\left(\Delta \mathrm{H} / h_{0}\right)=37 \%$ contre $33 \%$ par la premiere méthode.

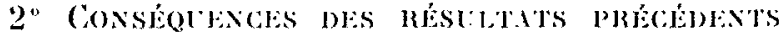 SUR LA THELE DES ONYRAGES IOT LA DISSIPATION DI: L'B́NERGIL:}

Nous envisagerons sucessivement les conclusions que l'on pent lirer de ce qui précede, jour les ouvrages eux-mèmes et pour la dissipalion de l'énergie à l'aval.

\section{(1) Elforts imposcis allx onverges:}

Ces eliorls somt la cause de la jerte de charge sur le coursier, el lon smppose que la folalile de. la furbulence cxistanl dans la reine a la sortic provient du frottement du radier. Les eflorts sur le radier varient done comme l'énergie dissipie sur l'ouvlage, si l'on suppose négligeable la variation de la vilesse moyenne en fonclion du dehit; celle approximation est valable en dehors du domaine des tres pelils débits. On diduit dome aisément du diałgramme de la disure 2l l'allure du diagramme divolution des efrorts de froflement imposes a lourrage en fonclion da debil (liga. 3t).

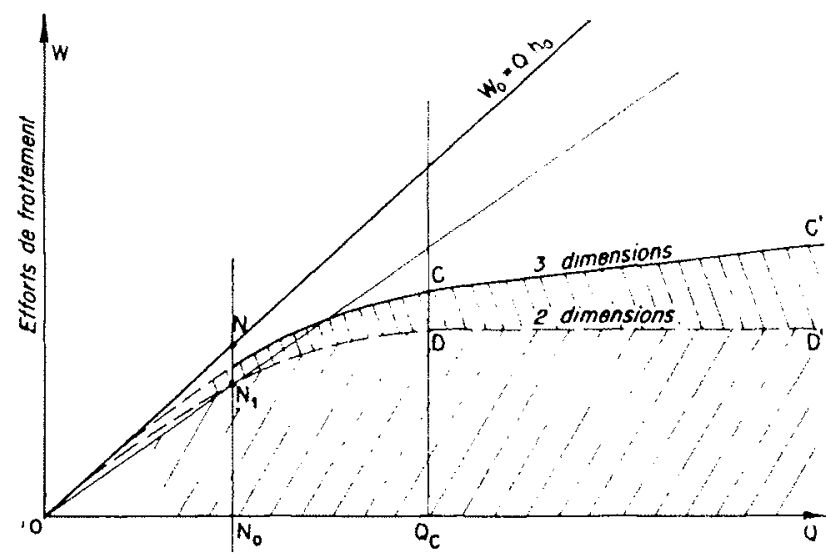

Fon. 3t. - Evolution des efforls da froflement sur be coursier dien ciacualeur

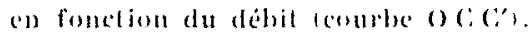

On roit que les alforts croissent jusquatu dehil crilicue pour n'angmenter que tres peu ensuile. C'est co qui a che verifie lors des mestures def-

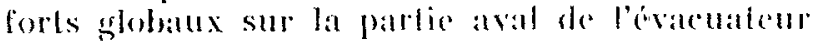

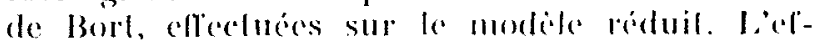
forl tansentive dù al froltrment ne variail pratiquement pas entere les kibits de bog) $130 / \mathrm{s}$ al

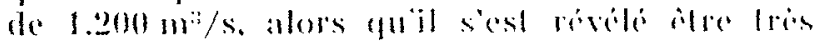
semsible, commone prove, atus varialions de mugusili.

D'aulre part, les mesures failes monlrent que

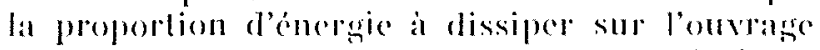

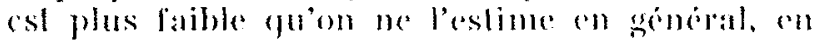

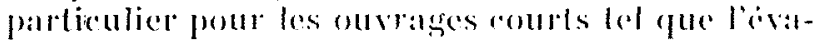
cuateur de Chastang.

On voit done que levalualion des eflorts inposes par l'iemlement anx ouvages a certajement loujours ble falle avee wne loies large sedurite, dautant plus que si on chudie la repartition générale des efforts tangentiels el nomanax appliqués à l'ouvrage, en partant des répartitions do pressions, on constate gue la compesante horizontale des aforls globaux appliques a l'ouvarue est toujours dirigce vers l'amont. Dans le cas de l'ouvrage de Bort par exemple, la composanto horizontale des efforts de pression dirige vers lamont est de 3.000 tonnes onviron, alors guo la composante des efforts de frobtement dirisge. 


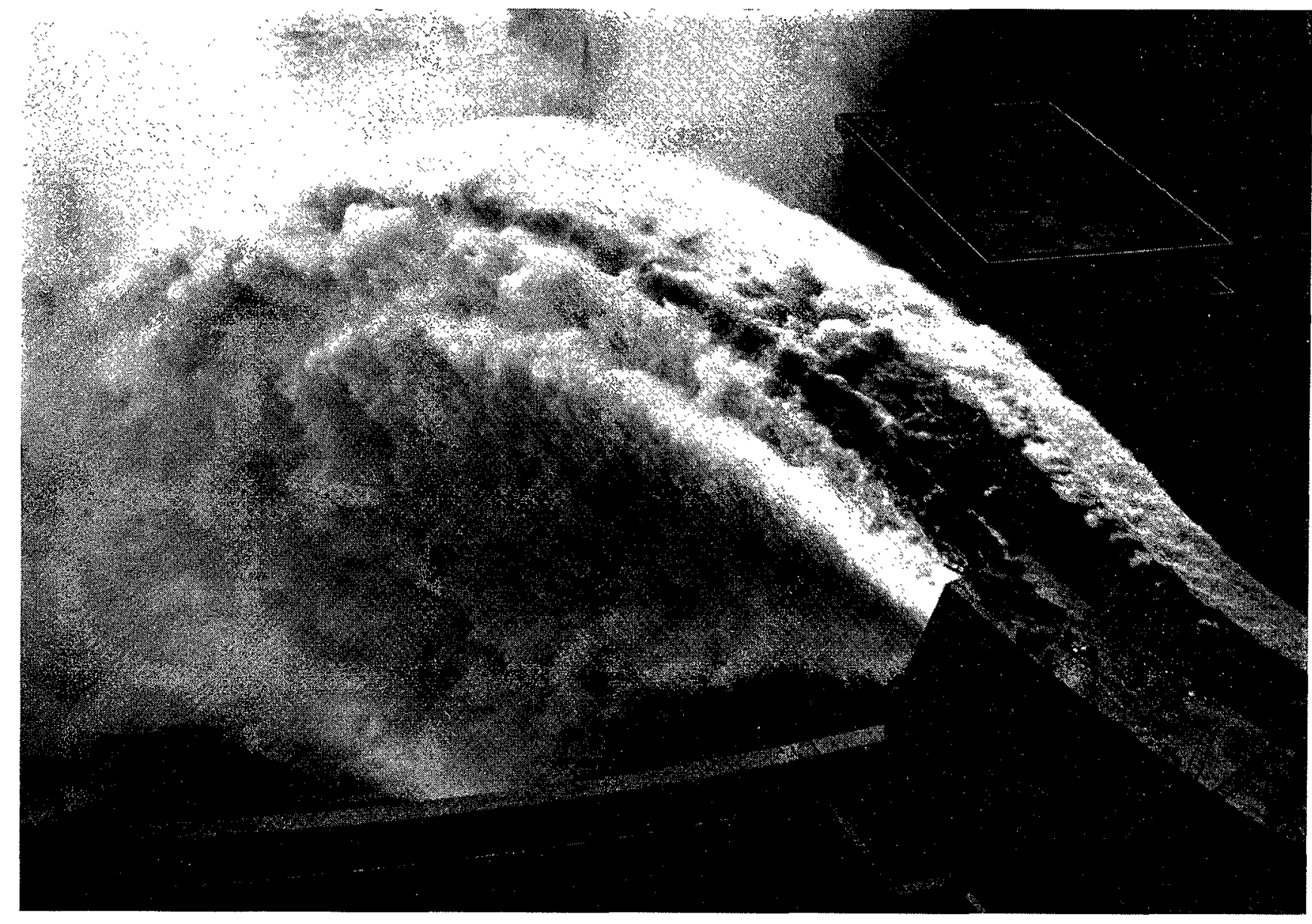

Fig. 35. - Chastang $\left(Q=600 \mathrm{~m}^{3} / \mathrm{s}\right)$.

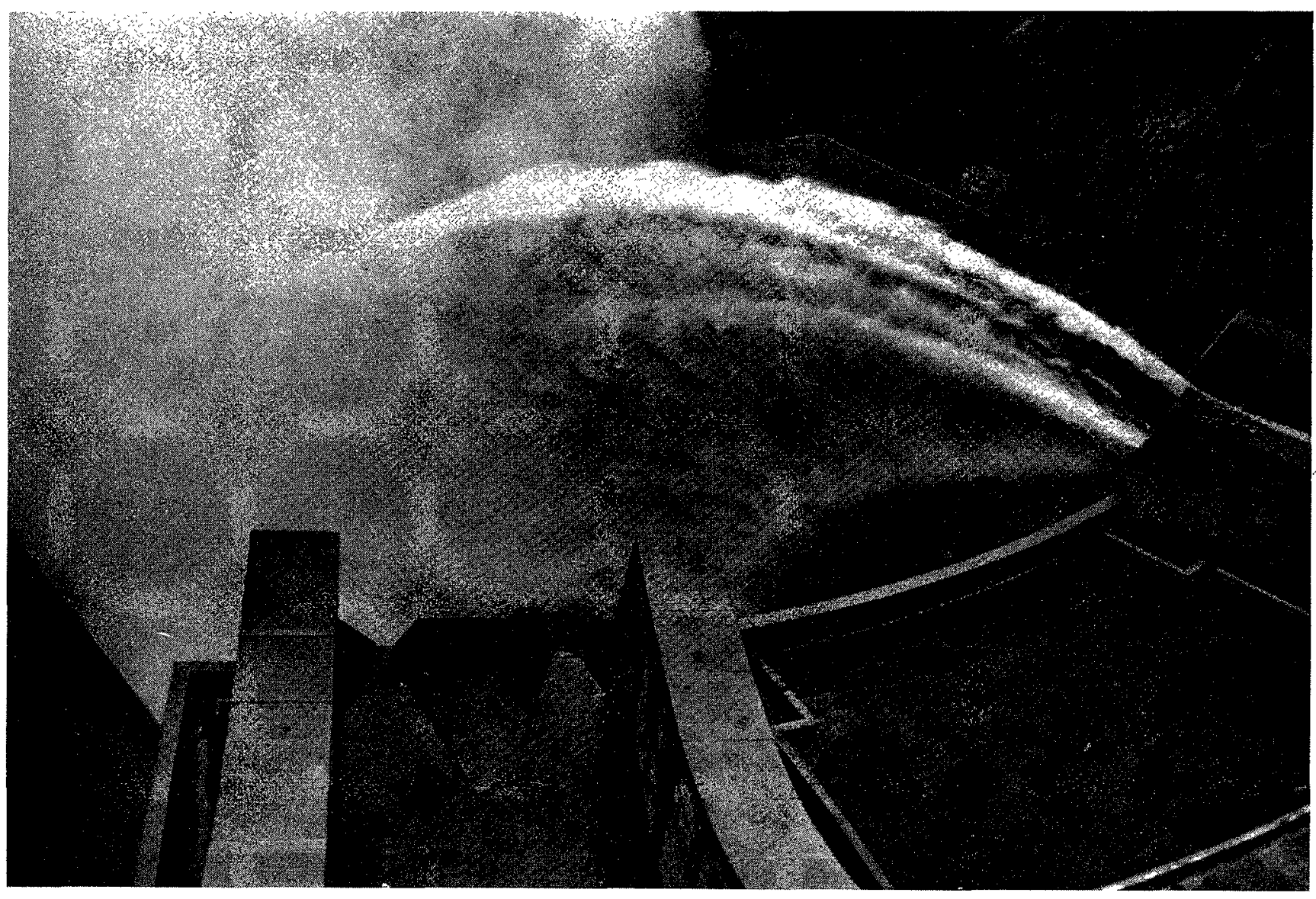

Fig. 36. - Chastang $\left(Q=600 \mathrm{~m}^{2} / \mathrm{s}\right)$. 


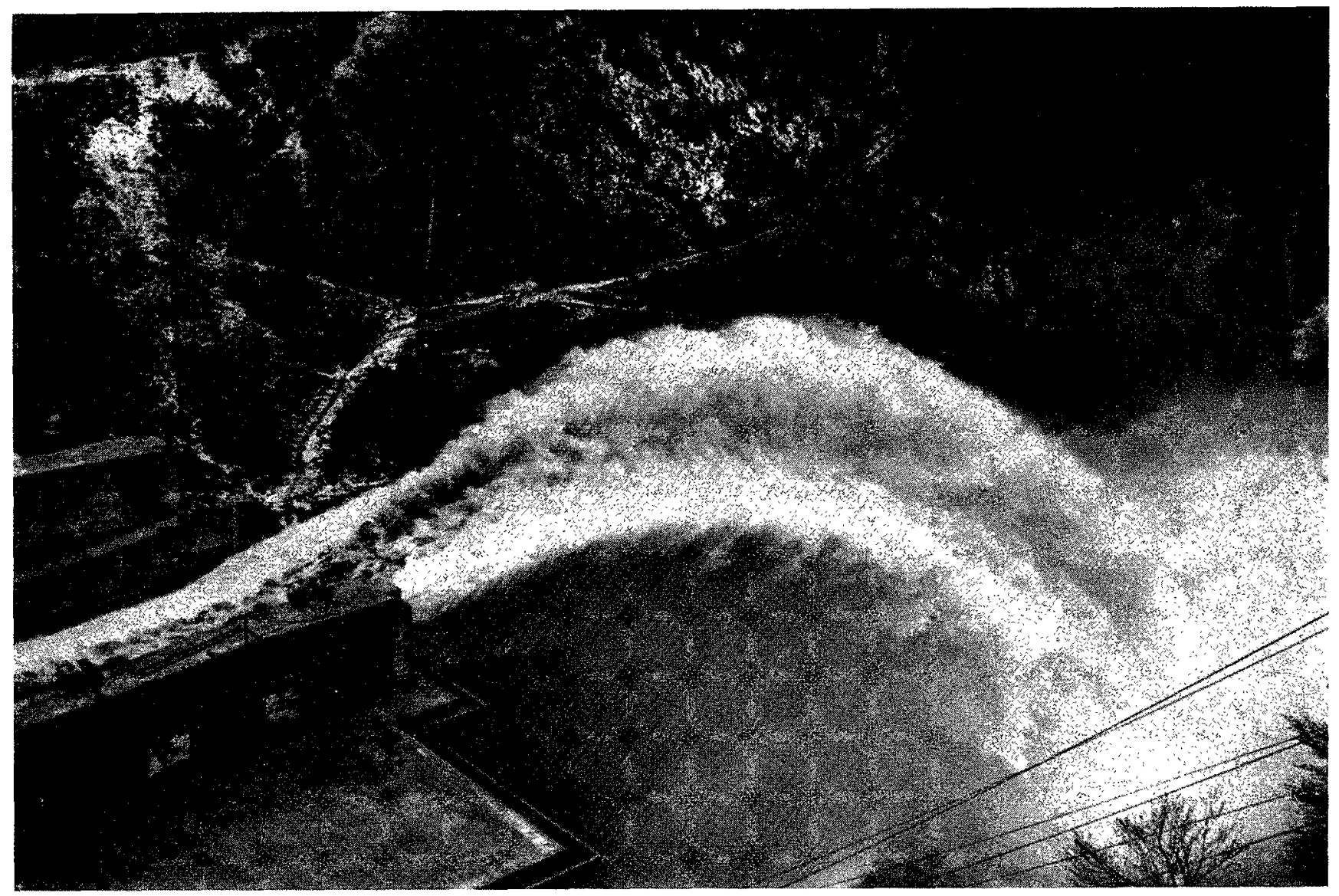

Fig. 37. - Saint-Emienne-Cantalès $\left(Q=230 \mathrm{~m}^{3} / \mathrm{s}\right)$.

vers l'aval est comprise entre 900 et 1.300 tonnes. Les efforts de frottement n'intervienment donc que si la partie aval de l'ouvrage n'est pas solidaire de sa partie amont, ce qui se produil parfois pour les évacuateurs en saut de slii, lorsque Jeur partie aval repose sur le toit de l'usine.

\section{b) Dissipation de l'énergie à l'aval :}

Nous considérerons particulièrement ici le cas des évacuateurs en saut de sli, puisque tous les exemples cités ci-dessus sont pris dans cette calégorie d'ouvrages.

Les mesures réalisées à Saint-Etienne-Cantalès ct à Chastang pour des débils inférieurs à la moilić du débit maximum montrent que l'énergie dissipéc sur l'ouvrage atteint seulement $19 \%$ et $12 \%$ de l'énergic totale respectivement; cetle faible proportion diminuerait encore pour des débits plus importants. Ceci est la conséquence de l'évolution progressive ver's des évacuateurs de surface de capacité de débit accrue et donc relativement beaucoup plus courts.

Il n'est pas certain que l'on ait prêté toujours suffisamment attention au probleme de la dissipation de cette quantité énorme d'énergie contenue dans la veine à la sortie de l'ouvrage. Celte ćnergie se dissipe sous deux formes:

- Emulsion et frottement dans l'air;
- Tourbillons dans la f'osse aval et érosion des parois de celle-ci.

Si on raisonne par analogic, il semble vraisemblable que l'importance relative de la dissipation d'énergie par frottement dans l'air diminue aux forts débits, comme c'est le cas pour le froltement sur l'ouvrage.

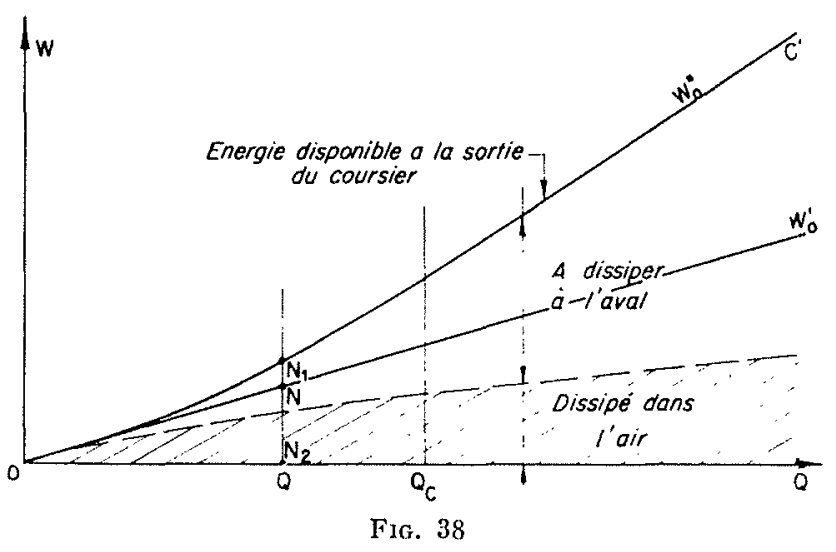

On a reporlé, à tilre indicalif, sur le diatgramme de la figure 38 , la courbe déduite de la figure 21 et représentant la valeur de l'énergie totale disponible à la sortie du coursier :

$$
W^{\prime \prime}{ }_{0}=W_{1}+W_{0}^{\prime}
$$




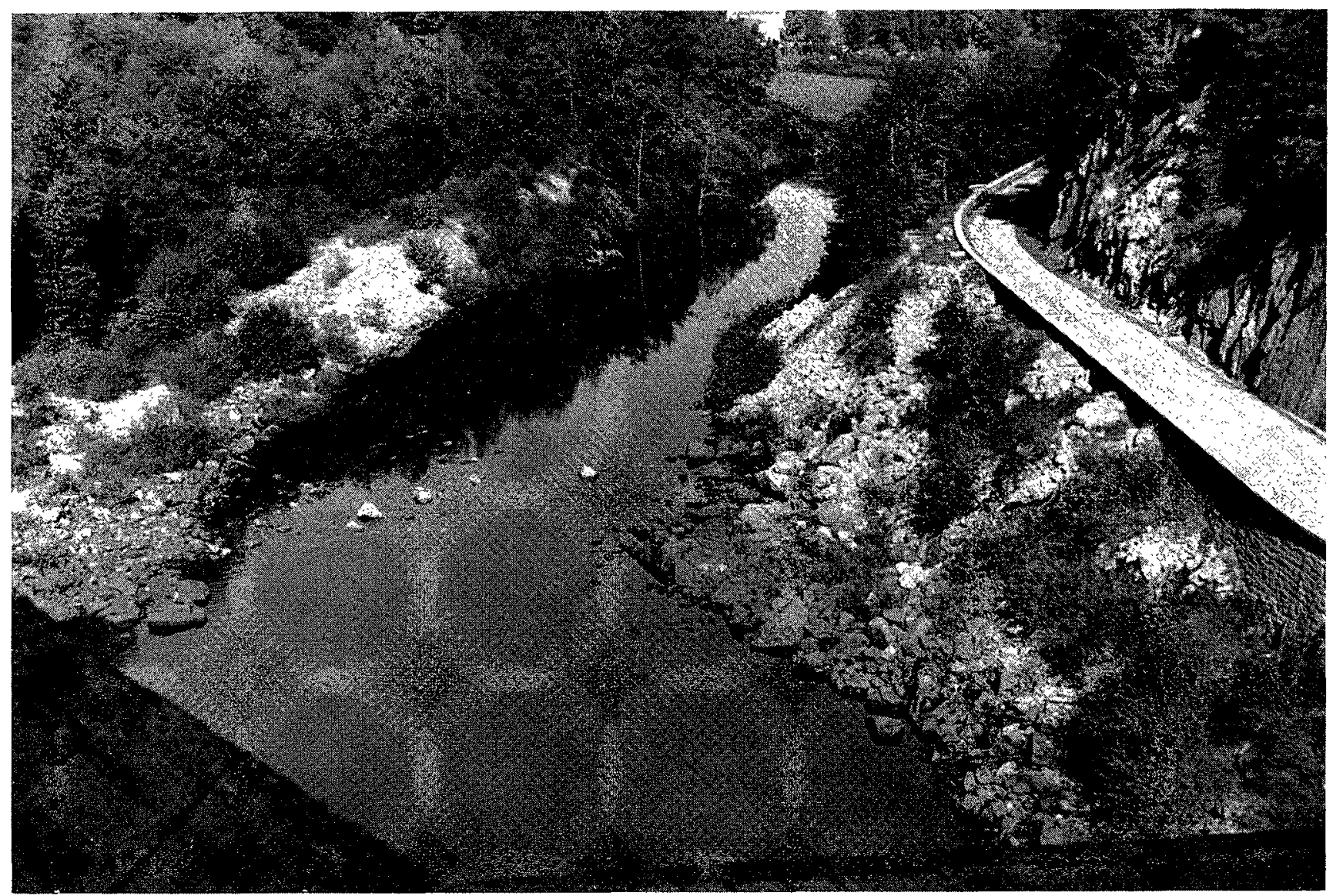

Fig. 39. - Etat des rives avant le déversement.

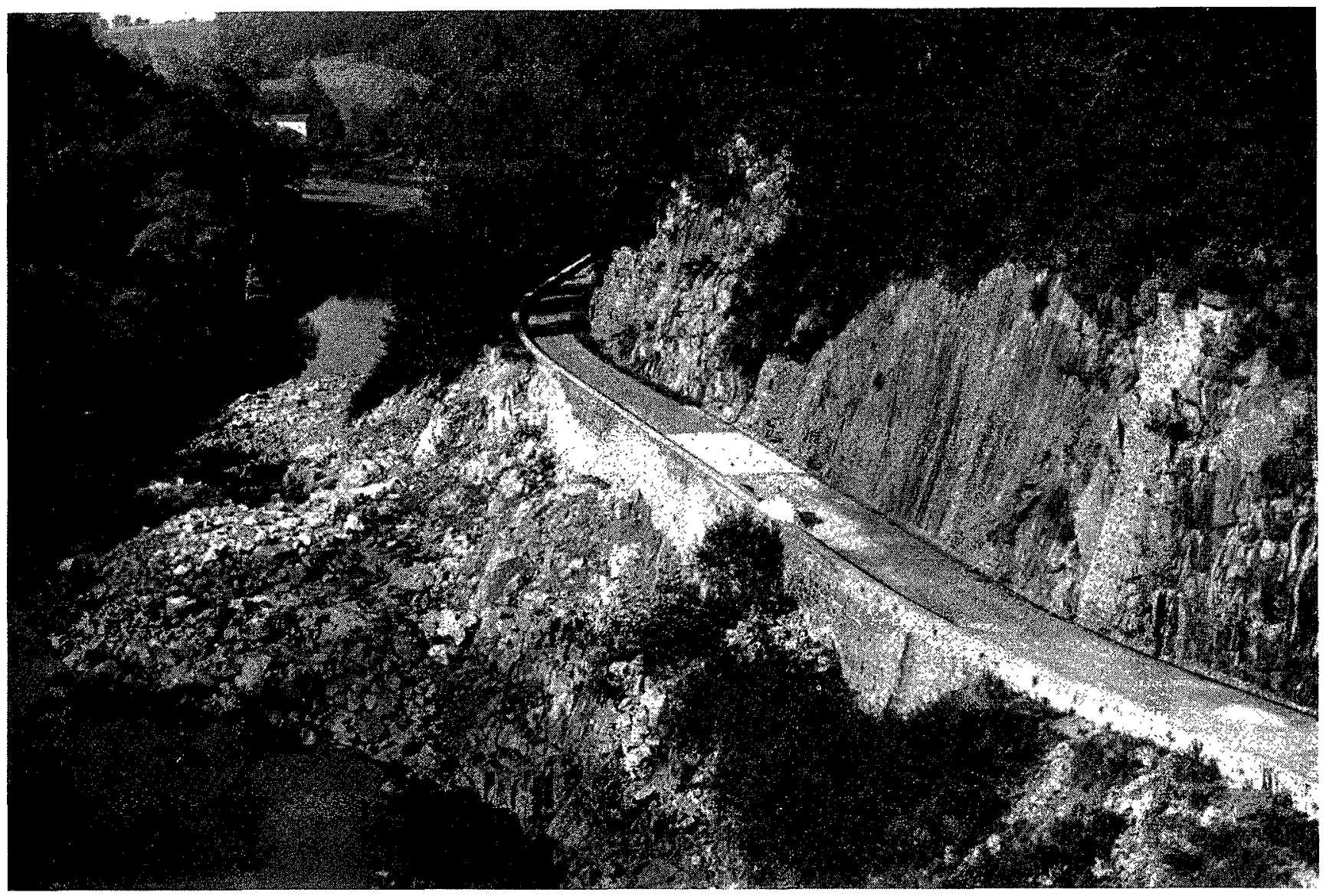

Fis. 40. - La rive droite après le déversement á $450 \mathrm{~m}^{3} / \mathrm{s}$ (réparations effectuées). 


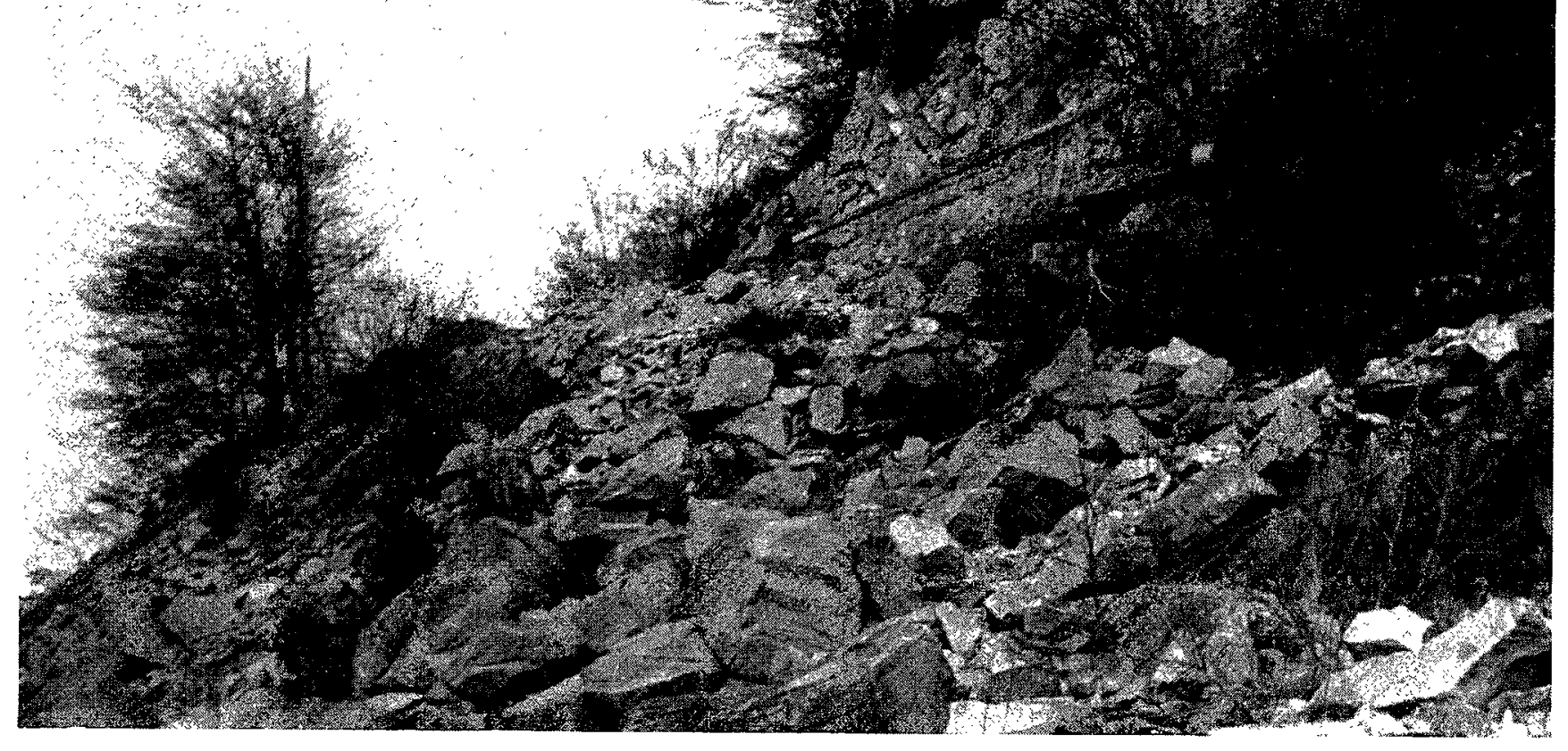

(3)

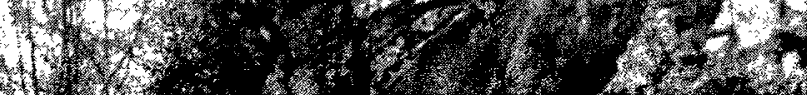

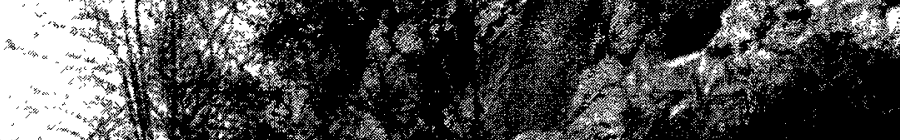

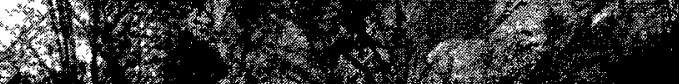

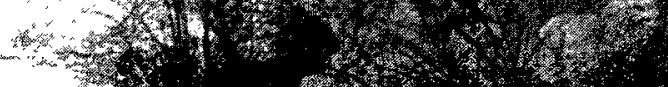


et on a supposé que la variation de l'énergie dissipée dans l'air pouvait être représentée par une courbe analogue it celle représentent l'énergie dissipée sur l'ouvrage.

$\mathrm{Si}$ ces hypotheses sont exactes, on voit que l'énergie à dissiper à l'aval par tourbillons ou érosion du fond croît comme une puissance du débit trés supérieure à l'unité.

Cer qui précède souligne l'imporlance de l'étude du rispositif de dispersion de la veine d'une part, de la réception du jet à l'aval d'atutre part, études qui ne peurent guère se faire autrement que sur modile réduit.

Il nous est diflicile d'aller plus loin, le problime de conception des ouvages etant un problime economique autant que lechnique. T'oulefois, on pent se demander si dans certains cas vì le problime aval est particulierement délicat, l'augmentation volontaire de la longueur relalive des cracmateurs de surface. ou même de leur rugosité, ne permettrait pas d'en laciliter la solution. Il faut noter que pour des ouvrages relalivement longs où le debit eritique serait ćlevé, il se poserait pour tous les débits inferieurs à ce débit critique la question du gonflement de la veine par émulsion superficielle.

Enfin, nous pensons utile de preiciser que dans les cas où l'on compte sur le jet lui-méme pour creuser sa fosse d'amortissement jusqu'à un état d'equilibre, il convient de s'assurer sur modile quancune des étapes intermédiaires ne constituera un danger pour les ouvrages, comme cela a été le cas pour les évacuateurs de St-Etienne-Can- talès. Un déversement de $2 \times 230 \mathrm{~m}^{3} / \mathrm{s}=460 \mathrm{~m}^{3} / \mathrm{s}$ pendant quelques dizaines de minutes a en eflet donné lieu à une attaque notable du talus Rive droite (photos PI. VI) et a des projections tres importantes de fusées d'eau et d'embruns sur la falitise dominant la route; le ruissellement considerable qui en est resulté sur cette falaise a inondé la route et aurait pu inonder la Centrale s'il ne s'était pas agi d'un essai dont on pouvait limiter la durée. Il est à noter que le fonctionnement du seul évacuateur Rive droite a même débit pendant une heure, lors des essais relatés plus haut, n'avait donné lieu à aucun incident.

L'explication de cettc anomalie apparente nous paraît être la suivante : le jet à Saint-EtienneCiantalès n'est pratiquement pas disperse, mais seulement rejeté au loin par un tremplin situe a l'aval des évacuateurs; la surfice d'impacl de chacune des veines est donc trìs réduite. D'autre part, le matelas d'cau n'est pas tres important du fait de la faible profondeur actuelle de la fosse naturelle. Lorsque le pertuis Rive droite fonctionne seul, le jet qui s'écrase sur le fond du chenal peut s'étaler lateralement vers l'emplacement laissé libre sur sa gauche. Par contre, dans le cas $d u$ fonctionnement simultané des deux passes déversantes, le jet Rive droite se trouvant bloqué entre le jet voisin et la rive, ne dispose pius d'un volume d'expansion suffisant, si bien qu'il se produit un « rebondissement » de paquets d'eau constituant unc fraction tres faibie du débit, mais suffisante néanmoins pour provoquer des incidents qu'il serait préférable d'éviter.

\section{CONCLUSIONS}

La mise au point d'une méthode optique de relevi d'une surfice liquide a rendu possible la réalisation de mesures assez précises sur des ouvrages réels. Les résullats de ces essais confrontés avec ceux obtenus soit sur modèle réduit, soil par voie théorique, ont permis :

De montrer que les écoulements à grande vilesse obéissent aux lois classiques de lhydraulique, et que la similitude de Frotus reste valable, sauf en ce qui concerne lémulsion, pour ces écoulements a tries grand nombre de Rexoloss;

De preciser l'évolution relative des différenles formes de dissipation d'energie en fonetion du débit;

De montrer que la proportion d'énergie dis- sipée sur les ouvrages est inférieure à l'estimation généralement admise et que, par suile, l'essentiel de celte dissipation d'énergie a lieu à l'aval des ouvages.

'Toutefois, il reste des problèmes que nous n'avons pu aborder lels que la répartition de la dissipation d'énergic entre le frottement des jets dans latir et l'érosion et les remous à l'aval, faute do moyens do mesure. Il en est de même de la recherche des Jois du développement de la couche émulsionne existant aux petits débits, problime qui prendrait une importance accue dans le cas où on serail amené à s'orienter, pour les évacuateurs de surface, vers des ouvages de longueur relalive elevéc afin de diminuer la proporlion d'énergie restant à dissiper à l'aval. 
M. le Président Hupen félicile MM. Marrne et Obol.kNSKY de leur étude intéressante. Il pense, au point de vue terminologie, que le mot « vame segment $»$ convient micux que « vanne secteur », pour désigner les vannes en service à Saint-Etienne ou à Chastang, lesquelles interviennent par leur segment et non par le plan du dièdre. Puis, il demande s'il a été fait d'autres essais comme ceux-ci en grandeur naturelle.

M. Matrme signale les essais de l'évacuateur de l'Aigle réalisés il $y$ a quelques années sur l'initialive de II. Covve, qui en a relaté les résultats dans sa communication au $I I^{*}$ Congrès des Grands Barrages : les expérimentateurs ne disposant pas d'appareillage spécial, le relevé des tirants d'eau avait été effectué le long d'un gui. deau, au moyen d'un fil à plomb. Ce moyen de mesure ne permet pas, compte tenu de la présence de la couche latérale émulsiomnée, d'obtenir une précision supérieure à $10 \%$, et le gonflement dù à l'émulsion donne lieu de penser que cette erreur est très probablement une erreur par excés, et, d'une erreur relative de $10 \%$ par excès sur le tirant d'eau résulte une erreur de $20 \%$ par défaut sur la charge au point considéré. Or, le résultat de ces mesures laisail apparaitre à l'aval du coursier une charge réduite à la moitié de sa valeur initiale; la valeur corrigée de cette perte de charge serait donc de $4.0 \%$ de la charge totale au-dessus du point bas du cour sier, ce qui differe très peu de la valeur de $35 \%$ trouvée à Saint-Etienne-Cantalès pour un tirant d'eau relatif $d / \mathrm{L}$ légèrement plus fort.

M. Bovmavision indique l'essai d'un ingénieur américain, utilisant une poutre avec des touches pour déterminer le plan d'eau; cet essai peu réussi n'a pas été publie.

Sur la demande de M. le Président, M. Martne précise que la vanne etait ouverte partiellement au Chastang, mais totalement à Saint-Etienne où, toutefois, le plan d'eau avait été baissé pour éviter un trop grand débit. En conséquence, la perte de charge mesurée au Chastang comprenait forcement, outre le frottement sur le coursier, la perte singulière à la traversée de la vanne que l'on peut, toulefois, considérer comme négligeable si l'ouvrage est bien tracé el l'écoulement alimenté par un plan d'eau calme. En tout état de cause, si l'on doit retrancher de la perte de charge mesurée à Chastang, la perte de charge singulière due a la vanne, les conclusions de ees essais, montrant les faiblesses des pertes de charge sur te coursier aux forts débits, s'en trouvent renforces.

Sur une question de M. le Président, relative à la certitude d'avoir atteint les vitesses limites malgré le débit particl, imposé par les cireonstances, M. Maitre répond que la vitesse est theoriquement constante it la surface de l'éconlement, quel que soit le débit, puisqu'elle dépend uniquement de la hateur de chute sur l'ouvrage, a condition, loutefois, que le débit soit supérieur au débit critique, c'est-à-dire que l'écouloment en surface soit à potentiel; cette condition ctait remplie a Chastang, les essais ayant ćté réalisés pour un débit double du débit eritique. Les vilesses mesurées à Chaslang par M. OвoreNsky s'établissaient d'ailleurs entre 27 et $27,90 \mathrm{~m} / \mathrm{s}$ svirant les sections, et encadraient la vitesse prévue par la loi de Bernoulli à 5 ou $10 \%$ près.

M. IlaEgendes s'intéressant a l'effort sur le cousier M. Marrne indique que l'effort dirigé vers l'aval, dû à la traction de lécoulement sur l'ouvrage, est exprimé globa- lement, suivant le théorène des quantités de mourement. par $\varrho Q\left(V_{0}-V\right)$; $V$ étant la vitesse réelle à l'aval de louvrage, et $V_{0}$ la vitesse théorique en l'absence de frottement. Toutefois, la répartition de cet effort $F_{0}$ le lons du coursier est difficile a étudier, la précision des mesures n'étant pas suffisante pour calculer exactement la variation de quantite de mouvement - ou la perte de charge - entre deux sections consécutives.

M. Marte pense qu'on a, d'ailleurs, lendance a exagérer l'importance de cet eftort, en ce qui concerne l'équilibre global de l'ourrage, car il est largement équilibré par la résultante des efforts de pression de l'écoulement sur le coursier, resultante dont la compusante horizontale $F_{2}$ est dirigée vers lamont; on at évalué ces deux efforts pour l'évacuateur de Bort à partir des mesures de pression et de ligne d'eau réalisées sur le modele : l'ordre de grandeur de $F_{2}$ est de 3.000 connes, contre 1.000 tonnes seulement pour $F_{1}$. Pat contre, Iorsque lon considère l'équilibre de la partie aval du coursier, non solidaire du barrage et reposant sur" le toit de l'usine, il faut tenir comple de l'effort de traction vers l'aval dû au frottement, car la résultante des forces de pression est pratiquement verticale dans cette partie de louvrage et n'intervient donc plus. Pour l'usine de Bort, on a mesuré directement sur modèle réduit leftort global de traction vers laval résultant du frottement. Ces résultats ont confirmé, dans ce cas, l'evaluation approchée obtenue à partir de l'évaluation de leffort a la paroi $\tau_{0}$ résultant de la formule de Mansing-Strnoksen. Il est à noter que celte concordance est probablement due à ce que l'écoulement est très peu diff'erent d'un écoulement pleinement turbulent a l'aval de l'évacuateur de Bort, du fait de sa très grande longueur relative. Il n'en serait pas nécessairement de mème pour un ouvage plus court, sur lequel on peut penser que les efforts seraient plus faibles.

M. Laxglots remarque que l'énergie dissipée a l'aval surpasse, dapres la communication de M. Matrue, les évaluations faites dans le passé. Tne crue récente de la Dordogne ayant cause des degats a laval de Chastang, pose aux Services de l'Exploitation d'li.D.F. le probleme de l'évaluation exacte de l'énergie restant a dissiper a la base du jet.

M. Martne pense que c'est bien là que réside le problème essentiel, mais que les mesures, en ce domaine, sont pratiquement impossibles, à lheure actuelle. En ce qui concerne Chastang, M. Martne pense que les dégâts causés par la récente crue de $1.700 \mathrm{~m}^{3} / \mathrm{s}$ ont surtout mis en évidence l'importance de phénoménes secondaures:

- Ces dégàts sont en effet localisés hor's de la zone d'impact proprement dite qui, bien protégée, a convenablement rèsisté;

- D'autre part, ils sont dus surtout à l'importance de la crue dans un chenal non calibré pour un tel debit et à l'attaque des berges, peut-ĉtre insuffisamment prolégées, par l'agitation de surface qui se propage vers l'aval à partir de la zone d'impact, à la manière d'une honle irrégulière et violente.

Sans insister spécialement sur te cas particulier de Chastang, cité à titre d'exemple, M. Laveloors pense, en lout cas, que tenant compte des remarques de M. Martre, il y aurait sans doute lieu, dans l'avenir, d'apporter une plus grande prudence dans l'évaluation de l'énergie à dissiper a l'aval de ec genre d'ouvrage. 